\title{
Connecting West and East
}

Citation for published version (APA):

Zhang, M. (2019). Connecting West and East. [Doctoral Thesis, Maastricht University]. Gildeprint Drukkerijen. https://doi.org/10.26481/dis.20190701mz

Document status and date:

Published: 01/01/2019

DOI:

10.26481/dis.20190701mz

Document Version:

Publisher's PDF, also known as Version of record

\section{Please check the document version of this publication:}

- A submitted manuscript is the version of the article upon submission and before peer-review. There can be important differences between the submitted version and the official published version of record.

People interested in the research are advised to contact the author for the final version of the publication, or visit the DOI to the publisher's website.

- The final author version and the galley proof are versions of the publication after peer review.

- The final published version features the final layout of the paper including the volume, issue and page numbers.

Link to publication

\footnotetext{
General rights rights.

- You may freely distribute the URL identifying the publication in the public portal. please follow below link for the End User Agreement:

www.umlib.nl/taverne-license

Take down policy

If you believe that this document breaches copyright please contact us at:

repository@maastrichtuniversity.nl

providing details and we will investigate your claim.
}

Copyright and moral rights for the publications made accessible in the public portal are retained by the authors and/or other copyright owners and it is a condition of accessing publications that users recognise and abide by the legal requirements associated with these

- Users may download and print one copy of any publication from the public portal for the purpose of private study or research.

- You may not further distribute the material or use it for any profit-making activity or commercial gain

If the publication is distributed under the terms of Article $25 \mathrm{fa}$ of the Dutch Copyright Act, indicated by the "Taverne" license above, 


\section{Connecting West and East}

Ming Zhang

张明 
(C) Ming Zhang, 2019

Connecting West and East

Thesis, Maastricht University, Maastricht, the Netherlands

Layout: $\quad$ Ming Zhang

Cover design: Guido Haenen

Printed by: Gildeprint, Enschede

ISBN: $\quad 978-94-6323-723-9$

All rights are reserved. For articles published, the copyright has been transferred to the respective publisher. No parts of this thesis may be reproduced, stored in a retrieval system or transmitted in any form or by any means, without prior permission from the author. 


\title{
Connecting West and East
}

\section{DISSERTATION}

\author{
To obtain the degree of doctor at Maastricht University \\ on the authority of Rector Magnificus, Prof. Dr. Rianne M. Letschert \\ in accordance with the decision of the Board of Deans to be defended in public \\ on Monday, the July 1 st at $12.00 \mathrm{hrs}$
}

by

Ming Zhang

张明

born on 23 December 1988

in Zheng Zhou, China 


\section{Promotor}

Prof. dr. Guido R.M.M. Haenen

\section{Co-promotor}

Dr. Mohamed Moalin

\section{Beoordelingscommissie}

Prof. dr. Harry Steinbusch (Voorzitter)

Dr. Gino van Strijdonck (Zuyd University of Applied Science, Geleen)

Prof. dr. Harry Struijker Boudier

Prof. dr. Ron Heeren

Prof. dr. Zhi-Qing David Xu (Capital Medical University, Beijing, China)

The research described in this thesis was conducted at Department of Pharmacology and Toxicology, NUTRIM School of Nutrition and Translational Research in Metabolism of Maastricht University. The work in this thesis was financially supported by China Scholarship Council (CSC, File No. 201508460007). 
To my parents and family

献给我的父母和家人

Amor Vincit Omnia

大爱无疆 



\section{Contents}

$\begin{array}{ll}\text { Preface } & 9\end{array}$

Chapter 1 Connecting Western and Eastern Medicine from an energy perspective

Chapter 2 The cardiovascular side effects of Ma Huang due to its use in isolation in the Western world

Chapter 3 The screening of anticholinergic accumulation by Traditional Chinese Medicine

Chapter 4 The chemical reactivity of (-)-epicatechin quinone mainly resides in its B-ring

Chapter 5 Unravelling the redox modulating activity of quercetin: a systematic approach

Chapter 6 General discussion and summary

Chapter 7 Nederlandse samenvatting en algemene discussie

Valorisatie addendum

Chinese translation

Acknowledgment

Curriculum Vitae

List of publications 



\section{Preface}

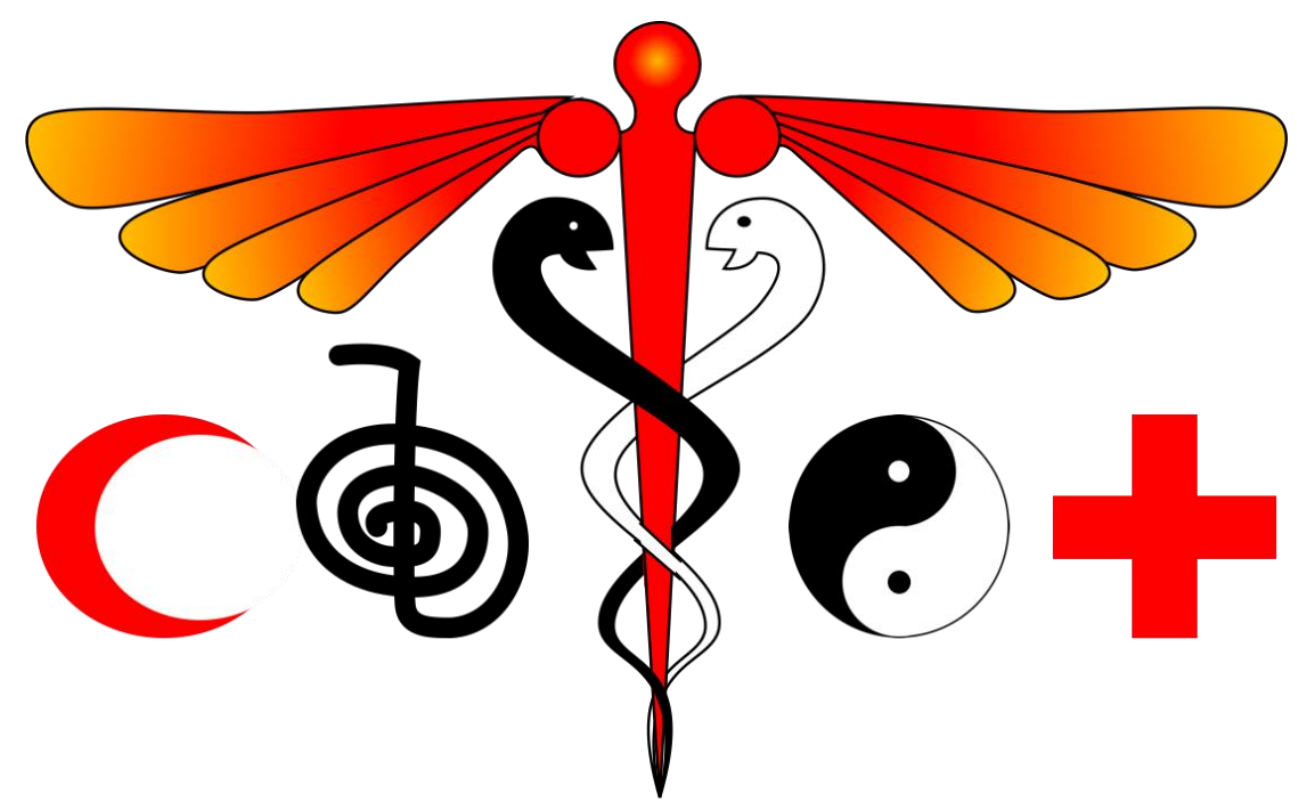


The number one desire of most people is to be in good health. This is also reflected by the high social status of medical professionals in all societies. In most cultures, the medicine woman/man has her/his own "uniform", uses a jargon that is not easily understood by others, and specific symbols are used (Fig. 1).
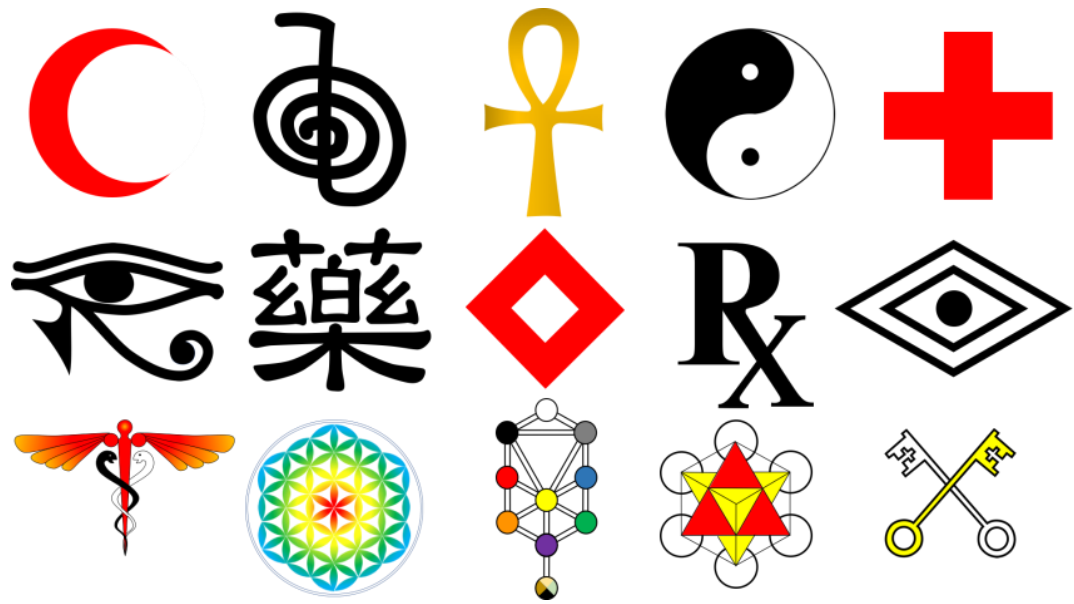

Fig. 1. Symbols of Health, Medicine and Life in several cultures.

Despite these similarities, medicine has been developed differently in various cultures. For example, Western medicine is quite different compared to Eastern medicine. They diverged because they are built on different fundaments. Both have their merits as well as limitations. The general idea that now arises is that both types of medicine are complementary and that we could benefit by connecting Western and Eastern medicine to get the best of both worlds [1,2]. This is in line with the "Global Strategy and Plan of Action on Public Health, Innovation and Intellectual Property" adopted at the 61st World Health Assembly in 2008, which states that traditional medicine should be further developed by stimulation of research and innovation [3]. This prompted us to further unravel the molecular mechanism of the mode of action and toxicity of Traditional Chinese Medicine (TCM) to try to connect TCM with Western medicine.

\section{The fundament of Western medicine}

Western medicine is "evidence based", which means that all treatments are rational: from the molecular level to the final therapeutic effect on patients. It evolved from the use of herbs, to the isolation and use of the most active ingredient in herbs, to the use of (semi-) synthetic compounds [4,5]. Relatively recent acquired knowledge on the chemical structure of drugs and on specific targets, e.g. receptors, made it possible to elucidate the molecular mode of action (Fig. 2) [6,7]. This has revolutionized Western medicine. One of the biggest achievements of Western medicine was the development of antibiotics, that have saved the lives of millions of people. The goal in drug development is to increase activity and specificity, and to design drugs that have a strong, specific and immediate effect. The effect of drugs is tested in clinical trials, performed according to very strict guidelines. This 
finally results in evidence based, standardized guidelines for diagnosis and treatment of diseases $[8,9]$.
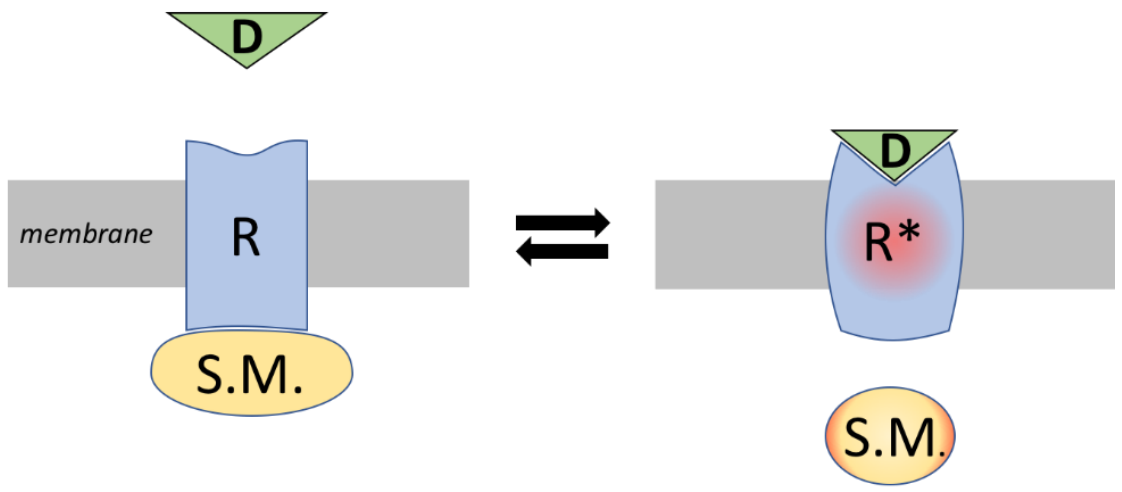

Fig. 2. The drug-receptor concept in Western science. The paradigm is "Corpora non agunt nisi fixata", a drug (D) will not work unless it is bound to a specific target, e.g. a receptor (R). Binding of the drug to the receptor will cause a conformational change of the structure of the receptor $\left(\mathrm{R}^{*}\right)$. This triggers the production of a second messenger (S.M.), which will finally result in a strong effect in the cell.

\section{The fundament of Eastern medicine}

Eastern medicine has an intuitive fundament. TCM has been developed in China over centuries and it is still practiced according to this old tradition [10]. Eastern medicine has been one of the driving forces of the rise of the Chinese culture. In TCM, herbs are combined in a specific proportion. The concept is that herbs work together to get the best response. There are no specific targets. In general, the effect of TCM is relatively soft and it takes a long time to cure a disease. However, the side effects of TCM are low [11,12]. In TCM, patients are treated in a "personalized" way according to the tradition and to the doctor's experience [13].

\section{The limitations of Western medicine}

From a Western point of view, there is no doubt that the "scientific" Western approach is far more superior to the Eastern "intuitive" approach. However, numerous examples indicate that in the West a more humble attitude is more suitable. One of them is the unexplained effect of paracetamol. Although paracetamol is the most used drug and is applied over several decades, its molecular mode of action is not elucidated [14]. This indicates that the mysterious "placebo" effect contributes to the therapeutic effect of paracetamol. Another aspect is that many Western drugs fail to be effective on the long run. Compared to TCM, Western drugs have a very short half-life [15,16]. Even antibiotics tend to lose their effectiveness, as microorganisms adapt by becoming resistant. Moreover, the effects of Western drugs are often not as specific as proclaimed, and they do not act on the single receptor aimed for. For example, many drugs display some anticholinergic activity as side effect. When several of these drugs are given to a patient, this might give rise to "anticholinergic accumulation" [11,17]. In addition, critical examination indicates that many controlled clinical trials, the fundament of evidence based medicine, actually may 
give false results $[7,18]$. It is more realized that most scientific findings, even if they appear to be absolutely true, could be incorrect [19]. At the best Western science is based on axioms, but often this is only on paradigms, presumptions, prejudice, or ultimately hubris. Moreover, most of the pharmaceutical industries aim to generate as much money as possible, and their primary interest is not the welfare of the patient. Fraud and manipulation of scientists and doctors are not uncommon in Western medicine. Pharma industry is even accused of inventing and spreading diseases with the single goal to market their "miracle cure" (e.g. ritalin) [20,21].

\section{The limitations of Eastern medicine}

The molecular mechanism of TCM is lacking. For example, there is no scientific prove for the existence of meridians [22]. This means that in the West, the fundaments of TCM are denied and East and West are not connected. Nevertheless, it is increasingly realized that also in Western societies TCM is valuable for treating diseases, especially when the synthetic designed drugs fail to be effective. One of the problem is that the use of TCM has led to adverse effects in the West [5,23]. For example, Ma Huang is a herb that has been used over centuries in China to treat asthma. By combing it with other herbs and using a relative low dose in TCM, the side effects caused by Ma Huang are reduced to a relative safe level. However, in the US, Ma Huang was used without the combination with other herbs, and in a relative high dose with the aim to lose weight or to act as a stimulant. This improper use of Ma Huang gave rise to serious cardiovascular problems (Fig. 3) [24-26]. This is in line with the Western strategy to aim for a strong and quick effect, and has led to the improper use of TCM which causes the side effects. This has given TCM a bad reputation. This hampers the correct appreciation of TCM. To give TCM a "scientific" basis and to elucidate its molecular mechanism, Western science needs to study TCM in the way it is supposed to be used. Moreover, Western scientists should be more open minded to the concepts of Eastern medicine and accept that the West can learn from the East. 


\section{Traditional Chinese Medicine}

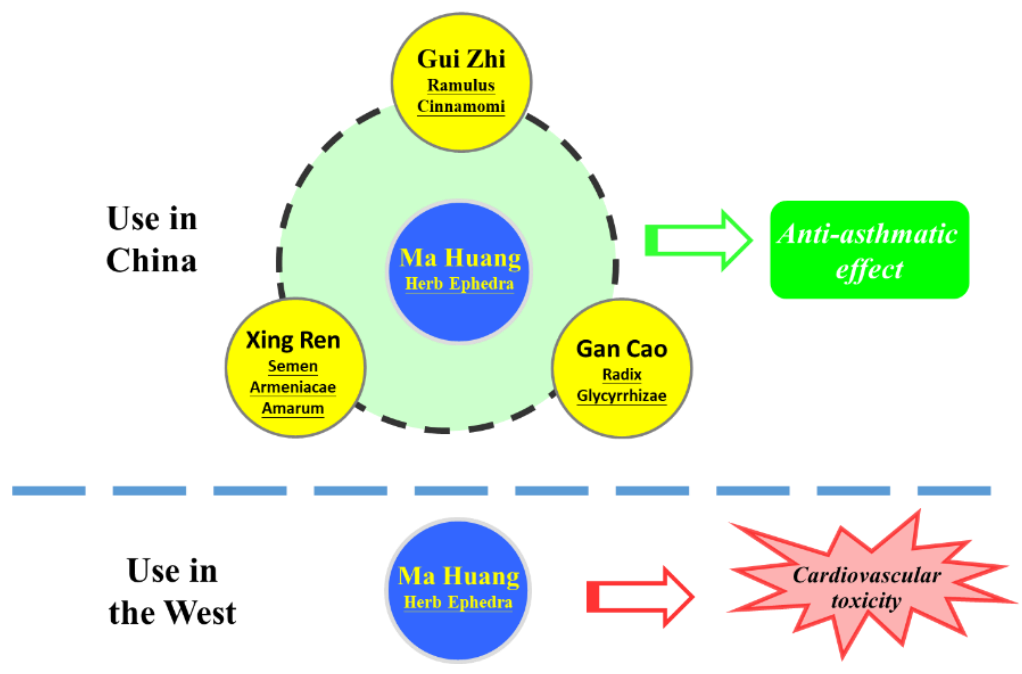

Fig. 3. In TCM, herbs are combined together in a specific proportion to treat disease efficiently with low side effects. This is exemplified with the herb formula that contain Ma Huang as the most effect herb to treat asthma. In the West, Ma Huang is used alone and in relative high dose that is prone to give cardiovascular side effect.

\section{How to connect Western and Eastern medicine}

To connect Western and Eastern medicine, we should not concentrate on differences but try to find similarities. As will be discussed in chapter one, there is a striking similarity between Western medicine and Eastern medicine when we look at them from an energy perspective. As outlined in this chapter, in both worlds, opposing forces provide the energy that flows through networks in an organism, which fuels life. In this concept, health is the ability of an organism to maintain the balance between these opposing forces, i.e. homeostasis (West) and harmony (East), which creates resilience. Moreover, strategies used to treat diseases are strikingly alike. Redox modulating compounds and TCM are added to increase or strengthen connections in the network to finally adjust the flow of energy and regain its balance.

So the energy perspective provides a basis to integrate Eastern and Western medicine. It shows us what we should study. For example, we need to extend our knowledge on how various redox modulators will have different effects in the network. Moreover, in the West we might benefit more from the dynamic interaction between molecules, one of the fundaments of TCM. Another opportunity is to study other forms of energy, e.g. light. An interesting finding is that TCM "corrects" the light transmitted by the body [26]. This cannot be explained with the Western reductionistic approach, yet. There are numerous other mysterious "forces" in Eastern medicine and other types of traditional medicine that lack a "Western scientific basis", and therefore are left unused and might even be lost. Undoubtedly, medicine will improve when East and West become more connected, and we get the best of both worlds (Fig. 4). 


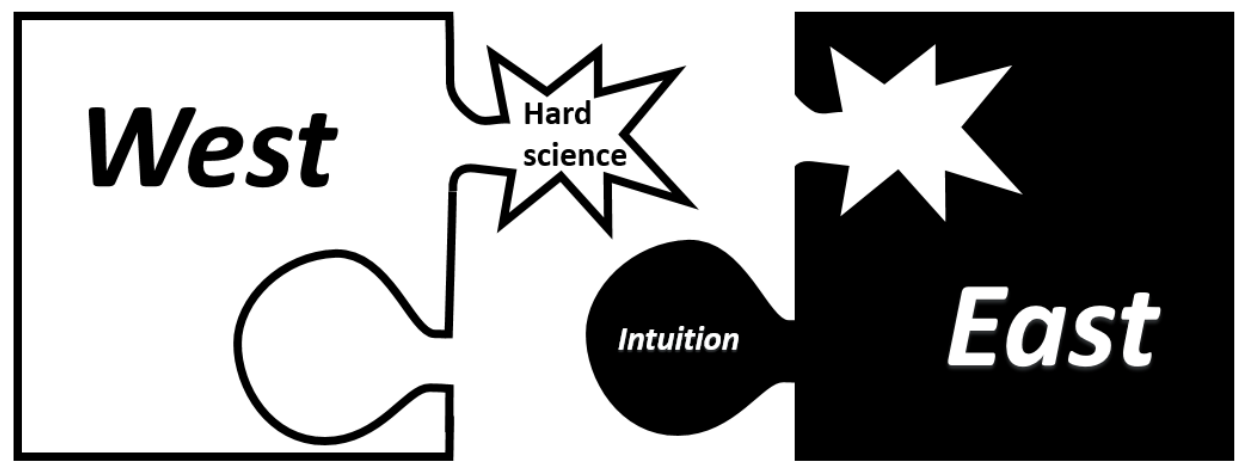

Fig. 4. Connecting West and East. Eastern medicine is more intuitive, while Western medicine is more scientific. Although worlds apart, West and East world should be combined to solve the puzzle.

\section{Aim and outline of the thesis}

In order to connect Western and Eastern medicine, we explored Eastern medicine from Western perspective. In chapter one, a bridge between Eastern and Western medicine is made by looking through the perspective of energy. In chapter two, we explore how the use of Ma Huang in TCM may protect against the side effect of Ma Huang observed in the in the West. In chapter three, we investigate the potential anticholinergic accumulation of TCM, as TCM contains many compounds that are expected to bind to muscarinic receptors. In chapter four and chapter five, the redox modulating properties of (-)-epicatechin and quercetin are further unraveled. 


\section{Preference}

1. van der Greef, J.; van Wietmarschen, H.; Schroën, J.; Wang, M.; Hankemeier, T.; Xu, G. Systems biologybased diagnostic principles as pillars of the bridge between Chinese and Western medicine. Planta medica 2010, 76, 2036-2047.

2. Schroën, Y.; van Wietmarschen, H.A.; Wang, M.; van Wijk, E.P.; Hankemeier, T.; Xu, G.; van der Greef, J. East is East and West is West, and never the twain shall meet. Science 2014, 346, S10-S12.

3. World Health Organization. Global strategy and plan of action on public health, innovation and intellectual property. Avaliable online: https://www.who.int/phi/publications/gspa-phi/en/ (Acessed on 26 Janary 2011).

4. Bast, A.; Haenen, G.R. Ten misconceptions about antioxidants. Trends in pharmacological sciences 2013, 34, 430-436.

5. Kim, E.J.; Chen, Y.; Huang, J.Q.; Li, K.M.; Razmovski-Naumovski, V.; Poon, J.; Chan, K.; Roufogalis, B.D.; McLachlan, A.J.; Mo, S.L. Evidence-based toxicity evaluation and scheduling of Chinese herbal medicines. Journal of ethnopharmacology 2013, 146, 40-61.

6. Rang, H.P.; Ritter, J.M.; Flower, R.J.; Henderson, G. Rang \& Dale's pharmacology; Elsevier Health Sciences: China, 2014.

7. Alberts, B.; Bray, D.; Hopkin, K.; Johnson, A.D.; Lewis, J.; Raff, M.; Roberts, K.; Walter, P. Essential cell biology; Garland Science: New York, US, 2013.

8. Weseler, A.R.; Ruijters, E.J.; Drittij-Reijnders, M.-J.; Reesink, K.D.; Haenen, G.R.; Bast, A. Pleiotropic benefit of monomeric and oligomeric flavanols on vascular health-a randomized controlled clinical pilot study. PLoS One 2011, 6, e28460.

9. Hooper, L.; Kay, C.; Abdelhamid, A.; Kroon, P.A.; Cohn, J.S.; Rimm, E.B.; Cassidy, A. Effects of chocolate, cocoa, and flavan-3-ols on cardiovascular health: a systematic review and meta-analysis of randomized trials. The American journal of clinical nutrition 2012, 95, 740-751.

10. Tang, W.; Eisenbrand, G. Chinese drugs of plant origin: chemistry, pharmacology, and use in traditional and modern medicine; Springer Science \& Business Media: Heidelberg, Germany, 2013.

11. Jin, R.; Lin, Z.J.; Xue, C.M.; Zhang, B. An improved association-mining research for exploring Chinese herbal property theory: based on data of the Shennong's Classic of Materia Medica. Journal of integrative medicine 2013, 11, 352-365.

12. Tao, W.; Xu, X.; Wang, X.; Li, B.; Wang, Y.; Li, Y.; Yang, L. Network pharmacology-based prediction of the active ingredients and potential targets of Chinese herbal Radix Curcumae formula for application to cardiovascular disease. Journal of ethnopharmacology 2013, 145, 1-10.

13. van Wietmarschen, H.A.; van der Greef, J.; Schroën, Y.; Wang, M. Evaluation of symptom, clinical chemistry and metabolomics profiles during Rehmannia six formula (R6) treatment: an integrated and personalized data analysis approach. Journal of ethnopharmacology 2013, 150, 851-859.

14. Jóźwiak-Bebenista, M.; Nowak, J.Z. Paracetamol: mechanism of action, applications and safety concern. Acta poloniae pharmaceutica 2014, 71, 11-23.

15. Croft, S.L.; Sundar, S.; Fairlamb, A.H. Drug resistance in leishmaniasis. Clinical microbiology reviews 2006, 19, 111-126.

16. Haldar, K.; Bhattacharjee, S.; Safeukui, I. Drug resistance in Plasmodium. Nature reviews microbiology 2018, 16, 156.

17. Vrolijk, M.F.; Opperhuizen, A.; Jansen, E.H.; Bast, A.; Haenen, G.R. Anticholinergic accumulation: A slumbering interaction between drugs and food supplements. Basic \& clinical pharmacology \& toxicology 2015, 117, 427-432.

18. Ioannidis, J.P. Why most clinical research is not useful. PLoS medicine 2016, 13, e1002049.

19. Singh, I. Bad boys, good mothers, and the "miracle" of Ritalin. Science in context 2002, 15, 577-603.

20. Bierly, P.; Chakrabarti, A. Generic knowledge strategies in the US pharmaceutical industry. Strategic management journal 1996, 17, 123-135.

21. Hong, H. Principles of Chinese Medicine: A Modern Interpretation; World Scientific: London, England, 2015.

22. van der Greef, J.; van Wietmarschen, H.; Schroën, Y.; Babouraj, N.; Trousselard, M. Systematic approaches to evaluation and integration of eastern and Western medical practices. Medical acupuncture 2015, 27, 384395.

23. U.S. Food and Drug Administration. Regulation prohibiting sale of dietary supplements containing ephedrine alkaloids and reiterates its advice that consumers stop using these products. Avaliable online: http://www.fda.gov/NewsEvents/Newsroom/PressAnnouncements/2004/ucm108242.htm (accessed on 6 February 2004). 
24. Yao, Y.; Zhang, X.; Wang, Z.; Zheng, C.; Li, P.; Huang, C.; Tao, W.; Xiao, W.; Wang, Y.; Huang, L. Deciphering the combination principles of Traditional Chinese Medicine from a systems pharmacology perspective based on Ma-huang Decoction. Journal of ethnopharmacology 2013, 150, 619-638.

25. Pipe, A. Efficacy and safety of ephedra and ephedrine for weight loss and athletic performance. Clinical Journal of sport medicine 2004, 14, 188-189.

26. Rosch, P.J. Bioelectromagnetic and subtle energy medicine. Annals of the New York academy of sciences 2009, 1172, 297-311. 


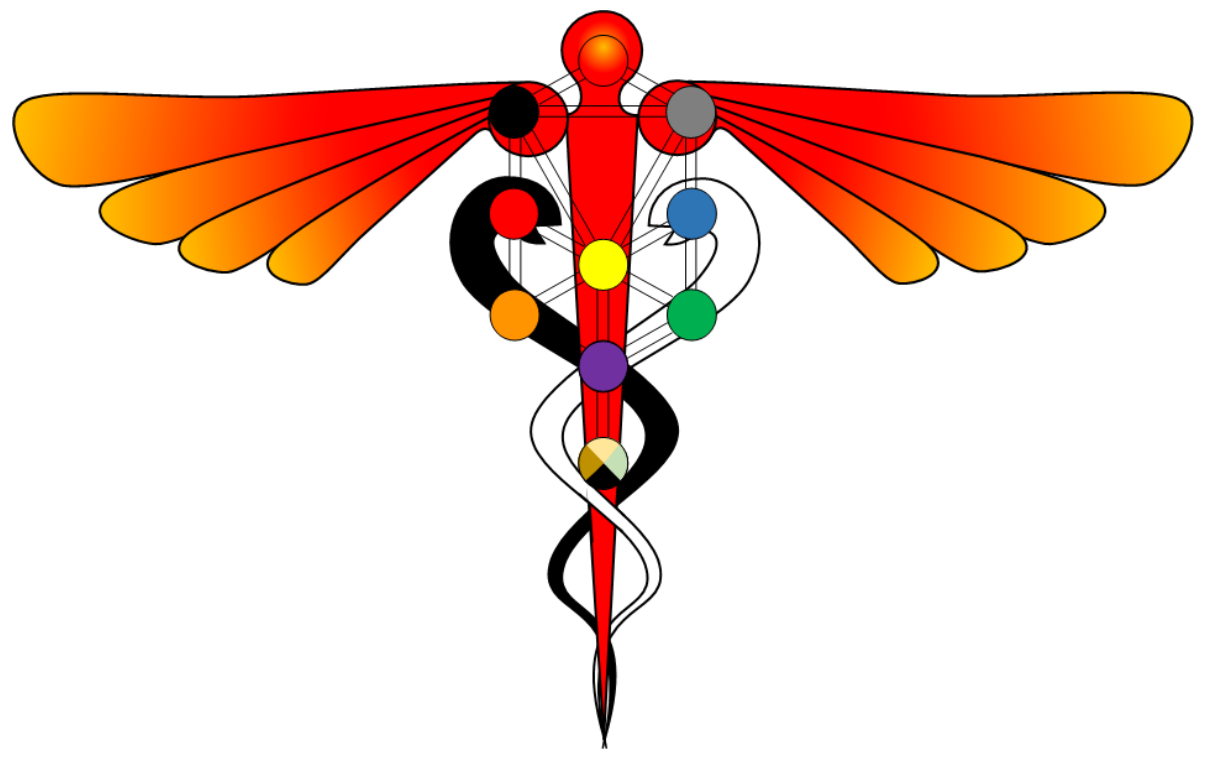




\section{Chapter 1}

\section{Connecting Western and Eastern medicine from an energy perspective}

Ming Zhang, Mohamed Moalin, Lily Vervoort, Zheng Wen Li, Wen Bo Wu and Guido R.M.M. Haenen

International Journal of Molecular Science. 2019; 20(6): 1512. 


\begin{abstract}
Although Western medicine and Eastern medicine are worlds apart, there is a striking overlap in the basic principle of these types of medicine when we look at them from the perspective of energy. In both worlds, opposing forces provide the energy that flows through networks in an organism, which fuels life. In this concept, health is the ability of an organism to maintain the balance between these opposing forces, i.e., homeostasis (West) and harmony (East), which creates resilience. Moreover, strategies used to treat diseases are strikingly alike, namely adjusting the flow of energy by changing the connections in the network. The energy perspective provides a basis to integrate Eastern and Western medicine, and opens new directions for research to get the best of both worlds.
\end{abstract}

Keywords: western medicine; eastern medicine; opposing forces; redox; Yin-Yang; resilience

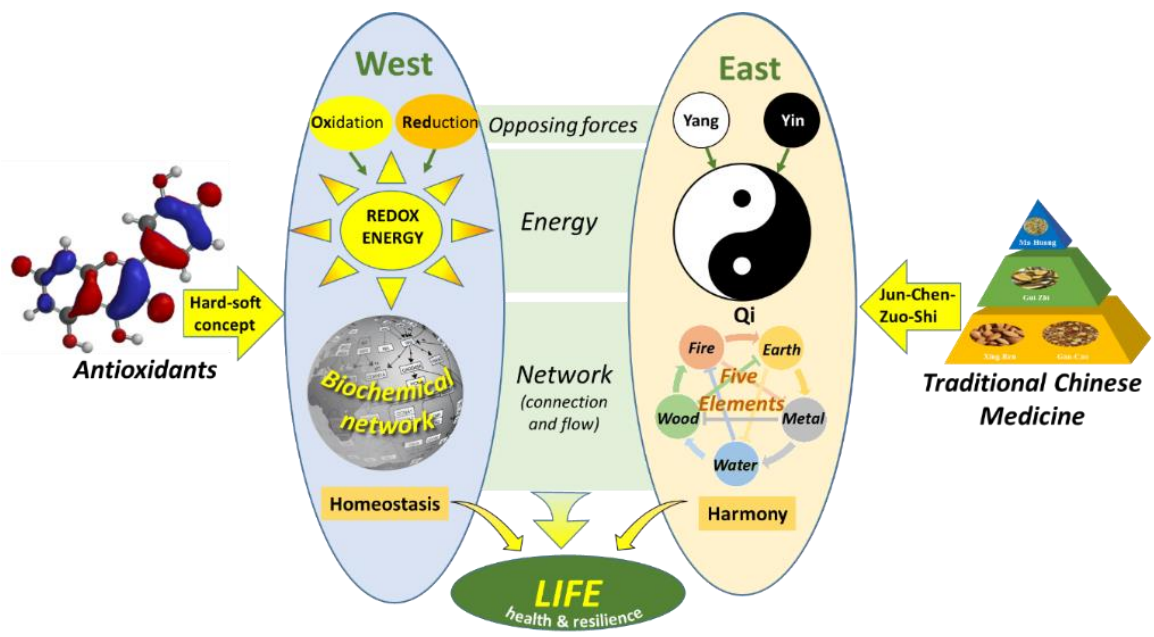




\section{Introduction}

Some people hope that, when their Earthly life is finished, they go to heaven and are spared from burning in hell. What most people do not know is that we do not have to worry about burning in hell; we already burn on Earth. This burning is caused by an unusual suspect, namely oxygen. Most people think we need oxygen and that oxygen is essential for our life. This is correct, but oxygen is also highly reactive and therefore toxic. Because of oxygen, we slowly burn like a candle. This can be exemplified by the development of age pigments. Age pigments are burned proteins that our body cannot remove and therefore accumulate and become toxic [1]. Another example is the burning of DNA that can give rise to cancer [2].

\section{Opposing Forces}

The burning caused by oxygen is called "oxidation". Luckily, there is also a counter force, namely "reduction". The first letters of reduction and oxidation combine to give "redox". Redox describes the equilibrium between the oxidizing and reducing force (Fig. 1). As also described in the Introduction, redox energy is the driving force of life; however, redox energy also causes damage. It contains both good and bad.

That everything contains good and bad was coined in the West by Paracelsus in the 15th century who stated that: "A very good thing worth to be acquired, first has to be separated from the bad. The art is such that nothing good can be acquired without a price. To get what you want, you must face also that you do not want" [3]. Within this paradox lies another paradox. An example of this double paradox is that oxidative damage to DNA may, by chance, give rise to a mutated organism that is better adapted to its environment [4]. This is the fundament of the "survival of the fittest" theory of evolution. Thus, "damage" is also essential for survival, resilience and progression.

In the East, it was realized much earlier than in the West that everything contains opposing forces. This is best symbolized by the Tai Ji symbol that visualizes the balance between Yin and Yang. In the black part of the circle, there is a small white circle $[5,6]$. This visualizes one of the double paradoxes in the symbol. Actually, the Tai Ji symbol is one of the first fractals made by man. The paradoxical nature of oxygen might therefore be better symbolized using the Tai Ji symbol as done in Fig. 2, compared to the Western chemical symbol of oxygen.

Yin and Yang are opposing forces that control life, as well as the universe. Yin mainly influences the physical, material aspect and Yang domains the psychological, spiritual aspect of an organism. However, similar to reduction and oxidation, they interact and balance each other. When Yin rises, Yang declines, and when Yin declines, Yang rises [79]. The opposing forces form the vital energy or Qi that circulates in our body. The organs work together by regulating and preserving Qi through the channels called meridians and collaterals. Diseases will happen when the Qi creates disharmony. Treatment aims to correct the Yin-Yang balance, thus Qi is readjusted and harmony is restored [10]. 


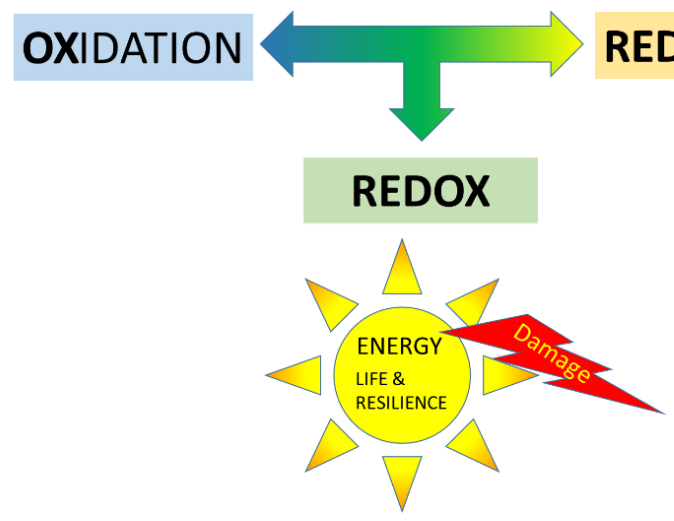

Fig. 1. Redox, the equilibrium between oxidation and reduction, is the energy source for life, as well as damage.

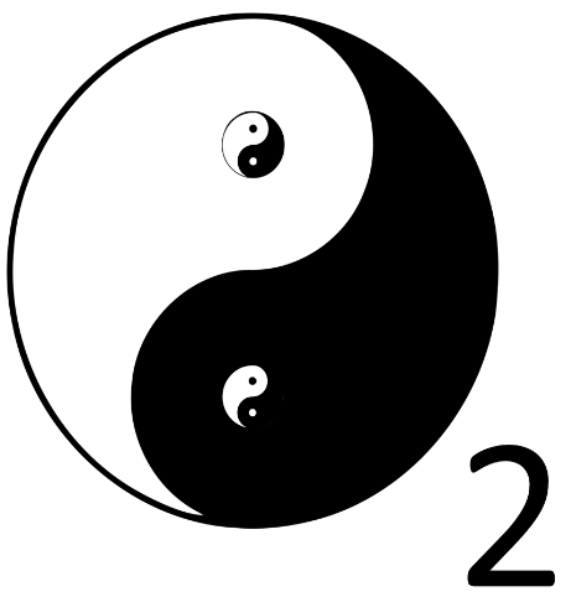

Fig. 2. The symbol that might visualize the nature of oxygen more accurately than the Western chemical symbol $\left(\mathrm{O}_{2}\right)$. Oxygen is essential for life but it is also reactive and therefore toxic. This dual nature is resembled in the Tai Ji symbol.

\section{Western Concept of Health}

In our life, the redox energy changes (Fig. 3). In a healthy body, this redox energy is high and kept within very strict limits in our body. We call this homeostasis, a harmony comparable to the Yin-Yang balance. Health can be defined as the ability to adapt to challenges [11]. When we have stress, a challenge, there is an attack on our energy. The energy goes down, but our body has resilience to absorb the challenge and bounce back. In the rebound, there is even a temporary improvement and you build up resistance [12-15]. Thus, a little bit of bad is good for you, which is in line with the double paradox described above. We do have a problem when the challenge is intense and prolonged. For example, 
due to a chronic illness, the energy decreases and reaches a new equilibrium at a lower energy level [16]. In this condition, we can no longer cope with even a small challenge. All energy might flow away and then it is the end.

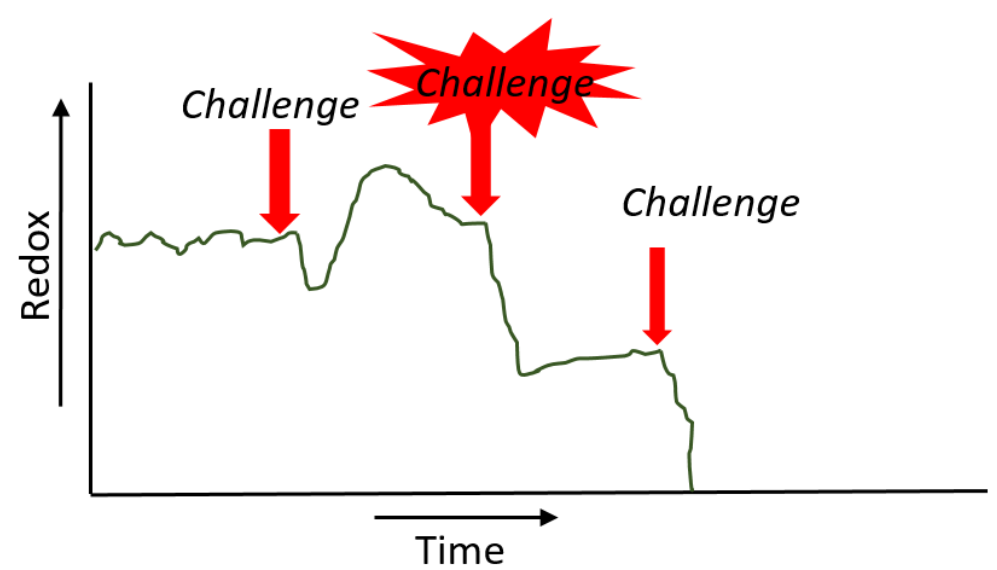

Fig. 3. Change of the redox energy in our life. The explanation is given in the text.

Where does this resilience of our body come from? In Western science, the body is made up of molecules, and all molecules are closely linked together. These connections can be visualized by the network plots that are generated in system biology (Fig. 4) [17,18]. A molecule can be converted into another molecule, in a process often catalyzed by enzymes and controlled by various other molecules. The energy for these connections comes from redox. Together, these connections form an elastic safety net that easily can absorb a challenge and even can give a rebound that increases resilience. In a sick organism, many connections are lost, and the safety net becomes fragile. This will reduce resilience [19]. The importance of connections is easily remembered with an elegant mnemonic. The first two letters of "wellness" give "we". When "we" are together, "we" are connected and healthy. This is true in every society and culture. The first letter of "illness" gives "i". When "I" am alone, "I" am isolated and this is not healthy [20]. 


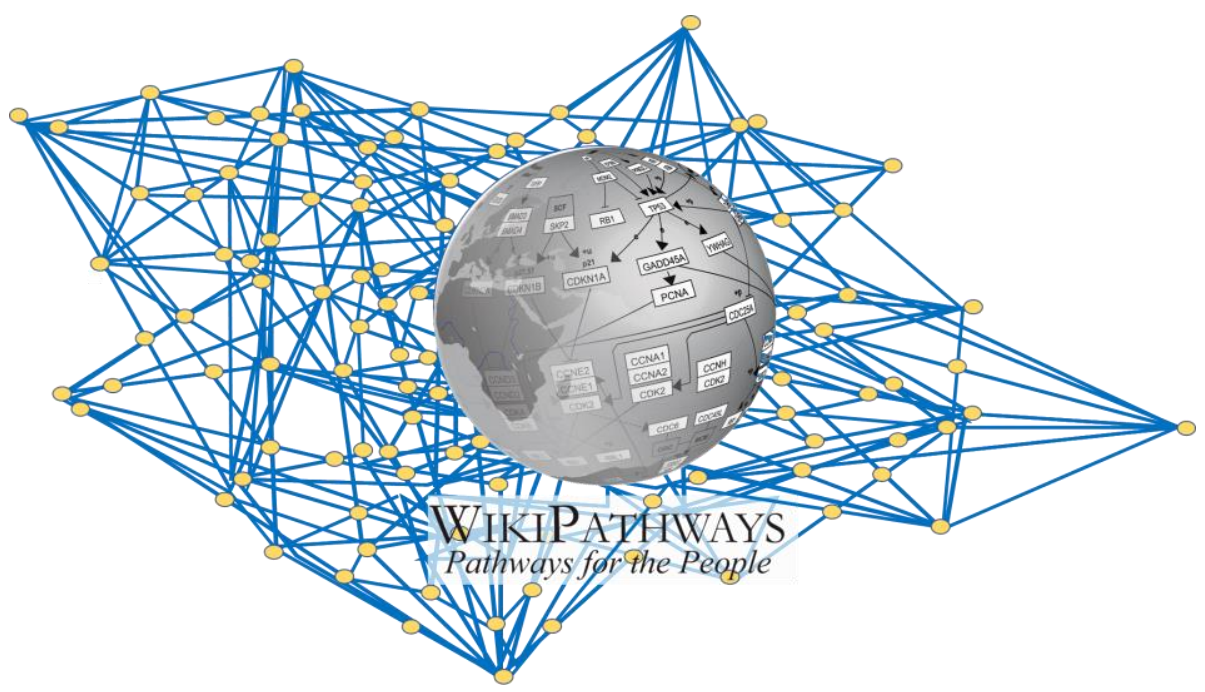

Fig. 4. The network formed by biochemical reactions. The background gives a schematic impression of how molecules (yellow dots) in a cell are connected by chemical reactions (blue lines), illustrating that together they form an "elastic safety net" that can deal with challenges. An overview of these reactions is given by the interactive "WikiPathways" platform (Available online: https://en.wikipedia.org/wiki/WikiPathways) an open community resource dedicated to collect all interactions among molecules in a cell.

\section{Eastern Concept of Health}

Connections are also very important for resilience and health in the East. In Chinese philosophy, all organisms are made up of the five symbolic elements: wood, fire, earth, metal and water (Fig. 5). These five elements interact and compensate each other to find the proper balance. An element can generate another element in the generation cycle. In the destruction cycle, an element restrains another element [21]. The interaction between the elements is governed by the Yin and Yang balance. Each organism is constantly striving for an optimal dynamic balance or harmony of these five elements, which is associated with a healthy condition. In this condition, all vital organs of the body can cope with challenges, which gives resilience. An imbalance between the elements in this network will be manifested as a disease. 


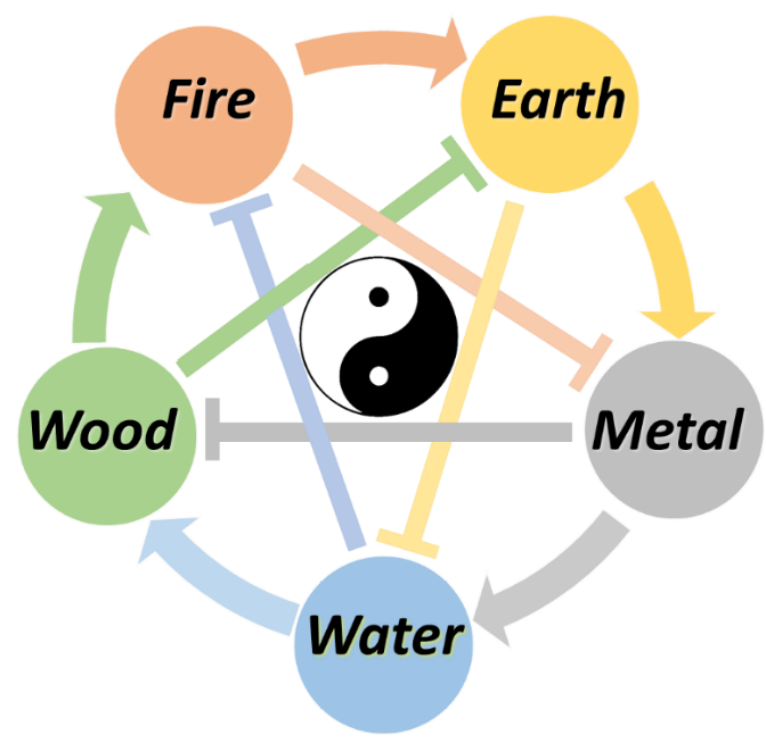

Fig. 5. Basic principle of the five elements theory. Each element is generated by another (generation cycle), but also controlled by another one (destruction cycle). Wood generates fire, fire generates earth, earth generates metal, metal generates water and water generates wood, as depicted by the colored curved arrows. Meanwhile, wood controls earth, earth controls water, water controls fire, fire controls metal and metal controls wood, as depicted by the colored $\mathrm{T}$ arrows. The interaction between the elements in this network is governed by Yin and Yang, symbolized by the Tai Ji symbol.

\section{Connecting East and West}

Despite the big differences between Eastern and Western medicine, apparently, the above shows that the basic principle used are strikingly similar. Both make use of opposing forces that create energy that flows through a network to fuel life. As outlined in the next paragraph, both types of medicine make use of this concept to treat diseases or increase health. To increase health, both aim to strengthen connections or make new ones to readjust the balance, i.e., homeostasis (West) or harmony (East), and strengthen the network (resilience).

\subsection{Western medicine}

In Western science, connections are made when molecules react with each other and this can offer protection [22-24]. An important question is why some connections are made while others are not; why reactions between some molecules happen and others do not; and how the energy flows through the network. It appears that this can be explained according to Pearson's hard-soft-acid-base concept $[25,26]$. In the hard-soft-acid-base concept, "hard" applies to species which are relatively small, have a high charge and are weakly polarizable, and "soft" applies to species which are relatively big, have low or no charge and are strongly polarizable. Consequently, "hard" acids (electrophiles) and "hard" bases (nucleophiles) have a large orbital overlap and therefore react fast with each other. In addition, due to the large orbital overlap, "soft" electrophiles react fast with "soft" 
nucleophiles. The hard-soft-acid-base concept can also be generalized for other types of electrophiles and nucleophiles that are not necessarily an acid or a base [27].

When the energy is concentrated in one place of a molecule, similar to the focal point of a lens, this molecule becomes "hard" and very reactive. For example, in the hydroxyl radical, all the energy of the unpaired electron is located on a single atom (i.e., oxygen). It is therefore the most reactive molecule in our body (Fig. 6). The hydroxyl radical reacts quickly with virtually any molecule it encounters. This will cause an uncontrolled energy flow and inflict damage to our body. Antioxidants such as quercetin and 7-mono- $O$ - $(\beta-$ hydroxyethyl)-rutoside (monoHER) are hard electron donors that react quickly with the hydroxyl radical and other reactive oxygen species (ROS) that are hard electron acceptors. By accepting electrons, ROS are converted into relatively unreactive compounds, that do not damage the cell. For example, by accepting an electron the hydroxyl radical is converted into the hydroxyl anion that, after protonation, becomes water. By donating electrons, quercetin and monoHER are converted into their corresponding soft oxidation products, quercetin quinone methide and monoHER quinone, respectively (Fig. 6). These oxidation products act as "soft" electrophiles and selectively react with "soft" nucleophiles such as thiols instead of "hard" nucleophiles such as water. As outlined in the next paragraph, we can explain the biological effect of a challenge and the modulating effect by antioxidants using this hard-soft-acid-base concept.
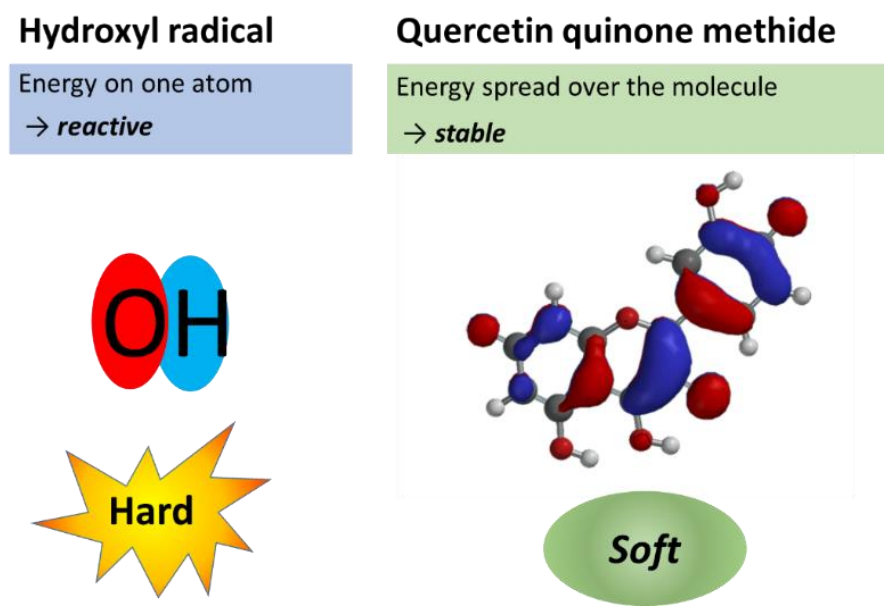

Fig. 6. Singly occupied molecular orbital of the hydroxyl radical and lowest occupied molecular orbital map of quercetin quinone methide calculated with Spartan'18. In the hydroxyl radical, all energy (in red) is concentrated on the oxygen, making it a very reactive, hard molecule. In the quercetin quinone methide molecule, the energy is spread over the molecule, thus it is a relatively unreactive, soft molecule.

A challenge on the redox energy often comes through hard energy of ROS. The hard energy of ROS will damage the cells. This is because the hard energy can react with everything in the cell including proteins, lipids and DNA, and this damage will eventually destroy the cell. There is also a sensor in the cell that is switched on by the hard energy. By switching on the sensor, the cell reacts and makes more antioxidants. This sensor is Kelchlike ECH-associated protein 1 (KEAP1). ROS can oxidize the thiol groups on KEAP1, and 
the oxidized KEAP1 will dissociate from its complex with Nuclear factor erythroid 2related factor 2 (Nrf2). The freed Nrf2 will translocate to the nucleus and stimulate the transcription of numerous detoxifying and antioxidant genes (Fig. 7A). A small challenge will eventually strengthen the defense system and increase the resilience of the cell $[28,29]$.

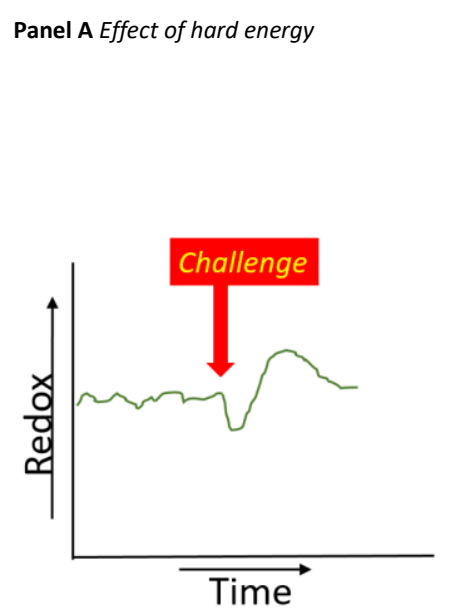

Panel B Protection by an antioxidant against hard energy

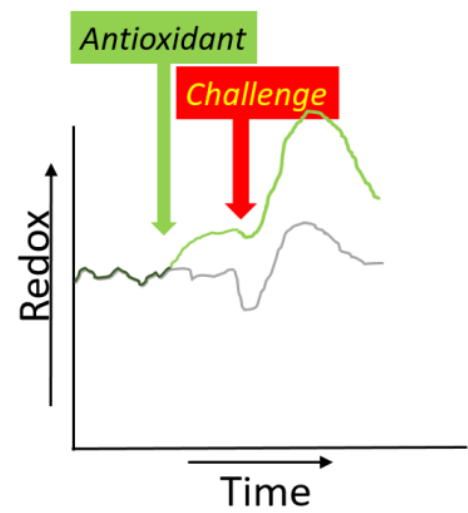

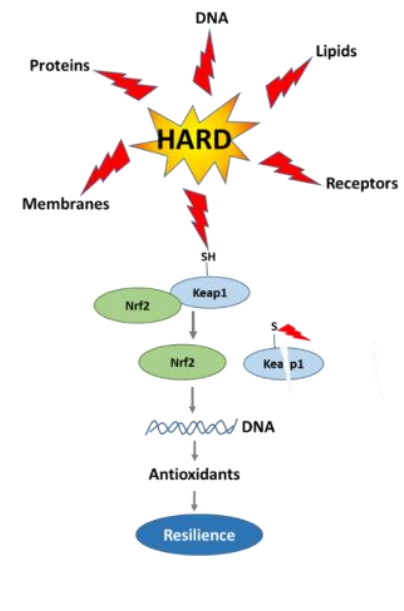

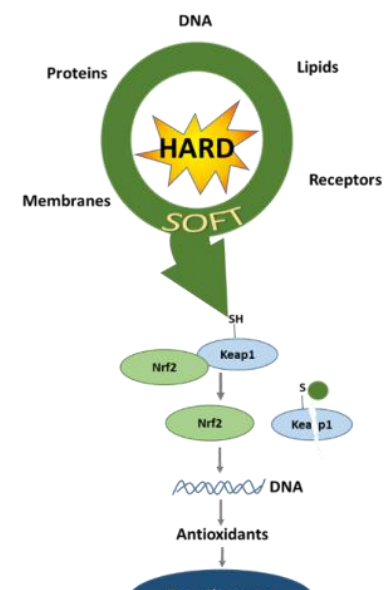

Resilience 
Fig. 7. The biological explanation for the difference in the effect of hard (A) and soft (B) energy on the redox energy. In the left figure of panel A, the dark green lines shows the change of redox energy due to a challenge. In the left figure of panel B, the green line shows how an antioxidant increases the redox energy. In the presence of an antioxidant, the damage by the challenge is less, and the rebound is higher than without antioxidant (grey line). The right figures in both panel A and panel B give the biological explanation. A "challenge" often comes from hard energy. The hard energy damages every molecule in the cell and is non-selective and inefficient in reacting with the thiol $(\mathrm{SH})$ group on Kelch-like ECH-associated protein 1 (KEAP1), that switches on the Nuclear factor erythroid 2-related factor 2 (Nrf2) pathway. An antioxidant can convert the hard energy into soft energy that gives little damage, but the soft energy is relatively efficient to react with KEAP1, switching on the Nrf2 pathway. This is because the soft energy reacts efficiently and selectively with the soft thiol (SH) group on KEAP1.

When we add an antioxidant such as monoHER, the hard energy of ROS is absorbed and softened by the formation of the soft monoHER quinone (Fig. 7B). The soft energy of this quinone does not damage DNA and other vital cell molecules, but it can turn on the sensor very well. This is because the switch on the sensor is a soft nucleophile, namely a thiol or sulfhydryl (SH) group. In line with the hard-soft-acid-base concept, a soft quinone will react efficiently and selectively with the soft thiol group on KEAP1 [12,29]. The conversion of hard into soft energy explains the direct protection as well as the efficient increase in resilience given by antioxidants. Thus, the antioxidant does not only give protection against a challenge; it also increases the rebound due to a challenge. This gives more resilience.

\subsection{Eastern Medicine}

In Eastern philosophy, when there is an imbalance between Yin and Yang, the flow of energy is incorrect and harmony is lost, which is manifested as a disease. The imbalance of energy can be corrected by acupuncture or moxibustion of meridian sites, or by Traditional Chinese Medicine (TCM). These treatments aim to readjust the Yin-Yang energy to regain the harmony of five elements.

TCM mostly consists of a combination of medicinal herbs. Their combination is based on the rule of "Jun-Chen-Zuo-Shi", known as the Four Responsible Roles (Fig. 8). Each herbal ingredient has a specific function, and their interplay makes the therapy more powerful and specific. The "Jun" (emperor) herb is the principle active herb, having the main effect in treating the disease, similar to a hard molecule. The "Chen" (minister) herb strengthens the curative effect of the Jun herb. The "Zuo" (adjuvant) herb modulates the effects of Jun and Chen and the "Shi" (messenger) herb harmonizes the effect of the other ingredients, similar to how the soft antioxidant channels the hard energy. Thus, by combining the herbs, the main therapeutic effect is enhanced, and the side effects are reduced $[30,31]$. 


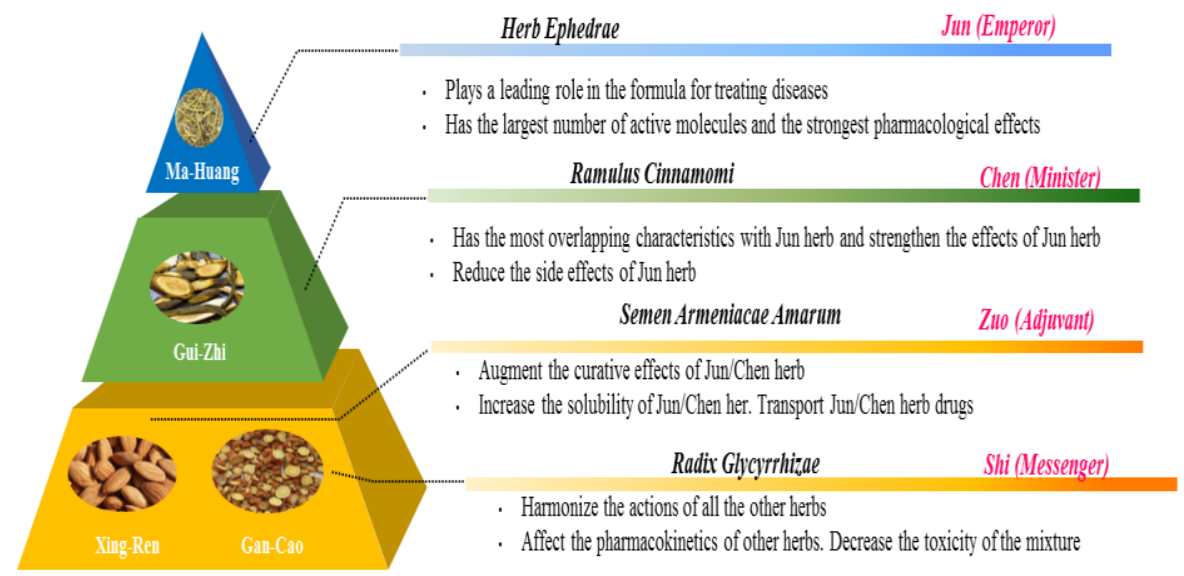

Fig. 8. diagram of the combination principle of Traditional Chinese Medicine formula, adapted from Yao et al. (2013) [30] .

For instance, in the formula Ma Huang Decoction developed to treat, e.g., asthma (Fig. 9), Ma Huang is the most effective herb and is the Jun herb. It is used to promote the sweating, and dilatate the lung. Gui Zhi serves as the Chen herb to assist Ma Huang, but it induces sweating and reduces the side effects of the Ma Huang by warming the channels to reduce headache and pantalgia. Xing Ren and Gan Cao work together as the Zuo and Shi herbs, respectively, to improve the therapeutic effects of Ma Huang and Gui Zhi. Xing Ren, which is the Zuo herb with a bitter and warm nature, increases the lung function and strengthens the sweating effect of Ma Huang. Gan Cao, the Shi herb with sweet and warm nature, harmonizes the action of the other herbs and makes the therapeutic effect of the combination more specific. Through combing herbs, the therapeutic effect of Ma Huang is enhanced, and the side effects caused by Ma Huang are also reduced to a relative safe level. The intricate interaction between compounds in TCM harmonies the heat and the energy in the body to attain good health $[32,33]$. 


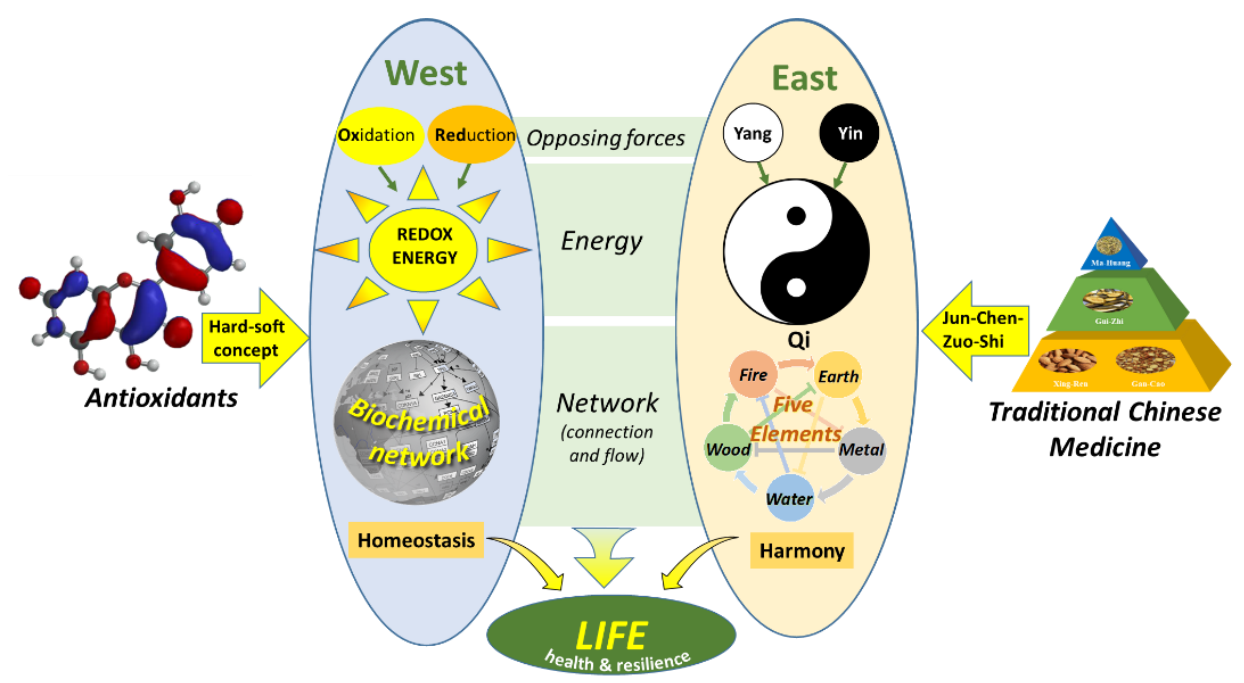

Fig. 9. The connection of Western medicine and Eastern medicine from energy perspective. In both worlds, opposing forces generate the energy that flows through networks, which fuels life. Antioxidants interact with other molecules based on the hard-soft-acid-base concept which can be used to regain homeostasis. In TCM, different herbs are combined based on the rule of "Jun-Chen-Zuo-Shi" to restore the energy of Qi in the network to regain harmony.

\section{Conclusion}

Western and Eastern philosophies of health seem to be worlds apart. In the West, an organism is reduced to a collection of molecules. This approach has been quite successful in generating effective medicines. However, the limitations of Western medicine are becoming more evident. Apparently, in the Western reductionistic approach, something is lost. This is probably best evidenced by "emotion" and "feeling" that - although essential in life - cannot be explained only by looking at molecules. Eastern medicine uses a more intuitive approach. Although, from a Western perspective, Eastern medicine is not "scientific", people have profited from this intuitive type of medicine for thousands of years and Eastern and other traditional types of medicine are still used by the majority of the people. Despite the difference, both worlds appear to be based on a similar principle when we look at Western and Eastern medicine from the perspective of energy (Fig. 9). The universal principle is that opposing forces generate the energy that flows through networks in an organism, which fuels life. This perspective might provide a basis to integrate Eastern medicine and Western medicine and opens new directions for research to fill the knowledge gaps between both worlds. 


\section{Future Perspective}

The energy perspective creates a bridge to connect Eastern and Western medicine. In the West, we might benefit more from the dynamic interaction between molecules, one of the fundaments of TCM. Using isolated arteries, we could confirm that the accompanying herbs in the Ma Huang Decotion can mitigate the side effects of Ma Huang [33]. Moreover, the dynamic interaction between several herbs in TCM on muscarinic receptor binding are also-from a Western point of view-unexpected and even contra-intuitive [34]. The energy perspective also indicates that we still need to extend our knowledge on how antioxidants that differ in hardness/softness will have different redox modulation effects [35]. Another opportunity is to study other forms of energy, e.g., light. An interesting finding is that TCM "corrects" the light transmitted by the body [36]. This cannot be explained with the Western reductionistic approach, yet. There are numerous other mysterious "forces" in Eastern medicine and other types of traditional medicine that lack a "Western scientific basis", and therefore are left unused and might even be lost. Undoubtedly, medicine will improve when East and West become more connected, and we get the best of both worlds.

\section{Acknowledgments}

With the permission of the author, Figures 1, 2, 3, 6 and 7 were adapted from "We are stardust" https://cris.maastrichtuniversity.nl/portal/files/26420841/Guido_Haenen_Oratie_definitief.p df. 


\section{References}

1. Terman, A.; Brunk, U.T. Oxidative stress, accumulation of biological 'garbage', and aging. Antioxidants \& redox signaling 2006, 8 , 197-204.

2. Valko, M.; Rhodes, C.; Moncol, J.; Izakovic, M.; Mazur, M. Free radicals, metals and antioxidants in oxidative stress-induced cancer. Chemico-biological interactions 2006, 160, 1-40.

3. Franz, I. Paracelsus-Naturkundiger unter und über Tage. Mit Brückensch. Franz Baader, 2007, 1, 33-43.

4. Sthijns, M.M.; Randall, M.J.; Bast, A.; Haenen, G.R. Adaptation to acrolein through upregulating the protection by glutathione in human bronchial epithelial cells: The materialization of the hormesis concept. Biochemical and biophysical research communications 2014, 446, 1029-1034.

5. van der Greef, J.; van Wietmarschen, H.; Schroën, J.; Wang, M.; Hankemeier, T.; Xu, G. Systems biologybased diagnostic principles as pillars of the bridge between Chinese and Western medicine. Planta medica 2010, 76, 2036-2047.

6. Tsuei, J.J. Eastern and western approaches to medicine. Western journal of medicine 1978, $128,551$.

7. Schroën, J.H. Non-linear dynamics and Chinese medicine: an essay on research models, TCM, and recent changes in modern scientific philosophy. Clinical acupuncture and oriental medicine 2002, 3, 92-98.

8. Gibert-Tisseuil, F. Reflections on traditional Chinese medicine and its pharmacopoeia. Annales pharmaceutiques francaises 1988, 56, 282-285.

9. Kolasani, A.; Xu, H.; Millikan, M. Determination and comparison of mineral elements in traditional Chinese herbal formulae at different decoction times used to improve kidney function-chemometric approach. African journal of Traditional, complementary and alternative medicines 2011, 8 .

10. Patwardhan, B.; Warude, D.; Pushpangadan, P.; Bhatt, N. Ayurveda and traditional Chinese medicine: a comparative overview. Evidence-based complementary and alternative medicine 2005, 2, 465-473.

11. Giacaman, R.; Khatib, R.; Shabaneh, L.; Ramlawi, A.; Sabri, B.; Sabatinelli, G.; Khawaja, M.; Laurance, T.; Books, Z. What is health? The ability to adapt. The lancet 2009, 373, 781-781.

12. Lemmens, K.J.; Sthijns, M.M.; van der Vijgh, W.J.; Bast, A.; Haenen, G.R. The antioxidant flavonoid monoHER provides efficient protection and induces the innate Nrf2 mediated adaptation in endothelial cells subjected to oxidative stress. PharmaNutrition 2014, 2, 69-74.

13. Cook, R.; Calabrese, E.J. The importance of hormesis to public health. Environmental health perspectives 2006, 114, 1631-1635.

14. Weis, S.; Rubio, I.; Ludwig, K.; Weigel, C.; Jentho, E. Hormesis and defense of infectious disease. International journal of molecular sciences 2017, 18, 1273.

15. Sthijns, M.M.; Weseler, A.R.; Bast, A.; Haenen, G.R. Time in redox adaptation processes: from evolution to hormesis. International journal of molecular sciences 2016, 17, 1649.

16. Bravi, M.C.; Armiento, A.; Laurenti, O.; Cassone-Faldetta, M.; De Luca, O.; Moretti, A.; De Mattia, G. Insulin decreases intracellular oxidative stress in patients with type 2 diabetes mellitus. Metabolism 2006, 55, 691-695.

17. Pico, A.R.; Kelder, T.; Van Iersel, M.P.; Hanspers, K.; Conklin, B.R.; Evelo, C. WikiPathways: pathway editing for the people. PLoS biology 2008, 6, e184.

18. Michal, G. Biochemical pathways; Spektrum, Akad. Verlag: Heidelberg, Germany, 1999.

19. He, M.; van Wijk, E.; van Wietmarschen, H.; Wang, M.; Sun, M.; Koval, S.; van Wijk, R.; Hankemeier, T.; van der Greef, J. Spontaneous ultra-weak photon emission in correlation to inflammatory metabolism and oxidative stress in a mouse model of collagen-induced arthritis. Journal of Photochemistry and photobiology B: Biology 2017, 168, 98-106.

20. The art of self healing. Availabe online: http://magazine-on-the-spot.nl/theartofselfhealing/jan-van-degreef.html (accessed on 12 January 2019).

21. Chan, K. Progress in traditional Chinese medicine. Trends in pharmacological sciences 1995, 16, 182-187.

22. Packer, J.E.; Slater, T.; Willson, R. Direct observation of a free radical interaction between vitamin E and vitamin C. Nature 1979, 278, 737.

23. Boots, A.W.; Haenen, G.R.; Bast, A. Health effects of quercetin: from antioxidant to nutraceutical. European journal of pharmacology 2008, 585, 325-337.

24. Rietjens, S.J.; Bast, A.; Haenen, G.R. New insights into controversies on the antioxidant potential of the olive oil antioxidant hydroxytyrosol. Journal of agricultural and food chemistry 2007, 55, 7609-7614.

25. Pearson, R.G. Hard and soft acids and bases. Journal of the American chemical society 1963, 85, 3533-3539.

26. Moalin, M.; van Strijdonck, G.P.; Bast, A.; Haenen, G.R. Competition between ascorbate and glutathione for the oxidized form of methylated quercetin metabolites and analogues: tamarixetin, 4' O-methylquercetin, has the lowest thiol reactivity. Journal of agricultural and food chemistry 2012, 60, 9292-9297.

27. Chattaraj, P.K.; Schleyer, P.v.R. An ab initio study resulting in a greater understanding of the HSAB principle. Journal of the American chemical society 1994, 116, 1067-1071. 
28. Lyakhovich, V.; Vavilin, V.; Zenkov, N.; Menshchikova, E. Active defense under oxidative stress. The antioxidant responsive element. Biochemistry (Moscow) 2006, 71, 962-974.

29. Lo, S.C.; Li, X.; Henzl, M.T.; Beamer, L.J.; Hannink, M. Structure of the Keap1: Nrf2 interface provides mechanistic insight into Nrf2 signaling. The EMBO journal 2006, 25, 3605-3617.

30. Yao, Y.; Zhang, X.; Wang, Z.; Zheng, C.; Li, P.; Huang, C.; Tao, W.; Xiao, W.; Wang, Y.; Huang, L. Deciphering the combination principles of Traditional Chinese Medicine from a systems pharmacology perspective based on Ma-huang Decoction. Journal of ethnopharmacology 2013, 150, 619-638.

31. Tao, W.; Xu, X.; Wang, X.; Li, B.; Wang, Y.; Li, Y.; Yang, L. Network pharmacology-based prediction of the active ingredients and potential targets of Chinese herbal Radix Curcumae formula for application to cardiovascular disease. Journal of ethnopharmacology 2013, 145, 1-10.

32. He, Y.; Gai, Y.; Wu, X.; Wan, H. Quantitatively analyze composition principle of Ma Huang Tang by structural equation modeling. Journal of ethnopharmacology 2012, 143, 851-858.

33. Zhang, M.; Schiffers, P.; Janssen, G.; Vrolijk, M.; Vangrieken, P.; Haenen, G.R. The cardiovascular side effects of Ma Huang due to its use in isolation in the Western world. European journal of integrative medicine 2018, 18, 18-22.

34. Zhang, M.; Vrolijk, M.; Haenen, G.R. The Screening of Anticholinergic Accumulation by Traditional Chinese Medicine. International journal of molecular sciences 2017, 19, 18.

35. Zhang, M.; Vervoort, L.; Moalin, M.; Mommers, A.; Douny, C.; den Hartog, G.J.; Haenen, G.R. The chemical reactivity of (-)-epicatechin quinone mainly resides in its B-ring. Free radical biology and medicine 2018, 124, 31-39.

36. Rosch, P.J. Bioelectromagnetic and subtle energy medicine. Annals of the New York academy of sciences 2009, 1172, 297-311. 


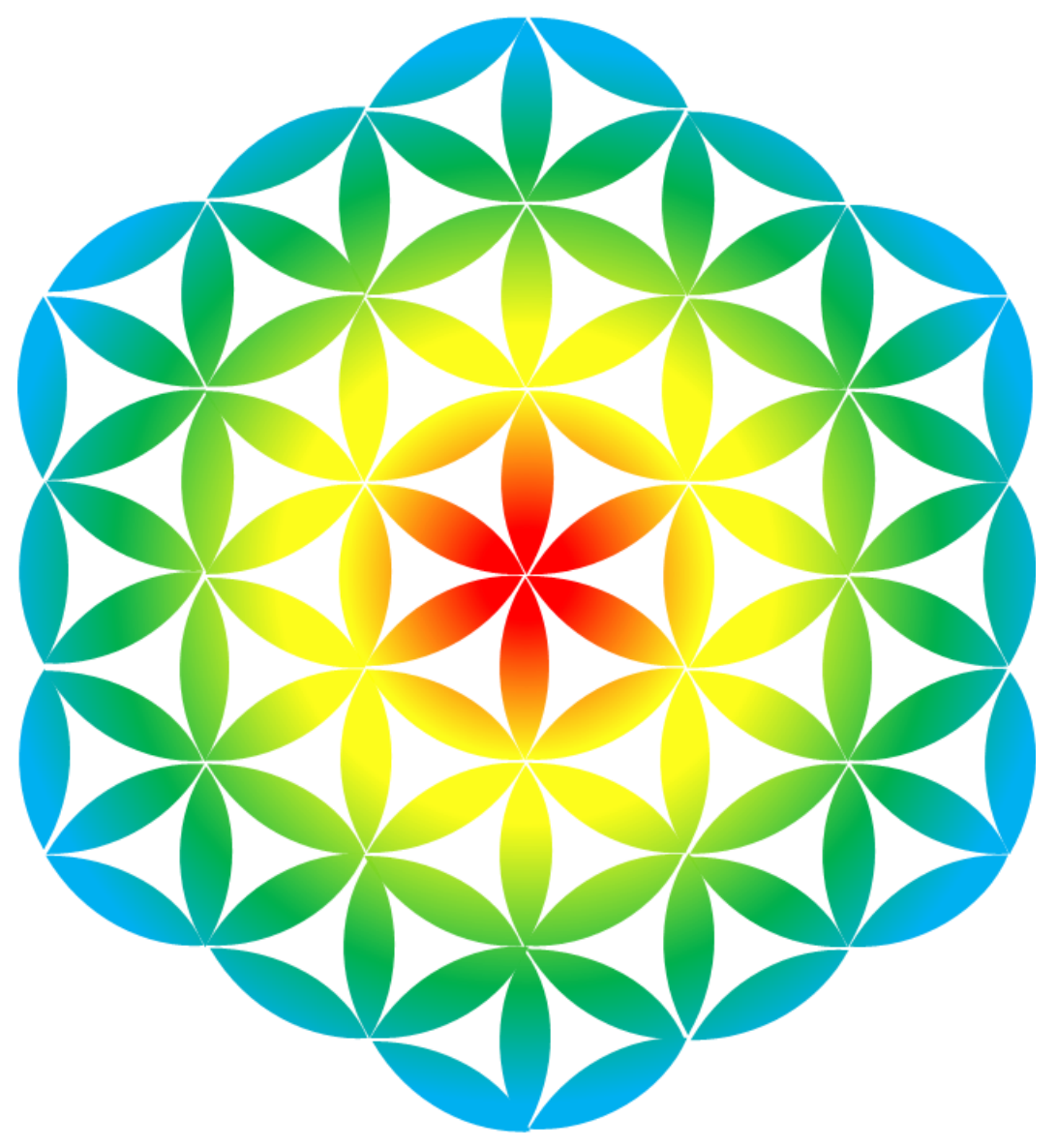




\section{Chapter 2}

\section{The cardiovascular side effects of Ma Huang due to its use in isolation in the Western world}

Ming Zhang, Paul Schiffers, Ger Janssen, Misha Vrolijk, Philippe Vangrieken and Guido R.M.M. Haenen

European Journal of Integrative Medicine. 2018; 18:18-22. 


\begin{abstract}
Introduction: An impediment for the acceptance of Traditional Chinese Medicine (TCM) in the West is that the improper use of some TCM is associated with serious side effects. This is exemplified by Ma Huang Decoction (MHD), a Ma Huang containing TCM formula used to treat asthma. The reported cardiovascular side effects often involve the improper use of Ma Huang in isolation, while Ma Huang is combined with other herbs in MHD to mitigate side effects. The aim of the study is to investigate the potential of Ma Huang as it is used in TCM to affect the cardiovascular system and compare this to Ma Huang as it is used in the West.

Methods: The contractile response of isolated arteries from human placenta to Ma Huang, MHD, and MHD without Ma Huang was used as a bioassay to evaluate their cardiovascular side effects.

Results: The contractile response by Ma Huang in isolation is much higher than that of Ma Huang in combination with the other herbs in MHD. This confirms that the interplay of the various compounds in MHD mitigates the side effects of Ma Huang. Previous studies showed that the other herbs in MHD also increase the therapeutic efficacy of Ma Huang. This indicates that the improper use of Ma Huang in isolation, also without the supervision of knowledgeable professionals, is involved in the cardiovascular side effects reported in the West.

Conclusion: To fully value its benefit, TCM should be used properly as developed over centuries in China.
\end{abstract}

Key words: Traditional Chinese Medicine, Ma Huang, Ephedrine, Toxicity 


\section{Introduction}

Traditional Chines Medicine (TCM) is the quintessence of the Chinese cultural heritage and has contributed to the Chinese Nation wealth and prosperity over the past thousands of years. TCM has become an important fundament of the Chinese health care system [1]. It is increasingly realized that TCM is also valuable for the treatment of diseases in Western societies, especially when Western drugs that have been developed fail to be effective [2]. However, to fully appreciate TCM in the West, fundamental differences between the eastern and Western culture need to be bridged.

A Western drug is designed to act selectively on a single target. The paradigm in Western medicine is that it is best to isolate active ingredients from e.g. herbs, and use the compound that is most active on one specific target [3]. In contrast, the guiding principle in TCM is that herb mixtures should be used. In the mixture, the herbs affect and balance each other to form a strong medical union to treat disease $[4,5]$.

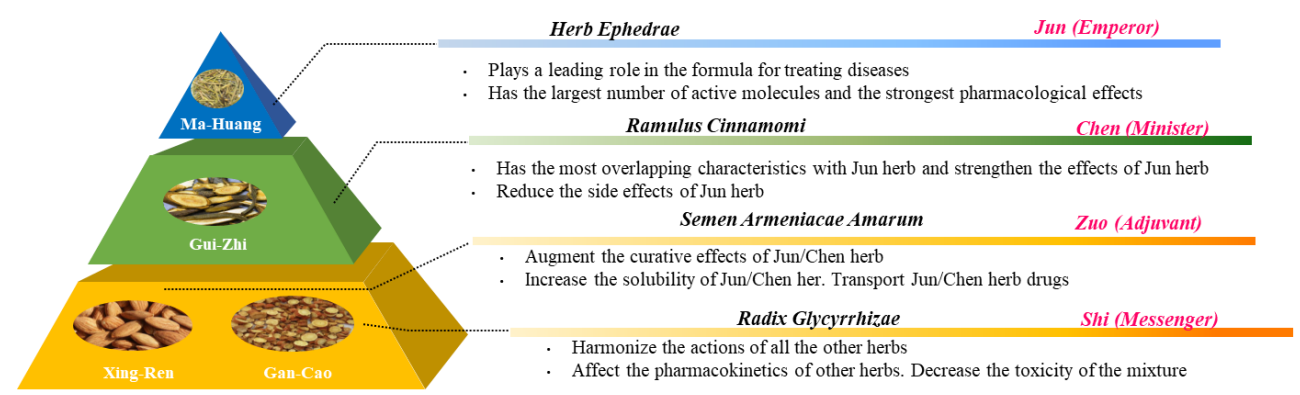

Fig.1.The diagram representing the combination principle of TCM formula, adapted from Yao et al. [6].

The combination principle of TCM is based on the rule of "Jun-Chen-Zuo-Shi" (Fig.1) [6]. Each herbal ingredient has a specific function, and their interplay makes the therapy more powerful and specific. The "Jun" herb is the principle active herb, having the main effect in treating the disease. The "Chen" herb strengthens the curative effect of the Jun herb. The "Zuo" herb modulates the effects of Jun and Chen and the "Shi" herb harmonizes the effect of the other ingredients. This work of unison can be exemplified with the wellknown herb formula, Ma Huang Decoction (MHD) used to treat asthma. MHD consists of the following individual herbs in a fixed weight ratio, Ma Huang (Herba Ephedra, Ephedra Sinica Stapf): Gui Zhi (Ramulus Cinnamomi, Cinnamomum cassia Presl): Xing Ren (Semen Armeniacae Amarum, Prunus armeniaca L.): Gan Cao (Radix Glycyrrhizae, Glycyrrhiza uralensis Fisch.) = 3:2:2:1 [7,8]. Western studies on TCM focus on the therapeutic effect. However, the main impediment for the introduction of TCM in the Western world is not the lack of evidence on the efficacy, but reports on its toxicity caused by its incorrect use [9-11]. For example, in the United States, Ma Huang has already given rise to serious cardiovascular side effects, like stroke or heart failure [12]. Therefore the 
United States Food and Drug Administration (FDA) has issued regulation on prohibiting the sale of all supplements containing Ma Huang, and reiterated its advice to stop using these products also in TCM [13], which includes MHD. However, the advice is mainly based on the misuse of Ma Huang, administered in isolation and in a relatively high dose as slimming agent without the supervision of qualified healthcare professionals $[14,15]$. This indicates that the advice involves the incorrect use of TCM, and might therefore not be justified. Nonetheless, the advice of the FDA has given TCM a bad reputation. This hampers the introduction of TCM in the West, which is a missed opportunity to profit from the benefits TCM might offer when it is used in the proper way.

The aim of the study is to examine whether Ma Huang as it is used in TCM is less prone to give cardiovascular side effects compared with the way as it is used in Western Societies. Ephedrine is the most abundant alkaloid in Ma Huang. With a comparable chemical structure to amphetamines, ephedrine works directly or indirectly by stimulating alpha adrenergic receptors, beta adrenergic receptors and trace amine associated receptors $[16,17]$. This receptor activation by ephedrine has been reported extensively and is probably involved in severe cardiovascular side effects of Ma Huang [18]. We studied the side effects in a bioassay by examining the constriction of human placenta arteries. According to the "Jun-Chen-Zuo-Shi" principle, the contractile effect of Ma Huang in MHD should be weakened by the other herbal ingredients in this formula. This would indicate that Ma Huang when used in the traditional manner confines the cardiovascular side effects, and that the total ban by the FDA is an overreaction.

\section{Materials and Methods}

\subsection{Chemicals}

All chemicals were purchased from Sigma-Aldrich (St, Louis, MO, USA). Krebs Ringer bicarbonate buffer (KRB) contains (in $\mathrm{mM}$ ): $\mathrm{NaCl} 118.2, \mathrm{KCl} 4.7, \mathrm{MgSO}_{4} \cdot 7 \mathrm{H}_{2} \mathrm{O} 1.1$, $\mathrm{KH}_{2} \mathrm{PO}_{4}$ 1.2, $\mathrm{NaHCO}_{3} 25.0, \mathrm{CaCl}_{2}$ 2.5, and glucose 5. HEPES buffered salt solution contains ( $\mathrm{pH} 7.4$, in $\mathrm{mM}$ ): $\mathrm{NaCl} 142.9, \mathrm{KCl} 4.7, \mathrm{MgSO}_{4} \cdot 7 \mathrm{H}_{2} \mathrm{O} 1.1, \mathrm{KH}_{2} \mathrm{PO}_{4} 1.2, \mathrm{CaCl}_{2} 2.5$, glucose 5 and HEPES 15. Water was prepared using a Milli-Q plotwater purification system (Millipore B.V., Amsterdam, the Netherlands).

\subsection{Preparation of herb extracts}

The raw and dry Chinese herbs were supplied by Chinaturel Import \& Export B.V. (Den Haag, the Netherlands). Ma Huang: Gui Zhi: Xing Ren: Gan Cao =3:2:2:1. Seven $\mathrm{mL}$ of $100 \%$ distilled water was added to $1 \mathrm{~g}$ of MHD. After 30 minutes at room temperature, the mixture was boiled for 1 hour. The extract was centrifuged at $5000 \times \mathrm{g}$ for $15 \mathrm{~min}$. The supernatant was filtered and stored at $-20{ }^{\circ} \mathrm{C}$. Also, an extract of only Ma Huang (the quantity of Ma Huang used was equal to the quantity of Ma Huang in MHD) was prepared according to this procedure as well as an extract of MHD without Ma Huang [19].

\subsection{HPLC analysis}

The content of ephedrine in Ma Huang and MHD were determined using an Agilent 1260 HPLC system (Agilent Technologies, Inc., USA). The constituents were separated on an Econosphere C18 column $(150 \times 4.60 \mathrm{~mm}$ i.d., $5 \mu \mathrm{m})$. The mobile phase consisted of 
acetonitrile (solvent $\mathrm{A}$ ) and $0.1 \%(\mathrm{~V} / \mathrm{V})$ trifluoroacetic acid (solvent $\mathrm{B}$ ) and changed from $0 \%$ to $15 \% \mathrm{~A}$ in $10 \mathrm{~min}$, the flow rate of $1.0 \mathrm{~mL} / \mathrm{min}$. The sample injection volume was $20 \mu \mathrm{L}$. The ultraviolet detection was set at $210 \mathrm{~nm}$. Calibration standards of ephedrine (35-140 $\mu \mathrm{g} / \mathrm{mL}$ ) were prepared in HPLC grade water. Prior to analysis, the herb extracts were filtered through a $0.2 \mu \mathrm{m}$ [20]. The concentration of ephedrine in Ma Huang and MHD was calculated based on the peak-height.

\subsection{Recording of vasomotor responses}

The vasoconstrictive activity was determined in vitro on arterial rings obtained from human placentas. The placenta arteries respond very well to various vasoconstrictive agents, including ephedrine and Ma Huang. Moreover, the placenta arteries have a human origin and are obtained from material that is otherwise discarded. The placentas were obtained after full-term pregnancies ( $>37$ weeks) of nulparria at the Academic Hospital Maastricht and Maastricht University. It was not required to ask the mothers and fathers for their informed consent as the placentas were considered rest material and patients were generally aware that rest material could be used for scientific experiments if not involving active obtainment of biological specimen. Placentas were dissected within $1 \mathrm{~h}$ after nonpathological delivery. The second and third order branches of chorionic arteries of a fresh placenta were isolated by dissection from surrounding placental tissue in HEPES buffers at room temperature. The artery was then cut into rings approximately $2 \mathrm{~mm}$ in length. Isolated arteries segments were stored in HEPES buffer for no longer than $12 \mathrm{~h}$ at $4{ }^{\circ} \mathrm{C}$.

The arterial rings were suspended in wire myographs (DMT, Aarhus, Denmark) for the recording of isometric force development. The organ chamber solution (7 $\mathrm{ml} \mathrm{KRB}$ ) was aerated with $95 \% \mathrm{O}_{2}$ and $5 \% \mathrm{CO}_{2}$ and maintained at $37{ }^{\circ} \mathrm{C}$. Each ring was streched in a stepwise manner to a diametter at which the largest contractile response to $40 \mathrm{mM} \mathrm{K}{ }^{+}$was obtained [21]. The optimal lumen diameter of the segments averaged $513 \pm 7 \mu \mathrm{m}$ and contractile responses to $62.5 \mathrm{mM} \mathrm{K} \mathrm{K}^{+}$averaged $1.6 \pm 0.2 \mathrm{~N} / \mathrm{m}$. Vascular functions were assessed as follows: Arterial rings were stimulated with $62.5 \mathrm{mM} \mathrm{K} \mathrm{K}^{+}$to determine the receptor independent contraction. Then the arterial segments were washed and equilibrated for $30 \mathrm{~min}$. Concentration response curves of Ephedrine, Ma Huang, MHD, and MHD without Ma Huang and of extracts were constructed.

\subsection{Data analysis}

Results are shown as mean \pm standard error of the mean (S.E.M.); $n$ is the number of independent experiments that were performed using different placentas. Contractile responses of the herb extracts and ephedrine are expressed as percentage of the contraction achieved by $\mathrm{K}^{+}(62.5 \mathrm{mM})$ that was recorded just before determining the contractile response of the extracts or ephedrine. Concentration-response curves were fitted to nonlinear regression curve (GraphPad Prism 5). One-way analysis of variance (ANOVA) was used for the comparison of concentration-response curves by IBM SPSS Statistics 24. Tukey's post hoc t-tests were used to compare multiple groups. A P-value $<0.05$ was considered as a significant difference. 


\section{Results}
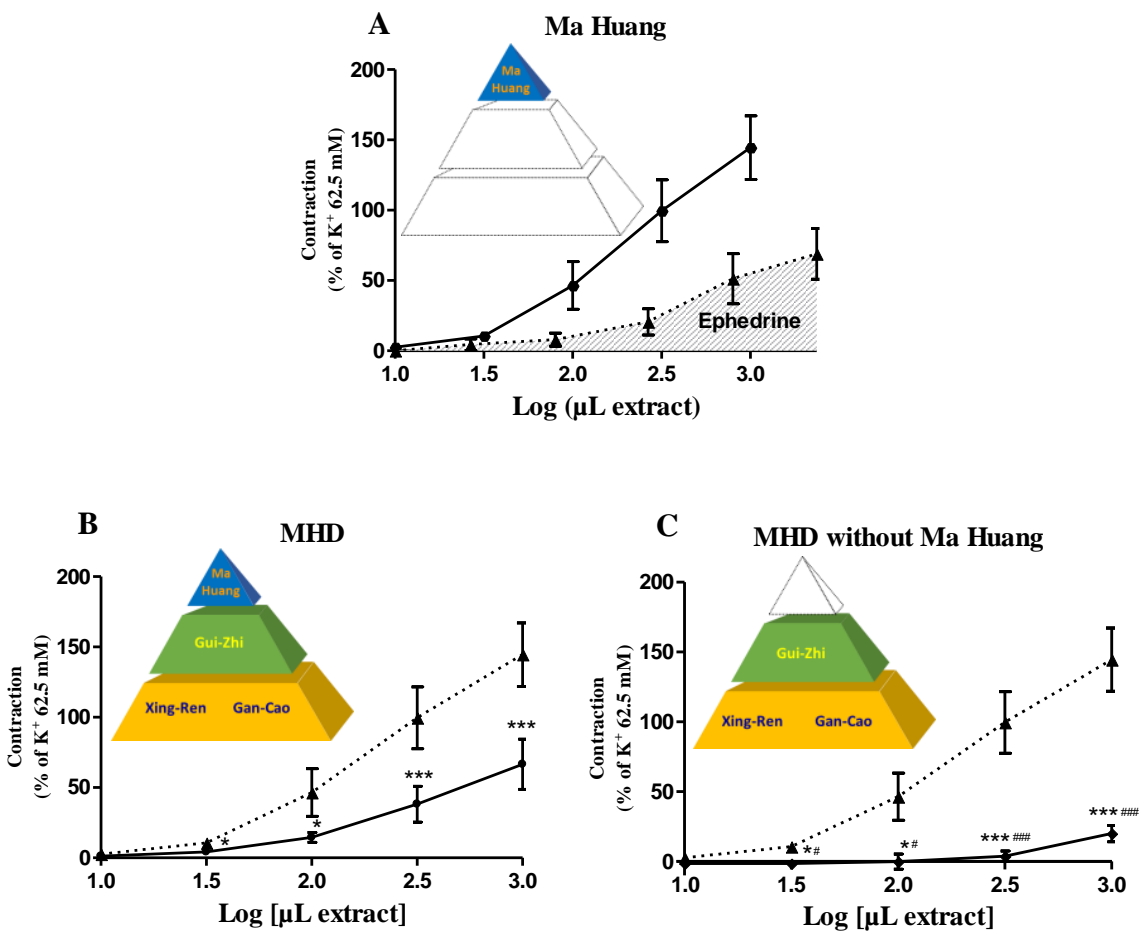

Fig.2. Contraction of arteries to the extract of Ma Huang (A), MHD (B), MHD without Ma Huang (C) (n=7). The contractions are given as the mean \pm S.E.M. In panel A, the contraction evoked by ephedrine in the concentration present in the Ma Huang extract, is depicted by dotted line. Contractions are expressed as percentage of the contraction by $\mathrm{K}^{+}(62.5 \mathrm{mM})$. The contracrion of MHD (panel B) and MHD without Ma Huang (panel C) are compared to that of Ma Huang which was depicted with a dotted line. $\mathrm{P}$ values are: ${ }^{*} \mathrm{P}<0.05,{ }^{* * *} \mathrm{P}<0.001$. The effect of MHD without M Hang was compared to MHD (panel C). P values are: ${ }^{\#} \mathrm{P}<0.05,{ }^{\# \# \#} \mathrm{P}<0.001$. 


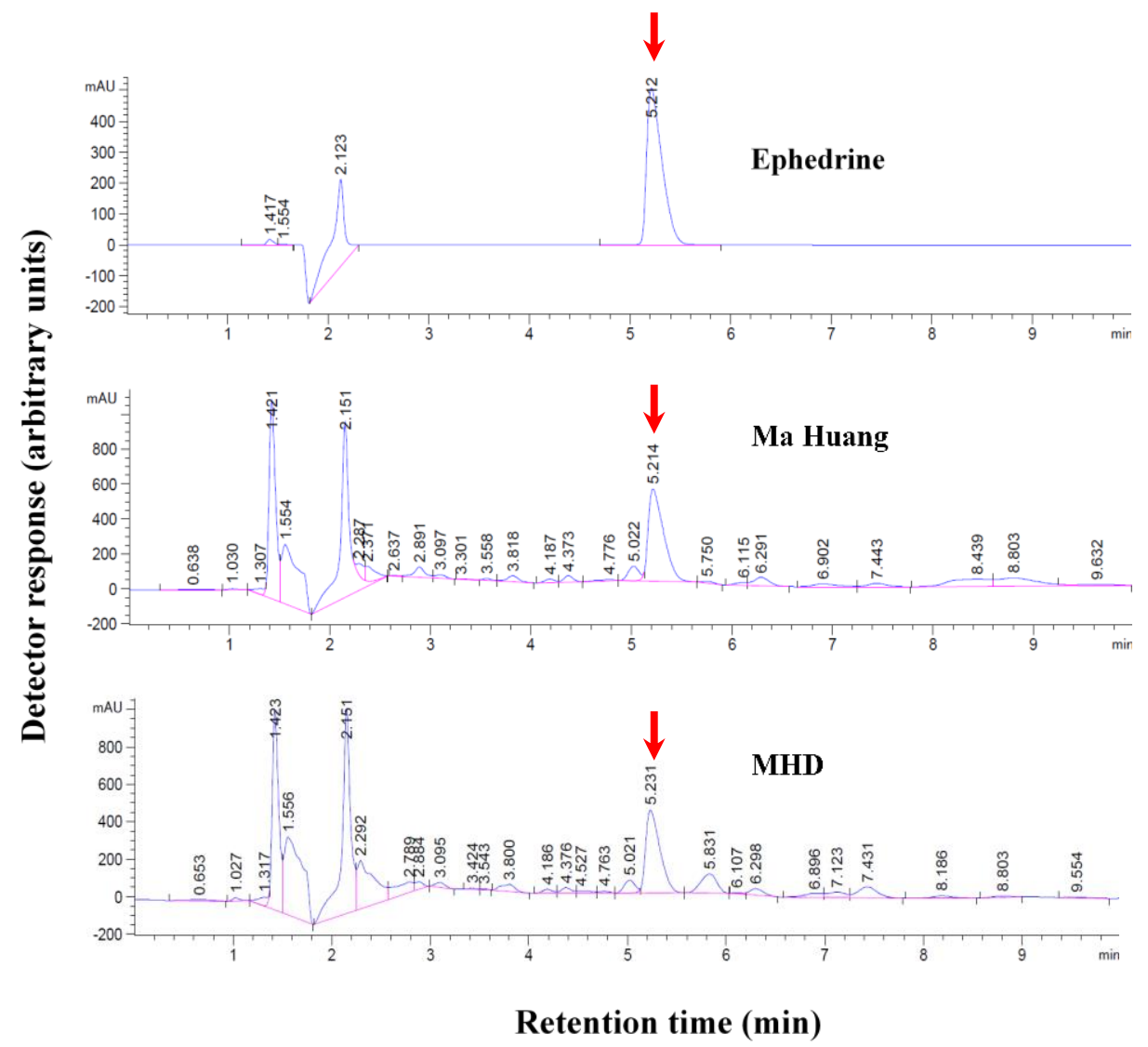

Fig.3. The HPLC chromatograms of Ephedrine $(140 \mu \mathrm{g} / \mathrm{mL})$ and extracts of Ma Huang and MHD. The peak in the Chromatogram of ephedrine is indicated by the arrow. The retention time of ephedrine is $5.2 \mathrm{~min}$.

The contractile effect of the extract of Ma Huang and MHD on placental arteries was determined. It was found that Ma Huang used in isolation induced a potent constriction of the arteries (Fig. 2A). Remarkably, the effect of Ma Huang is much less when it is used in combination with other herbs in MHD (Fig. 2B). The contractile response of MHD without Ma Huang is practically absent, which confirms the pivotal role of Ma Huang in the contraction (Fig. 2C).

The maximal contractile effect of Ma Huang in isolation is 3 to 4 times higher than that of MHD. That the contractile effect of MHD is less indicates that the herbs next to Ma Huang indeed mitigate the side effects as outlined in the rule of "Jun-Chen-Zuo-Shi". The misuse of Ma Huang used in isolation, without the herbs that can mitigate the effect of Ma Huang, can explain why Ma Huang used is prone to cause cardiovascular side effects. Our bioassay indicates that the side effects are less when Ma Huang is used according to the way it has developed over several centuries in TCM.

As also stated above, the contractile effect of Ma Huang is ascribed mainly to the presence of ephedrine. The ephedrine activates receptors on the artery causing the constriction. We found that one gram of Ma Huang herb gives $8.13 \mathrm{mg}$ of ephedrine in the 
Ma Huang extract (Fig. 3). This value is in line with the content of 5.37-6.23 mg ephedrine/g herb reported in the study of Lee et al. [19]. We determined the contractile response of ephedrine and, based on the ephedrine content, calculated the contraction that would be evoked by ephedrine present in Ma Huang extract (Fig. 2A). It appeared that the contraction by ephedrine present in Ma Huang equaled only one third of the contraction of the herb extract.

The content of ephedrine in MHD is $6.83 \mathrm{mg}$ ephedrine per g Ma Huang. Apparently MHD contains less ephedrine than the Ma Huang extract, although the amount of Ma Huang used and the extraction procedure are the same. This means that the efficiency of the extraction of ephedrine was reduced when Ma Huang was combined together with other herbs, as also reported previously [22]. However, the slight reduction in the extraction efficiency of ephedrine is far less than the reduction of the contractile effect induced by the herbs that accompany Ma Huang in MHD.

\section{Discussion}

TCM has been used in China to treat diseases for over 4000 years [23]. In general, the effects of TCM are subtle, and the treatment with TCM needs time to become effective [24]. This already indicates that TCM is relatively safe. However, in the West serious side effects of TCM have been reported. It has been suggested that this also involves the lack in the West on the proper knowledge to apply TCM [2,25]. In the present study, we take the formula MHD as an example, a well-known TCM formula used to treat asthma. We evaluate its cardiovascular side effect by determining the contraction of placental arteries.

The Chinese doctors who developed the MHD recipe, have chosen Ma Huang and Gui Zhi because their pungent warm character induces sweating that dispels toxins and opens up the lung. Ma Huang is the most effective drug to deal with the disease, making it the Jun herb. Gui Zhi, acting as Chen herb, not only induces sweating, it also reduces the side effects of the Jun herb by warming the channels to reduce headache and pantalgia. Xing Ren, which is the Zuo herb, has a bitter and warm nature that increases lung function, known as "Lung-qi" and also strengthens the asthma/reducing effect of Jun herb. Gan Cao, the Shi herb with a sweet and warm nature, harmonies the action of the other herbs and makes the therapeutic effect of the combination more specific [26-28].

Our results show that Ma Huang used in isolation is very effective in contracting the arteries. The contraction by Ma Huang in our bioassay was even greater than that of the ephedrine present in Ma Huang. This corroborates the concerns made e.g. by the FDA on the uncontrolled use of Ma Huang.

Our study also shows that when Ma Huang is combined with other herbs to make MHD, the contractile response of the arteries is greatly reduced. In addition, evidence has been put forth that the other herbs in MHD also increase the therapeutic efficacy of Ma Huang [6]. Several bioactive compounds have been identified that are supposed to mediate the beneficial effects of MHD, such as ephedrine in Ma Huang [29], cinnamaldehyde in Gui Zhi [30], amygdalin in Xing Ren [31] and glycyrrhizic acid in Gan Cao [32]. However, an attempt to decipher the combination principle of MHD, revealed that far many ingredients of the combined herbs interact in complex networks. Still, the conclusion was that the discovery of these networks only partially unveiled the combination principle, which thus remains enigmatic. The major limitation for our experiments is that they are in vitro studies with extracts that should be corroborated by clinical research. Nevertheless, these in vitro 
studies help to understand why Ma Huang containing TCM can be effective in a relatively low dose, and why it is relatively safe. Ma Huang as used in China for over several centuries should not be banned based on side effects involving its improper use and misuse in the West. On the contrary, studies on TCM used correctly under supervision of knowledgably healthcare professionals should be encouraged.

The results of our study are in line with the "Jun-Chen-Zuo-Shi" principle. In MHD, the herbs were combined to, in harmony, channel the heat, and this combination does show a mitigating effect in our screening assay on side effects. It exemplifies how Eastern traditional medicine can connect to Western medical technology. In the West the limitation of the synthetic drugs acting on a single target are becoming more evident. Western strategies are shifting towards changing phenotype, making therapies more integrative similar to the approach used in Eastern medicine. Medicine will improve when East and West become more connected, and therefore research in the West on TCM needs to be stimulated.

\section{Conclusion}

In conclusion, to fully appreciate its benefits, and to use its full potential, Ma Huang should be used as developed over centuries in TCM. Our study exemplifies how the combination principle of TCM can be applied and studied with Western technology. This integrating of Eastern and Western philosophies opens new avenues for treatments, and these avenues should not be blocked but further explored. 


\section{References}

1. World Health Organiazaiton. Traditional Chinese Medicine could make "health for one" true. Availabe online: https://www.who.int/intellectualproperty/studies/trad_med_healthforone/en/ (accessed on 2003).

2. van der Greef, J.; van Wietmarschen, H.; Schroën, Y.; Babouraj, N.; Trousselard, M. Systematic approaches to evaluation and integration of eastern and Western medical practices. Medical acupuncture 2015, 27, 384395.

3. Bast, A.; Haenen, G.R. Ten misconceptions about antioxidants. Trends in pharmacological sciences 2013, 34, 430-436.

4. Che, C.T.; Wang, Z.; Chow, M.; Lam, C. Herb-herb combination for therapeutic enhancement and advancement: theory, practice and future perspectives. Molecules 2013, 18, 5125-5141.

5. Qu, Z.; Cui, J.; Harata-Lee, Y.; Aung, T.N.; Feng, Q.; Raison, J.M.; Kortschak, R.D.; Adelson, D.L. Identification of candidate anti-cancer molecular mechanisms of compound kushen injection using functional genomics. Oncotarget 2016, 7, 66003.

6. Yao, Y.; Zhang, X.; Wang, Z.; Zheng, C.; Li, P.; Huang, C.; Tao, W.; Xiao, W.; Wang, Y.; Huang, L. Deciphering the combination principles of Traditional Chinese Medicine from a systems pharmacology perspective based on Ma-huang Decoction. Journal of ethnopharmacology 2013, 150, 619-638.

7. Tao, W.; Xu, X.; Wang, X.; Li, B.; Wang, Y.; Li, Y.; Yang, L. Network pharmacology-based prediction of the active ingredients and potential targets of Chinese herbal Radix Curcumae formula for application to cardiovascular disease. Journal of ethnopharmacology 2013, 145, 1-10.

8. Jia, W.; Gao, W.y.; Yan, Y.q.; Wang, J.; Xu, Z.h.; Zheng, W.j.; Xiao, P.g. The rediscovery of ancient Chinese herbal formulas. Phytotherapy research 2004, 18, 681-686.

9. Lu, W.I.; Lu, D.P. Impact of chinese herbal medicine on american society and health care system: perspective and concern. Evidence-Based Complementary and Alternative Medicine 2014, 2014.

10. Ang-Lee, M.K.; Moss, J.; Yuan, C.S. Herbal medicines and perioperative care. Journal of American medical association 2001, 286, 208-216.

11. Bent, S. Herbal medicine in the United States: review of efficacy, safety, and regulation. Journal of general internal medicine 2008, 23, 854-859.

12. Haller, C.A.; Benowitz, N.L. Adverse cardiovascular and central nervous system events associated with dietary supplements containing ephedra alkaloids. New England journal of medicine 2000, 343, 1833-1838.

13. U.S. Food and Drug Administration. Regulation prohibiting sale of dietary supplements containing ephedrine alkaloids and reiterates its advice that consumers stop using these products. Availabe online: http://www.fda.gov/NewsEvents/Newsroom/PressAnnouncements/2004/ucm108242.htm. (Acessed on 6 February 2004).

14. Flanagan, C.M.; Kaesberg, J.L.; Mitchell, E.S.; Ferguson, M.A.; Haigney, M.C. Coronary artery aneurysm and thrombosis following chronic ephedra use. International Journal of cardiology 2010, 139, e11-e13.

15. Abourashed, E.A.; El-Alfy, A.T.; Khan, I.A.; Walker, L. Ephedra in perspective-a current review. Phytotherapy research 2003, 17, 703-712.

16. Blechman, K.M.; Karch, S.B.; Stephens, B.G. Demographic, pathologic, and toxicological profiles of 127 decedents testing positive for ephedrine alkaloids. Forensic science international 2004, 139, 61-69.

17. Jia, J.J.; Zeng, X.S.; Li, Y.; Ma, S.; Bai, J. Ephedrine induced thioredoxin-1 expression through $\beta$-adrenergic receptor/cyclic AMP/protein kinase A/dopamine-and cyclic AMP-regulated phosphoprotein signaling pathway. Cellular signalling 2013, 25, 1194-1201.

18. Wang, J.W.; Chiang, M.H.; Lu, C.M.; Tsai, T.H. Determination the active compounds of herbal preparation by UHPLC-MS/MS and its application on the preclinical pharmacokinetics of pure ephedrine, single herbal extract of Ephedra, and a multiple herbal preparation in rats. Journal of chromatography B 2016, 1026, 152161.

19. Lee, M.; Cheng, B.; Che, C.; Hsieh, D. Cytotoxicity assessment of Ma-huang (Ephedra) under different conditions of preparation. Toxicological sciences 2000, 56, 424-430.

20. Yim, N.H.; Kim, A.; Jung, Y.P.; Kim, T.; Ma, C.J.; Ma, J.Y. Fermented So-Cheong-Ryong-Tang (FCY) induces apoptosis via the activation of caspases and the regulation of MAPK signaling pathways in cancer cells. BMC complementary and alternative medicine 2015, 15, 336.

21. Vrolijk, M.F.; Haenen, G.R.; Opperhuizen, A.; Jansen, E.H.; Schiffers, P.M.; Bast, A. The supplement-drug interaction of quercetin with tamsulosin on vasorelaxation. European journal of pharmacology 2015, 746, 132-137.

22. Zheng, F.H.; Wei, P.; Huo, H.L.; Xing, X.F.; Chen, F.L.; Tan, X.M.; Luo, J.B. Neuroprotective effect of gui zhi (ramulus cinnamomi) on ma huang-(herb ephedra-) induced toxicity in rats treated with a ma huang-gui zhi herb pair. Evidence-based complementary and alternative medicine 2015, 2015. 
23. Jin, R.; Lin, Z.J.; Xue, C.M.; Zhang, B. An improved association-mining research for exploring Chinese herbal property theory: based on data of the Shennong's Classic of Materia Medica. Journal of integrative medicine 2013, 11, 352-365.

24. Zhang, N.D.; Han, T.; Huang, B.K.; Rahman, K.; Jiang, Y.P.; Xu, H.T.; Qin, L.P.; Xin, H.L.; Zhang, Q.Y.; Li, Y.M. Traditional Chinese medicine formulas for the treatment of osteoporosis: Implication for antiosteoporotic drug discovery. Journal of ethnopharmacology 2016, 189, 61-80.

25. Kim, E.J.; Chen, Y.; Huang, J.Q.; Li, K.M.; Razmovski-Naumovski, V.; Poon, J.; Chan, K.; Roufogalis, B.D.; McLachlan, A.J.; Mo, S.L. Evidence-based toxicity evaluation and scheduling of Chinese herbal medicines. Journal of ethnopharmacology 2013, 146, 40-61.

26. He, Y.; Gai, Y.; Wu, X.; Wan, H. Quantitatively analyze composition principle of Ma Huang Tang by structural equation modeling. Journal of ethnopharmacology 2012, 143, 851-858.

27. Fei, L.; Qiaoling, F. Science of Prescriptions; Shanghai University of Traditional Chinese Medicine press: Shanghai, China, 2002.

28. Li, J.; Liu, Z.; Jiang, Y. Formulas of traditional Chinese medicine; China press of Traditional Chinese Medicine: Beijing, China, 2006.

29. Wei, P.; Huo, H.L.; Ma, Q.H.; Li, H.C.; Xing, X.F.; Tan, X.M.; Luo, J.B. Pharmacokinetic comparisons of five ephedrine alkaloids following oral administration of four different Mahuang-Guizhi herb-pair aqueous extracts ratios in rats. Journal of ethnopharmacology 2014, 155, 642-648.

30. Chao, L.K.; Hua, K.F.; Hsu, H.Y.; Cheng, S.S.; Lin, I.F.; Chen, C.J.; Chen, S.T.; Chang, S.T. Cinnamaldehyde inhibits pro-inflammatory cytokines secretion from monocytes/macrophages through suppression of intracellular signaling. Food and chemical toxicology 2008, 46, 220-231.

31. Song, S.; Chen, F.; Xing, X.; Ren, M.; Ma, Q.; Xie, Y.; Tang, Q.; Luo, J. Concurrent quantification and comparative pharmacokinetic analysis of bioactive compounds in the Herba Ephedrae-Semen Armeniacae Amarum herb pair. Journal of pharmaceutical and biomedical analysis 2015, 109, 67-73.

32. Hennell, J.R.; Lee, S.; Khoo, C.; Gray, M.J.; Bensoussan, A. The determination of glycyrrhizic acid in Glycyrrhiza uralensis Fisch. ex DC.(Zhi Gan Cao) root and the dried aqueous extract by LC-DAD. Journal of pharmaceutical and biomedical analysis 2008, 47, 494-500. 


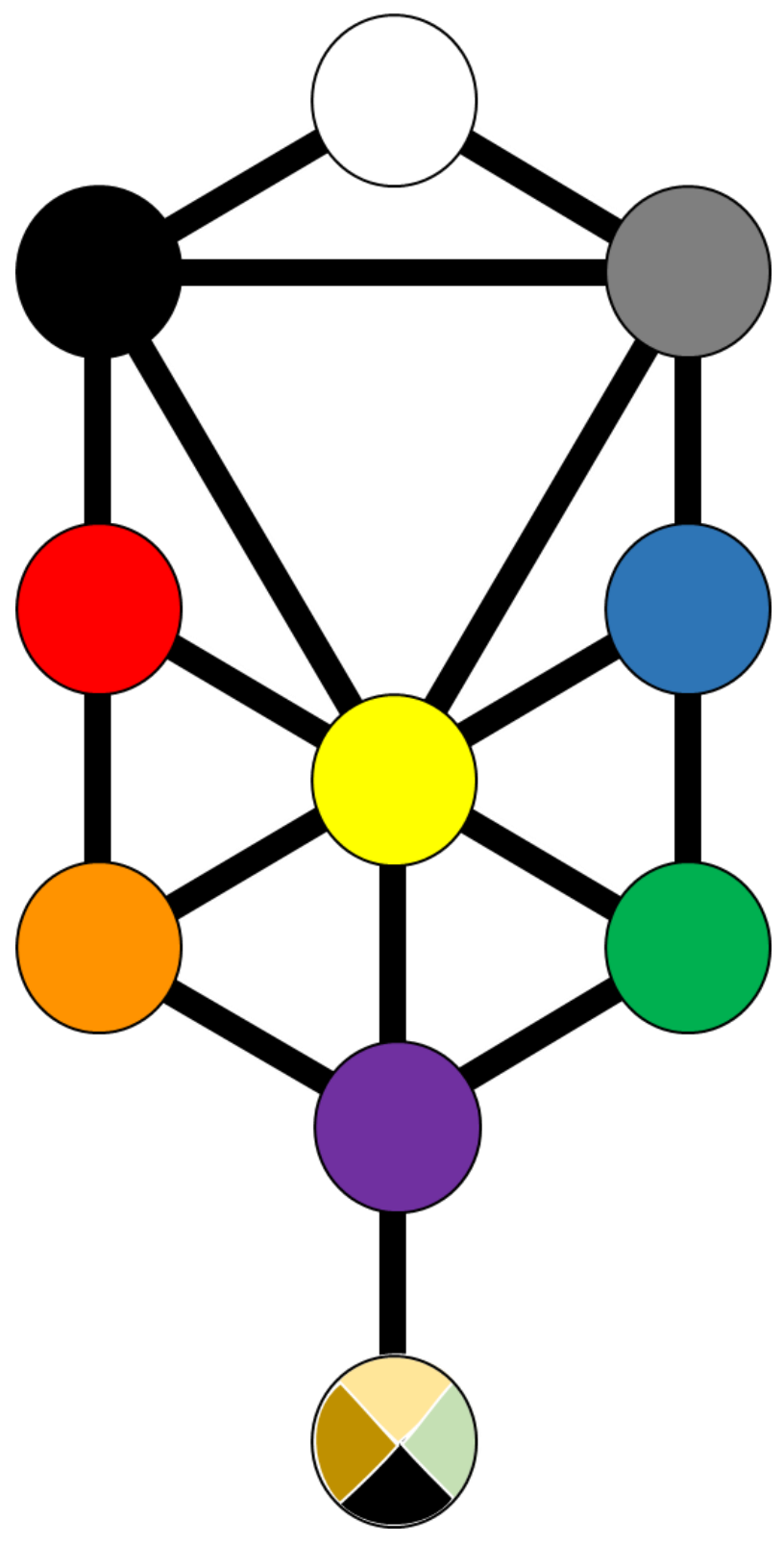




\section{Chapter 3}

\section{The screening of anticholinergic accumulation by Traditional Chinese Medicine}

Ming Zhang, Misha Vrolijk and Guido R.M.M. Haenen

International Journal of Molecular Sciences. 2017; 19(1): 18. 


\begin{abstract}
Many Western drugs can give rise to serious side effects due to their ability to bind to acetylcholine receptors in the brain. This aggravates when they are combined, which is known as anticholinergic accumulation (AA). Some bioactives in Traditional Chinese Medicine (TCM) are known to block acetylcholine receptors and thus potentially cause AA. The AA of TCM was screened by quantifying the displacement of $\left[{ }^{3} \mathrm{H}\right]$ pirenzepine on acetylcholine receptors in a rat brain homogenate. We used a new unit to express AA, namely the Total Atropine Equivalents (TOAT). The TOAT of various herbs used in TCM was very diverse and even negative for some herbs. This is indicative for the broadness of the pallet of ingredients used in TCM. Three TCM formulas were screened for AA: Ma Huang Decotion (MHD), Antiasthma Simplified Herbal Medicine intervention (ASHMI), and Yu Ping Feng San (YPFS). The TOAT of ASHMI was indicative for an additive effect of herbs used in it. Nevertheless, it can be calculated that one dose of ASHMI is probably too low to cause AA. The TOAT of YPFS was practically zero. This points to a protective interaction of AA. Remarkably, MHD gave a negative TOAT, indicating that the binding to the acetylcholine receptors was increased, which also circumvents AA. In conclusion, our results indicate that TCM is not prone to give AA and support that there is an intricate interaction between the various bioactives in TCM to cure diseases with minimal side effects.
\end{abstract}

Keywords: Traditional Chinese Medicine, anticholinergic accumulation, Total Atropine Equivalents

$$
\mathrm{TOAT}=[\mathrm{A}]=\frac{\mathrm{K}_{\mathrm{D}} *\left([\mathrm{P}] * \mathrm{Bmax}-[\mathrm{P}] * \mathrm{~B}-\mathrm{K}_{\mathrm{P}} * \mathrm{~B}\right)}{\mathrm{K}_{\mathrm{P}} * \mathrm{~B}} * \mathrm{D}
$$




\section{Introduction}

Acetylcholine receptors (AChRs), or cholinergic receptors, are important targets in pharmacotherapy. They are located in parasympathetic and sympathetic ganglia and in the brain. The muscarinic AChRs are G-protein coupled receptors and mediate a relatively slow response via second messenger cascades, while the nicotinic AChRs are ligand-gated ion channels that mediate a relatively fast response [1,2].

Many drugs have been designed to influence cholinergic transmission to obtain their therapeutic effects $[3,4]$. In addition, numerous drugs, as well as supplements, cause side effects by blocking AChRs [5]. When compounds that block AChRs are taken simultaneously, these side effects will accumulate. This interaction is known as anticholinergic accumulation (AA). AA may lead to dry mouth, blurred vison, falls, urinary disorders, impulsive behavior, and even cognitive impairment and delirium [6-8]. This is especially relevant for the elderly due to polypharmacy, changes in brain neurochemistry, and changes in pharmacokinetics and pharmacodynamics in this frail group [9-11].

Traditional Chinese Medicine (TCM) is increasingly valued in the Western world because of its effectiveness to treat diseases and its relatively mild side effects [12]. TCM is a dedicated combination of several herbs that has been developed over centuries, according to the "Jun-Chen-Zuo-Shi" principle. Within the combination, the Jun herb, main herb, is assisted by other herbs to stimulate the main pharmacological effect and to moderate side effects as well [13].

Some TCM contain bioactives that have anticholinergic activity [14]. The combination of these bioactives might aggravate this effect and cause AA. Nevertheless, TCM when used properly only results in mild side effects. This is in line with the dedicated combination of herbs in TCM. Recent studies aimed to decipher this combination principle confirmed that the interplay of the bioactives in the TCM circumvents side effects. For Western medication and supplements, the occurrence of AA is established [15]. However, there are hardly any data on the AA of TCM. This study is aimed to evaluate the potential occurrence of AA caused by TCM and to find out if AA is indeed circumvented by the dedicated combination.

To screen AA, in vitro receptor binding studies are performed. For a specific Western drug, the anticholinergic binding can be reflected by the dissociation equilibrium constant, $\mathrm{K}_{\mathrm{D}}[16,17]$. However, TCM is a mixture of compounds, and therefore it is impossible to express the anticholinergic activity of TCM by a $K_{D}$. We propose a new unit, Total Atropine Equivalents (TOAT), to estimate and express the binding of TCM toward muscarinic AChRs on the rat brain membrane. The TOAT of an herb extract is defined as the concentration of atropine that gives a binding to the muscarinic AChRs equal to that of the herbal extract. With TOAT, the AA of herbs and the combination of herbs can be accurately expressed and predicted.

\section{Materials and Methods}

\subsection{Chemicals}

$\left[{ }^{3} \mathrm{H}\right] \mathrm{PZ}(84 \mathrm{Ci} / \mathrm{mmol}$ ) was obtained from Perkin Elmer (Boston, MA, USA). Atropine, pirenzepine, cimetidine, risperidone, and theophylline were obtained from Sigma-Aldrich (St. Louis, MO, USA) and dissolved in assay buffer (50 mM potassium phosphate buffer, pH 7.4). 


\subsection{Preparation of Herb Extracts}

The Chinese herbs were supplied by Chinaturel Import \& Export B.V. (Den Haag, The Netherlands). Seven $\mathrm{mL}$ of distilled water was added to $1 \mathrm{~g}$ of the herb mixture (unless otherwise noted). After $30 \mathrm{~min}$ at room temperature, the mixture was boiled for $1 \mathrm{~h}$. The extract was centrifuged at $5000 \times g$ for $15 \mathrm{~min}$. The supernatant was filtered and stored at $20{ }^{\circ} \mathrm{C}[18]$.

Fifty diffrent herbs used to treat asthma were selected for the possibility of anticholinergic activity, since they may contain compounds that bind to AChRs. Three frequently clinical used anti-asthma herb formulas were also screened based on the potency to cause AA. They are MHD, ASHMI, and YPFS [19-21]. In each formula, all the herbs were combined in a fixed weight ratio. For MHD, this is Ma Huang (Herba Ephedra):Gui Zhi (Ramulus Cinnamomi):Xi ng Ren (Semen Armeniacae Amarum):Gan Cao (Radix Glycyrrhizae) = 3:2:2:1 [22]. For ASHMI, this is Ling Zhi (Gannoderma Lucidum):Gan Cao (Radix Glycyrrhizae):Ku Shen (Sophora Flavescens) = 20:9:3 [23]. For YPFS, this is Fang Feng (Radix Saposhnikoviae):Bai Zhu (Rhizoma Atractylodis Macrocephalae):Huang Qi (Radix Astragali) = 1:2:2 [24].

\subsection{Tissue Preparation}

Male Wistar Kyoto (WKY) rats (200-250 g; Charles River, Maastricht, The Netherlands) were sacrificed by $\mathrm{CO}_{2}$ inhalation, and brains were rapidly removed and put into the assay buffer. The brains were obtained from untreated control animals of animal studies with other objectives. These studies were conducted according to the ethical guidelines (EEC Council directives 86/609) after the animal experiments were approved by the University Animal Ethical Committee. This use of rest materials from other studies is encouraged to reduce the number of experimental animals. The homogenization of rat Brains were carried out by using a Potter tube. The Brain homogenate was first centrifuged at $500 \times g$ for $10 \mathrm{~min}$ to remove unwanted pieces. Then this homogenate was centrifuged at a higher speed at $20,000 \times g$ for $15 \mathrm{~min}$. All the centrifugations are carried out at $4{ }^{\circ} \mathrm{C}$ to protect the protein and aimed to remove all the substances that may interfere with solubility and radioligand binding assay. The final precipitated material was resuspended in assay buffer and diluted to reach the concentration of $1 \mathrm{mg}$ of protein per $0.25 \mathrm{~mL}$. The final brain homogenate was stored at $-20{ }^{\circ} \mathrm{C}$.

\subsection{Binding Assays}

For the $\left[{ }^{3} \mathrm{H}\right] \mathrm{PZ}$ binding assay, the brain homogenate was used in a concentration given above. Analysis of $\left[{ }^{3} \mathrm{H}\right] \mathrm{PZ}$ binding (final radioactive concentration: $100 \mu \mathrm{Ci} / \mathrm{mL}$ ) was determined as described by Michel et al. [25]. For saturation binding experiments, tissues were incubated in the assay buffer containing the radioligand and Chinese medicine extracts as indicated. The final assay volumes were $0.25 \mathrm{~mL}$. The binding reactions were performed at $37{ }^{\circ} \mathrm{C}$ for $60 \mathrm{~min}$ and started by addition of tissues to the mixture. After $1 \mathrm{~h}, 5$ $\mathrm{mL}$ of ice-cold assay buffer was added, followed by rapid filtration through Whatman Grade GF/C filters. Each filter was washed with an additional 2-3 $\mathrm{mL}$ of ice-cold buffer. Finally, the radioactivity of the counted filters was quantified using liquid scintillation counter in which the $\beta$ emitters $\left({ }^{3} \mathrm{H}\right)$ can be counted very effectively [15]. The amount of radioligand bound was less than $10 \%$ of total added ligand in all the experiments. 
For saturation binding experiments, tissues were incubated for $60 \mathrm{~min}$ in a concentration of $\left[{ }^{3} \mathrm{H}\right] \mathrm{PZ}(0-100 \mathrm{nM})$. Nonspecific binding was determined in parallel incubations using 1 $\mu \mathrm{M}$ atropine. Specific binding was calculated by subtracting nonspecific binding from the total binding. The affinity of atropine to muscarinic AChRs was detected after incubation of a fixed concentration of $\left[{ }^{3} \mathrm{H}\right] \mathrm{PZ}(30 \mathrm{nM})$ and different concentrations of atropine $(0.1 \mathrm{Nm}-1$ $\mu \mathrm{M})$. In the displacement experiments, tissues were carried out in the presence of $30 \mathrm{nM}$ of $\left[{ }^{3} \mathrm{H}\right] \mathrm{PZ}$ and $30 \mu \mathrm{L}$ herb extracts or $100 \mu \mathrm{M}$ drugs in a total volume of $250 \mu \mathrm{L}$. Within one hour, $\left[{ }^{3} \mathrm{H}\right] \mathrm{PZ}$ binding on muscarinic AChRs in the membrane of rat brain has reached an equilibrium. Fifty kinds of herb extracts were screened for binding to muscarinic AChRs. Moreover, herb formulas ASHMI, YPFS, and MHD were also screened for binding to muscarinic AChRs and a combination of drugs. The effect of formula MHD on the saturation binding of $\left[{ }^{3} \mathrm{H}\right] \mathrm{PZ}$ to muscarinic AChRs was also determined. All binding experiments were performed at $37{ }^{\circ} \mathrm{C}$. A control experiment showed that incubation of MHD with $\left[{ }^{3} \mathrm{H}\right] \mathrm{PZ}$ (without the brain homogenate) did not result in extra $\left[{ }^{3} \mathrm{H}\right] \mathrm{PZ}$ bound on the filter compounds to that of $\left[{ }^{3} \mathrm{H}\right] \mathrm{PZ}$ alone.

The affinity of a specific compound to muscarinic AChRs can be expressed by the dissociation constant of that compound. The herbal extracts contain many compounds, including not yet identified compounds. It is not possible to describe the affinity of a mixture of compounds by the dissociation constant. Therefore, the concentration of a reference compound (i.e., atropine) that gives a displacement that is similar to that of the herb extract is calculated. This approach is comparable to that of the Trolox Equivalent Antioxidant Capacity (TEAC). The TEAC of a mixture (e.g., a food extract or a biological sample) gives the concentration of the reference compound Trolox that has an equal antioxidant capacity as that of the mixture [26,27]. Similar to the TEAC, the TOAT can be used to discriminate between synergistic and additive effects of the compounds in the mixture.

\subsection{Calculation of the Total Atropine Equivalents (TOAT) for the Muscarinic Acetylcholine} Receptors (AChRs)

These calculations are based on the equilibrium depicted in Fig. 1 [28,29]: 


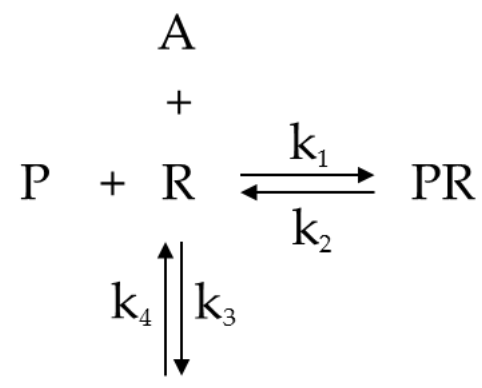

AR

Fig. 1. Competition of $\left[{ }^{3} \mathrm{H}\right] \mathrm{PZ}(\mathrm{P})$ and atropine (A) for muscarinic $\mathrm{AChRs}(\mathrm{R})$. $\mathrm{PR}$ is $\mathrm{P}$ bond to $\mathrm{R}$, and $\mathrm{AR}$ is $\mathrm{A}$ bond to $\mathrm{R} . \mathrm{k}_{1}$ and $\mathrm{k}_{2}$ are rate constants for PR association and dissociation, respectively. $\mathrm{k} 3$ and $\mathrm{k} 4$ are rate constants for AR association and dissociation, respectively.

In the equilibrium, $\mathrm{P}$ stands for $\left[{ }^{3} \mathrm{H}\right] \mathrm{PZ}, \mathrm{R}$ is the muscarinic AChRs, $\mathrm{A}$ is atropine. $\mathrm{PR}$ is $P$ bond to $R$, and $A R$ is $A$ bond to $R$. $k_{1}$ and $k_{2}$ are rate constants for $\left[{ }^{3} \mathrm{H}\right] \mathrm{PZ}$-receptor association and dissociation, respectively. $\mathrm{k}_{3}$ and $\mathrm{k}_{4}$ are rate constants for atropine-receptor association and dissociation, respectively.

The equilibrium for binding of $\mathrm{P}$ to the receptor $\mathrm{R}$ can be given by the dissociation equilibrium constant $\mathrm{K}_{\mathrm{P}}$, according to the following equation:

$$
\mathrm{K}_{\mathrm{P}}=\frac{\mathrm{k}_{2}}{\mathrm{k}_{1}}=\frac{[\mathrm{P}] *[\mathrm{R}]}{[\mathrm{PR}]}
$$

This equation can be transformed into Equation (1).

$$
[\mathrm{PR}]=\frac{[\mathrm{P}] *[\mathrm{R}]}{\mathrm{K}_{\mathrm{P}}}
$$

Similarly, the equilibrium for binding of A to the receptor is given by the dissociation equilibrium constant $\mathrm{K}_{\mathrm{D}}$ according to the following equation:

$$
\mathrm{K}_{\mathrm{D}}=\frac{\mathrm{k}_{4}}{\mathrm{k}_{3}}=\frac{[\mathrm{A}] *[\mathrm{R}]}{[\mathrm{AR}]}
$$

This equation can be transformed into Equation (2).

$$
[\mathrm{AR}]=\frac{[\mathrm{A}] *[\mathrm{R}]}{\mathrm{K}_{\mathrm{D}}}
$$


In this assay, $\left[{ }^{3} \mathrm{H}\right] \mathrm{PZ}$ is displaced by the herb extraction or drug. [A] is the concentration of atropine that has a similar displacement as herb extraction.

$B$ is the concentration of receptors bound by $\left[{ }^{3} \mathrm{H}\right] \mathrm{PZ}$ in the presence of herb extraction or drug, which is equal to [PR]. The total concentration of receptors, $\mathrm{B}_{\max }$, is the sum of $[\mathrm{R}],[\mathrm{PR}]$, and [AR].

$$
\begin{gathered}
\mathrm{B}=[\mathrm{PR}] \\
\operatorname{Bmax}=[\mathrm{R}]+[\mathrm{PR}]+[\mathrm{AR}]
\end{gathered}
$$

It can be derived that the relative amount of receptors bound by $\left[{ }^{3} \mathrm{H}\right] \mathrm{PZ}$ is

$$
\frac{\mathrm{B}}{\mathrm{Bmax}}=\frac{[\mathrm{PR}]}{[\mathrm{R}]+[\mathrm{PR}]+[\mathrm{AR}]}
$$

Substitution of Equations (1) and (2) into Equation (3) yields Equation (4).

$$
\begin{gathered}
\mathrm{B}=\frac{[\mathrm{PR}] * \mathrm{Bmax}}{[\mathrm{R}]+[\mathrm{PR}]+[\mathrm{AR}]}=\frac{\frac{[\mathrm{P}] *[\mathrm{R}]}{\mathrm{K}_{\mathrm{P}}} * \mathrm{Bmax}}{[\mathrm{R}]+\frac{[\mathrm{P}] *[\mathrm{R}]}{\mathrm{K}_{\mathrm{P}}}+\frac{[\mathrm{A}] *[\mathrm{R}]}{\mathrm{K}_{\mathrm{D}}}}=\frac{[\mathrm{AP}] * \mathrm{Bmax}}{\mathrm{K}_{\mathrm{P}}+[\mathrm{P}]+\frac{[\mathrm{A}] * \mathrm{~K}_{\mathrm{P}}}{\mathrm{K}_{\mathrm{D}}}} \\
\mathrm{B}=\frac{[\mathrm{P}] * \mathrm{~B} \max }{[\mathrm{P}]+\mathrm{K}_{\mathrm{P}}\left(1+\frac{[\mathrm{A}]}{\mathrm{K}_{\mathrm{D}}}\right)}
\end{gathered}
$$

The labelled $\left[{ }^{3} \mathrm{H}\right] \mathrm{PZ}$ binds to the receptors and atropine competes with the labelled $\left[{ }^{3} \mathrm{H}\right] \mathrm{PZ}$ for the receptors. Equation (4) can be rearranged into Equation (5).

$$
[\mathrm{A}]=\frac{\mathrm{K}_{\mathrm{D}} *\left([\mathrm{P}] * \mathrm{Bmax}-[\mathrm{P}] * \mathrm{~B}-\mathrm{K}_{\mathrm{P}} * \mathrm{~B}\right)}{\mathrm{K}_{\mathrm{P}} * \mathrm{~B}}
$$

The formula to calculate the TOAT is

$$
\mathrm{TOAT}=[\mathrm{A}]=\frac{\mathrm{K}_{\mathrm{D}} *\left([\mathrm{P}] * \mathrm{Bmax}-[\mathrm{P}] * \mathrm{~B}-\mathrm{K}_{\mathrm{P}} * \mathrm{~B}\right)}{\mathrm{K}_{\mathrm{P}} * \mathrm{~B}} * \mathrm{D}
$$


In this equation, $\mathrm{D}$ is a factor of 8.33 that accounts for the dilution in the assay, in which $30 \mu \mathrm{L}$ of herb extract was used in a total volume of $250 \mu \mathrm{L}$. To determine the TOAT, we used a $K_{D}$ of atropine to muscarinic AChRs of $9.34 \times 10^{-9} \mathrm{M}$ and a $K_{P}$ of $\left[{ }^{3} \mathrm{H}\right] \mathrm{PZ}$ to muscarinic AChRs of $1.40 \times 10^{-9} \mathrm{M}$. Both were determined experimentally and comparable to the value found in literature. The dimension of the TOAT of the herb extract determined according to this procedure is $n M$.

$\mathrm{B}_{\max }$ was determined from the specific binding of $\left[{ }^{3} \mathrm{H}\right] \mathrm{PZ}$ to the receptors without the tested compounds. The formula is

$$
\operatorname{Bmax}=\frac{\mathrm{B} *\left([\mathrm{P}]+\mathrm{K}_{\mathrm{P}}\right)}{[\mathrm{P}]}
$$

$\mathrm{B}^{*}$ is the specific binding of $\left[{ }^{3} \mathrm{H}\right] \mathrm{PZ}$ to the receptors without tested compounds.

The TOAT of a drug was determined by the displacement of $\left[{ }^{3} \mathrm{H}\right] \mathrm{PZ}$ by a $100 \mu \mathrm{M}$ solution of the drugs. The concentration of atropine that gave the same displacement as 100 $\mu \mathrm{M}$ (final concentration) of the drug was calculated according to Equation (6), with $\mathrm{D}=1$. The TOAT of the drug reflects the potency of drug to displace $\left[{ }^{3} \mathrm{H}\right] \mathrm{PZ}$ relative to that of atropine on a molar basis. Therefore the dimension of the TOAT of a drug obtained in this way is $10^{-5}$ (mol atropine)/(mol drug).

\subsection{Statistics}

Data are shown as mean \pm standard error of the mean (S.E.M.). $n$ refers to the number of independent experiments that repeated. Data were analyzed in Prism 5 (GraphPad Software Inc., La Jolla, CA, USA). The data of total binding, nonspecific and specific binding were fitted to a nonlinear regression curve-one binding site. The kinetic parameters, $\mathrm{K}_{\mathrm{D}}$ of $\left[{ }^{3} \mathrm{H}\right] \mathrm{PZ}$ and $\mathrm{B}_{\max }$, were calculated by fitting to one site-specific binding. The results of atropine displacement experiments were fitted to one site binding competitive (fitting $\mathrm{K}_{\mathrm{i}}$ ) to calculated $\mathrm{K}_{\mathrm{D}}$. Data were analyzed using a two-tailed Student's $t$-test. $p$-value $<0.05$ were considered statistically significant.

\section{Results}

In Fig. 1, The binding of $\left[{ }^{3} \mathrm{H}\right]$ pirenzepine $\left(\left[{ }^{3} \mathrm{H}\right] \mathrm{PZ}\right)$ to muscarinic AChRs in rat brains is depicted. Non-specific binding was determined by incubating brain homogenate with a 100 $\mu \mathrm{M}$ atropine and various concentrations of $\left[{ }^{3} \mathrm{H}\right] \mathrm{PZ}$ over the range 1 to $100 \mathrm{nM}$. The $\mathrm{K}_{\mathrm{P}}$ of the binding of $\left[{ }^{3} \mathrm{H}\right] \mathrm{PZ}$ to the AChRs was $9.3 \times 10^{-9} \mathrm{M}$, and the concentration of muscarinic AChRs $\left(\mathrm{B}_{\max }\right)$ in the rat brain was $18.0 \pm 1.3 \mathrm{fmol} / \mathrm{mg}$ protein (Fig. $2 \mathrm{~A}$ ). A $\mathrm{K}_{\mathrm{D}}$ of atropine for the AChRs found in the displacement experiment was $1.4 \times 10^{-9} \mathrm{M}$ (Fig. 2B). 


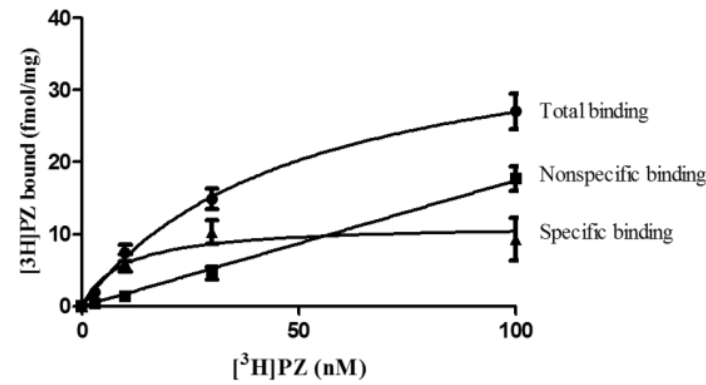

(A)

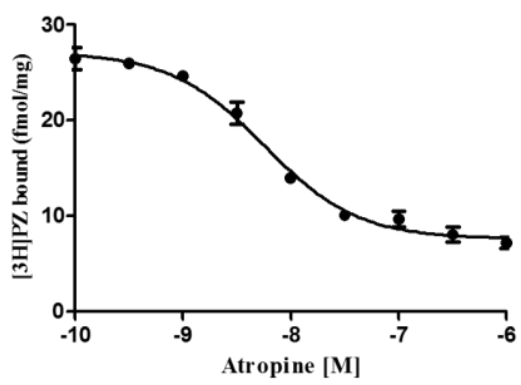

(B)

Fig. 2. (A) Total, nonspecific and specific binding of $\left[{ }^{3} \mathrm{H}\right]$ pirenzepine $\left(\left[{ }^{3} \mathrm{H}\right] \mathrm{PZ}\right)$ to muscarinic acetylcholine receptors (AChRs) in the rat brain homogenate. For the nonspecific binding, $10^{-6} \mathrm{M}$ atropine was added to displace $\left[{ }^{3} \mathrm{H}\right] \mathrm{PZ}$ from the AChRs. The $\mathrm{K}_{\mathrm{P}}$ of $\left[{ }^{3} \mathrm{H}\right] \mathrm{PZ}$ found was $9.3 \times 10^{-9} \mathrm{M}$; $(\mathbf{B})$ Displacement of $\left[{ }^{3} \mathrm{H}\right] \mathrm{PZ}(30 \mathrm{nM})$ to muscarinic AChRs by atropine. The $\mathrm{K}_{\mathrm{D}}$ of atropine found was $1.4 \times 10^{-9} \mathrm{M}$. Data are shown as mean \pm standard error of the mean (S.E.M.) $(n=3)$.

To determine the binding of Western drugs and TCM to muscarinic AChRs, in vitro receptor binding studies are used. The TOAT of cimetidine, risperidone and theophylline were 4,6 and $1 \times 10^{-5} \mathrm{~mol}$ atropine/mol drug, respectively. The TOAT of the combination found experimentally was $11 \times 10^{-5} \mathrm{~mol}$ atropine/mol drug, which is equal to the sum of the TOAT of the individual drugs (Fig. 3). That the experimental TOAT equaled to the calculated TOAT, indicates that the effects of these Western drugs are additive and prone to cause AA.

\section{Western drugs}

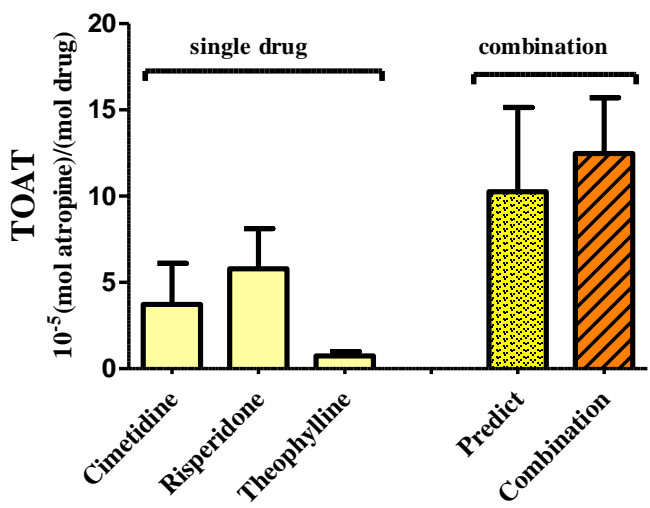

Fig. 3. The Total Atropine Equivalents (TOAT) of the Western drugs cimetidine, risperidone, and theophylline compared to the TOAT of the combination of these Western drugs. The TOAT calculated by summing the TOAT of the single drugs was similar to the experimental TOAT. This indicated that the effects are additive. 
Fifty different herbs used in TCM were screened for the binding to muscarinic AChRs and expressed as the TOAT (Fig. 4). A high TOAT indicates that this herb can displace $\left[{ }^{3} \mathrm{H}\right] \mathrm{PZ}$ effectively, whereas a low TOAT indicates relatively less displacement. The values of TOAT covered a wide range. Interestingly, some herbs had a negative TOAT, which means that these herbs increase the binding of $\left[{ }^{3} \mathrm{H}\right] \mathrm{PZ}$.

TCM usually consists of a mixture of herbs. The TOATs of the TCM formulas and that of the individual herbs combined in the TCM formulas were determined (Fig. 5). Similar to the combination of Western drugs, the experimental TOAT value of the TCM formula Antiasthma Simplified Herbal Medicine intervention (ASHMI), is equal to the sum of the TOAT of the individual herbs. That the experimental TOAT equaled the calculated TOAT indicates that the effects of the herbs in ASHMI are additive. In the formula Yu Ping Feng San (YPFS), the experimental TOAT is much lower than the calculated TOAT, which indicates that the blocking to receptors is decreased by using this combination. This would protect against AA. Remarkably, the formula Ma Huang Decotion (MHD) gives a negative TOAT, indicating that the binding to muscarinic AChRs was increased. This also points to a protection against AA by TCM.

To further investigate the increase in $\left[{ }^{3} \mathrm{H}\right] \mathrm{PZ}$ binding by MHD, we tested the effect of MHD on binding of various concentration of $\left[{ }^{3} \mathrm{H}\right] \mathrm{PZ}$ to muscarinic AChRs. It was found that addition of MHD dose dependently increased the binding of $\left[{ }^{3} \mathrm{H}\right] \mathrm{PZ}$ to AChRs over the entire concentration range tested (Fig. 6). 


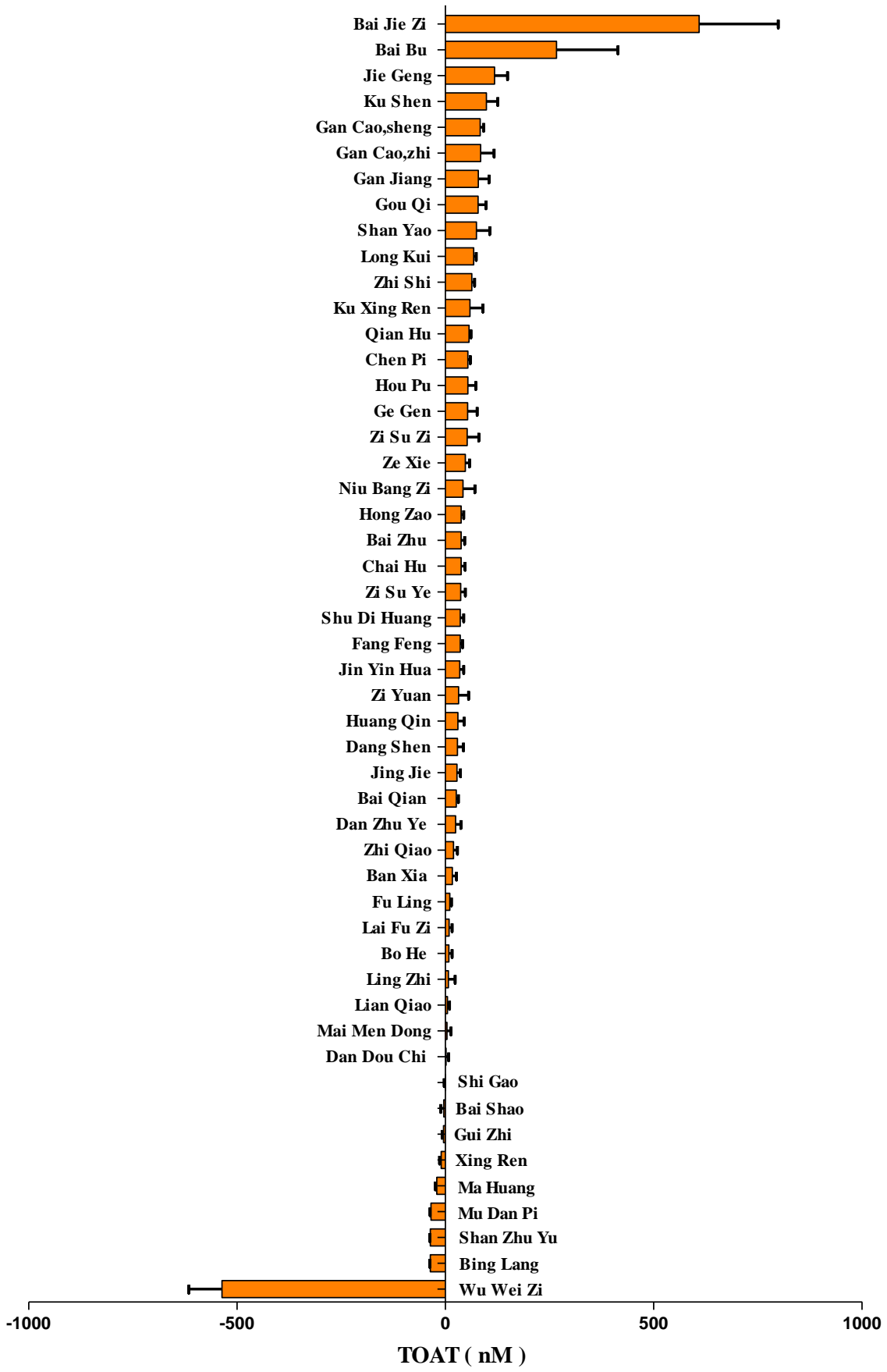

Fig. 4. The TOAT of herbs used in Traditional Chinese Medicine (TCM). The TOAT of 50 herbs used in TCM. Data are shown as mean \pm S.E.M. $(n=3)$. 

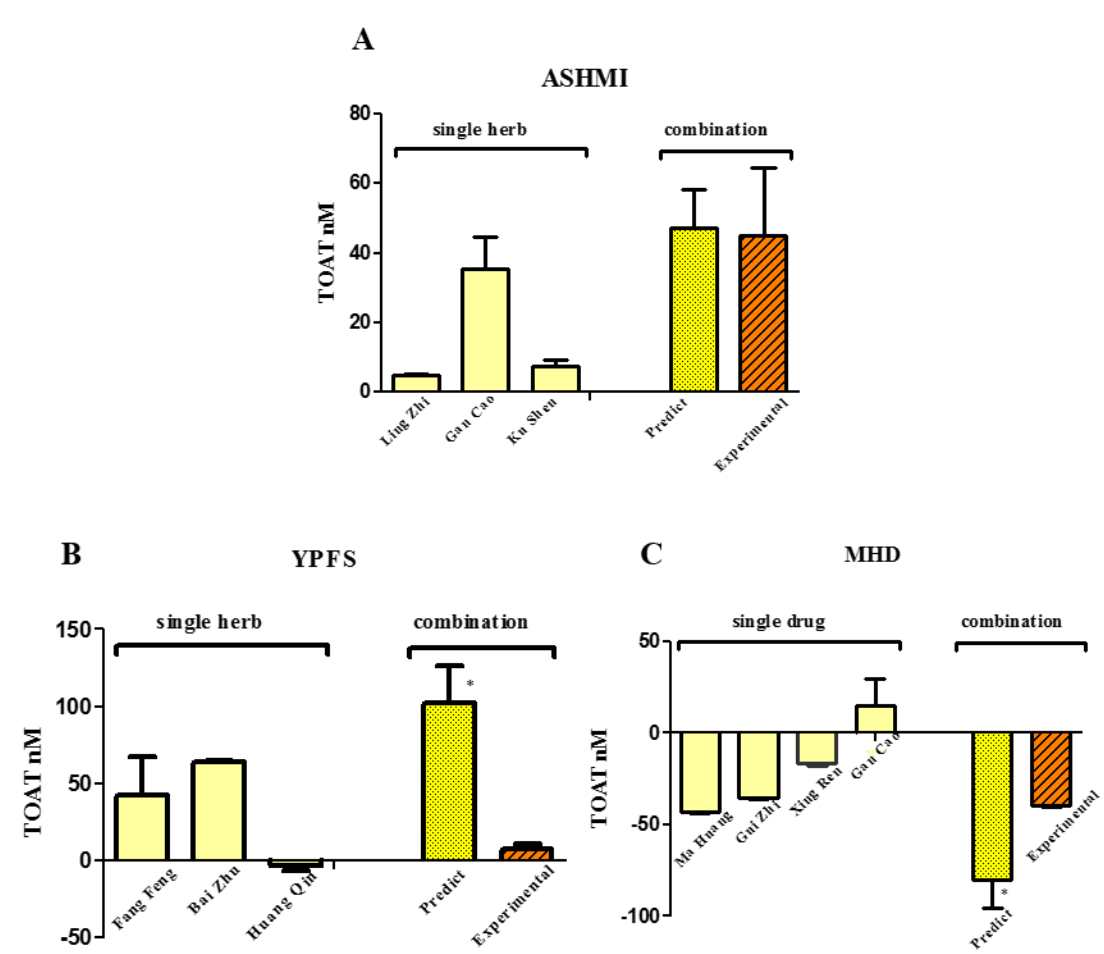

Fig. 5. The TOAT of three different TCM formulas, and the TOAT of herbs used in the formulas. (A) Ma Huang Decotion (MHD), (B) Antiasthma Simplified Herbal Medicine intervention (ASHMI), (C) Yu Ping Feng San (YPFS). The predicted TOAT was calculated by summing the TOATs of the herbs. Predicted TOAT significantly different from the experimental TOAT $(* p<0.05)$.

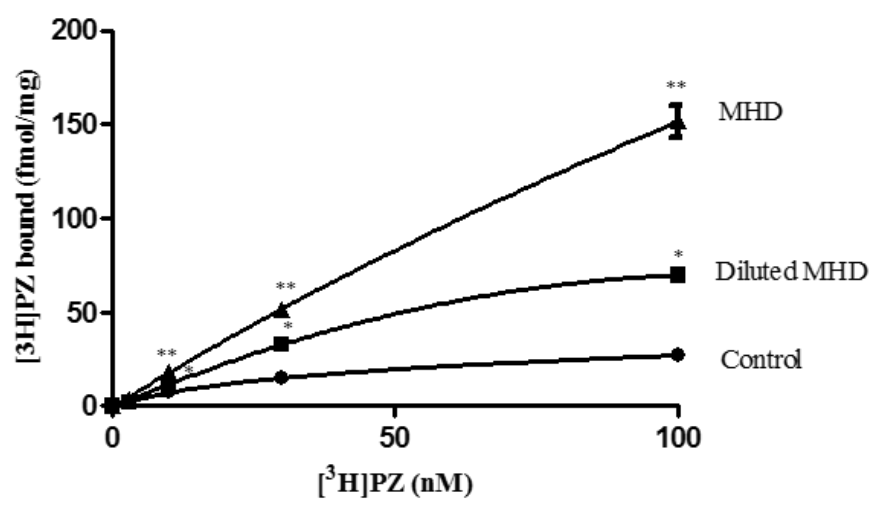

Fig. 6. Total binding of $\left[{ }^{3} \mathrm{H}\right] \mathrm{PZ}$ to muscarinic AChRs without and with $30 \mu \mathrm{L} \mathrm{MHD}$, and $30 \mu \mathrm{L}$ of $1: 5$ diluted MHD. MHD dose independently increase the binding of $\left[{ }^{3} \mathrm{H}\right] \mathrm{PZ}$. ** Significantly different from control and MHD. * Significantly different from control and diluted MHD. 


\section{Discussion}

Compared with the relatively young Western drugs, TCM has matured over centuries. In TCM, several herbs are combined to treat a disease. When used properly, the side effects of TCM are relatively mild which is attributed to the intricate interplay of the various bioactives in the delicately assembled combination of herbs according to the "Jun-ChenZuo-Shi" principle [13]. This absence of major side effects is one of the reasons that TCM is increasingly valued in the Western societies. With regard to anticholinergic side effects, it should be noted that some herbs used in TCM are expected to display anticholinergic activity since they contain bioactives that are known to block AChRs [30]. The combination of these herbs, as is done in TCM, might even lead to AA. The aim of the present study was to determine the binding of TCM to AChRs to screen the potential risk of AA of TCM.

AA was screened in a receptor binding assay. We used a new unit, TOAT, to estimate and express the binding of TCM towards muscarinic AChRs. The TOAT is defined as the concentration of atropine that gives an equal displacement as that of an herbal extract. A relatively high TOAT means a higher concentration of atropine and is predictive for an increased risk of anticholinergic adverse effects. The TOAT can also be used to evaluate the interaction between different herbs.

The TOATs of cimetidine, risperidone, and theophylline were relatively small, and the combination of these three drugs results in a substantial TOAT value that was equal to the sum of individual TOATs of the drugs. This is indicative for an additive interaction of these drugs and for the potential occurrence of AA with their combination. Similar results were reported by Vrolijk et al., in which some drugs or supplements showed an additive interaction for AA when they were combined [15].

We screened 50 different herbs used in TCM for their anticholinergic activity. It appeared that the potency to displace the radiolabel from the AChRs greatly differed between the herb extracts. Some showed a relatively high TOAT value, indicative of their potency to block AChRs. Interestingly, some herb extracts had a negative TOAT value, meaning that the bioactives in these herbs increase the binding of the radiolabel to the AChRs. This high variation of the TOAT between the herb extracts demonstrates the broadness of the pallet of bioactives used in TCM.

We also screened some TCM formulas and compared them to the individual herbs they consist of. In herb formula ASHMI, the experimental TOAT value is similar to the TOAT calculated by summing the TOAT values of the individual herbs. That the experimental TOAT equals the calculated TOAT suggests that the effects are additive and indicates a risk of AA of the combination of these herbs. However, it should be noted that despite the additive interaction between the herbs, the TOAT was still relatively low. It can be calculated that $400 \mathrm{~mL}$ (the normal dose) of ASHMI that has a TOAT of $40 \mathrm{nM}$, is equal to a dose of $0.005 \mathrm{mg}$ atropine, which is much lower than the therapeutic dose of atropine (1-2 $\mathrm{mg}$ ). This indicates the ASHMI is not likely to give AA. For comparison, using the TOAT of cimetidine, which is $4 \times 10^{-5} \mathrm{~mol}$ atropine/mol cimetidine, a regular dose of $800 \mathrm{mg}$ cimetidine equals $0.032 \mathrm{mg}$ atropine. This is 6 times more than the $0.005 \mathrm{mg}$ of atropine equivalents of ASHMI, indicating that the risk with ASHMI is even lower than the relatively low risk of AA with cimetidine alone. Apparently, the anticholinergic effect of the bioactives in ASHMI is too low to give rise to AA. This is in line with the concept that TCM has a "soft" effect and not a "hard" effect like Western drugs. 
Interestingly, in the herb formula YPFS, the experimental TOAT value is less than the calculated TOAT value. This implies that the blocking of AChRs is less than expected, and this is indicative for a protection against AA by this formula. This reveals the intricate interaction between the bioactives in TCM that is used to cure diseases with minimal side effects. This corroborates the general accepted image of TCM, that when used properly, TCM does not give rise to serious side effects. However, clinical studies on the AA of TCM is lacking at this moment, so according to Western standards, this is not "evidence based".

The most striking finding of our study is that the TOAT of some herbs and formula MHD is negative. This means that the binding of the radiolabel is increased by adding compounds that were expected to reduce the binding. This does not fit in the generally accepted theory on receptor binding. Nevertheless, several phenomena observed in Western pharmacology might explain the increased binding observed with TCM. One is that the 3D structure of the receptor protein is affected by the bioactives in the TCM. The bioactives might bind to sites that induce a conformational change of the binding site that promotes binding [31]. Moreover, receptors are known to dimerize, and this might also be affected. It has been proved that heterodimerization can increase or decrease ligand binding and change the cellular response to receptor activation. Dimerization of receptors can also increase ligand binding [32]. In addition, the microenvironment of the receptor might be affected by TCM. Lipid rafts and caveolae regulate receptor activity at all the stages of their lifecycle [33]. By changing the microenvironment, TCM might also modulate the binding characteristics of the receptors.

We also tested the total binding of $\left[{ }^{3} \mathrm{H}\right] \mathrm{PZ}$ to muscarinic AChRs together with MHD, which has a negative TOAT. It was shown that the binding of $\left[{ }^{3} \mathrm{H}\right] \mathrm{PZ}$ was greatly increased. The extraordinary effects of TCM in the relatively simple receptor binding assay show that we only partially understand the intricate interplay of the bioactives in TCM.

\section{Conclusions}

In conclusion, a radioligand binding assay interpreted using the TOAT seems a convenient way to screen for AA of TCM. Our results indicate that when used properly, TCM is not prone to give AA. The results also show that the effects of TCM on radioligand binding are complex and that contra intuitive interactions occur. This demonstrates the broadness of pallet of the bioactives as well as their intricate interplay in TCM, which is designed to cure diseases with minimal side effects. Western pharmacology might benefit from further unraveling of these interactions, which will also help to fully appreciate TCM in the West. 


\section{References}

1. Pueves, R.D. Function of muscarinic and nicotinic acetylcholine receptors. Nature 1976, 261, 149.

2. Bishara, D.; Harwood, D.; Sauer, J.; Taylor, D.M. Anticholinergic effect on cognition (AEC) of drugs commonly used in older people. International journal of geriatric psychiatry 2017, 32, 650-656.

3. Campbell, N.; Boustani, M.; Limbil, T.; Ott, C.; Fox, C.; Maidment, I.; Schubert, C.C.; Munger, S.; Fick, D.; Miller, D. The cognitive impact of anticholinergics: a clinical review. Clinical interventions in aging 2009, 4, 225 .

4. Boustani, M.; Campbell, N.; Munger, S.; Maidment, I.; Fox, C. Impact of anticholinergics on the aging brain: a review and practical application. 2008.

5. Kalisch Ellett, L.M.; Pratt, N.L.; Ramsay, E.N.; Barratt, J.D.; Roughead, E.E. Multiple anticholinergic medication use and risk of hospital admission for confusion or dementia. Journal of the American geriatrics society 2014, 62, 1916-1922.

6. Han, L.; Agostini, J.V.; Allore, H.G. Cumulative anticholinergic exposure is associated with poor memory and executive function in older men. Journal of the American geriatrics society 2008, 56, 2203-2210.

7. Collamati, A.; Martone, A.M.; Poscia, A.; Brandi, V.; Celi, M.; Marzetti, E.; Cherubini, A.; Landi, F. Anticholinergic drugs and negative outcomes in the older population: from biological plausibility to clinical evidence. Aging clinical and experimental research 2016, 28, 25-35.

8. Rudolph, J.L.; Salow, M.J.; Angelini, M.C.; McGlinchey, R.E. The anticholinergic risk scale and anticholinergic adverse effects in older persons. Archives of internal medicine 2008, 168, 508-513.

9. Salahudeen, M.S.; Duffull, S.B.; Nishtala, P.S. Anticholinergic burden quantified by anticholinergic risk scales and adverse outcomes in older people: a systematic review. BMC geriatrics 2015, 15, 31 .

10. Mintzer, J.; Burns, A. Anticholinergic side-effects of drugs in elderly people. Journal of the royal society of medicine 2000, 93, 457-462.

11. Durán, C.E.; Azermai, M.; Vander Stichele, R.H. Systematic review of anticholinergic risk scales in older adults. European journal of clinical pharmacology 2013, 69, 1485-1496.

12. Jin, R.; Lin, Z.J.; Xue, C.M.; Zhang, B. An improved association-mining research for exploring Chinese herbal property theory: based on data of the Shennong's Classic of Materia Medica. Journal of integrative medicine 2013, 11, 352-365.

13. Zhang, N.D.; Han, T.; Huang, B.K.; Rahman, K.; Jiang, Y.P.; Xu, H.T.; Qin, L.P.; Xin, H.L.; Zhang, Q.Y.; Li, Y.M. Traditional Chinese medicine formulas for the treatment of osteoporosis: Implication for antiosteoporotic drug discovery. Journal of ethnopharmacology 2016, 189, 61-80.

14. Cheng, K.L.; Chan, Y.C.; Mak, T.; Tes, M.L.; Lau, F.L. Chinese herbal medicine-induced anticholinergic poisoning in Hong Kong. Hong Kong medical journal 2013, 19, 38-41.

15. Vrolijk, M.F.; Opperhuizen, A.; Jansen, E.H.; Bast, A.; Haenen, G.R. Anticholinergic accumulation: A slumbering interaction between drugs and food supplements. Basic \& clinical pharmacology \& toxicology 2015, 117, 427-432.

16. Chew, M.L.; Mulsant, B.H.; Pollock, B.G.; Lehman, M.E.; Greenspan, A.; Kirshner, M.A.; Bies, R.R.; Kapur, S.; Gharabawi, G. A model of anticholinergic activity of atypical antipsychotic medications. Schizophrenia research 2006, 88, 63-72.

17. Yung-Chi, C.; Prusoff, W.H. Relationship between the inhibition constant $\left(\mathrm{K}_{\mathrm{i}}\right)$ and the concentration of inhibitor which causes 50 percent inhibition $\left(\mathrm{I}_{50}\right)$ of an enzymatic reaction. Biochemical pharmacology 1973, 22, 3099-3108.

18. Lee, M.; Cheng, B.; Che, C.; Hsieh, D. Cytotoxicity assessment of Ma-huang (Ephedra) under different conditions of preparation. Toxicological sciences 2000, 56, 424-430.

19. Li, X.M.; Brown, L. Efficacy and mechanisms of action of traditional Chinese medicines for treating asthma and allergy. Journal of allergy and clinical immunology 2009, 123, 297-306.

20. He, Y.; Gai, Y.; Wu, X.; Wan, H. Quantitatively analyze composition principle of Ma Huang Tang by structural equation modeling. Journal of ethnopharmacology 2012, 143, 851-858.

21. Huang, T.P.; Liu, P.H.; Lien, A.Y.; Yang, S.L.; Chang, H.H.; Yen, H.R. Characteristics of traditional Chinese medicine use in children with asthma: a nationwide population-based study. Allergy 2013, 68, 1610-1613.

22. Yao, Y.; Zhang, X.; Wang, Z.; Zheng, C.; Li, P.; Huang, C.; Tao, W.; Xiao, W.; Wang, Y.; Huang, L. Deciphering the combination principles of Traditional Chinese Medicine from a systems pharmacology perspective based on Ma-huang Decoction. Journal of ethnopharmacology 2013, 150, 619-638.

23. Liu, C.; Yang, N.; Song, Y.; Wang, L.; Zi, J.; Zhang, S.; Dunkin, D.; Busse, P.; Weir, D.; Tversky, J. Ganoderic acid C1 isolated from the anti-asthma formula, ASHMITM suppresses TNF- $\alpha$ production by mouse macrophages and peripheral blood mononuclear cells from asthma patients. International immunopharmacology 2015, 27, 224-231. 
24. Li, T.; Wang, Y.; Wang, Y.; Liang, R.; Zhang, D.; Zhang, H.; Chen, L.; Yang, W. Development of an SPEHPLC-MS method for simultaneous determination and pharmacokinetic study of bioactive constituents of Yu Ping Feng San in rat plasma after oral administration. Journal of ethnopharmacology 2013, 145, 784792.

25. Michel, M.C.; Büscher, R.; Philipp, T.; Brodde, O.E. $\alpha 1 \mathrm{~A}$ and $\alpha$ 1B-adrenoceptors enhance inositol phosphate generation in rat renal cortex. Naunyn-Schmiedeberg's archives of pharmacology 1993, 347, 180185.

26. Fischer, M.A.; Gransier, T.J.; Beckers, L.M.; Bekers, O.; Bast, A.; Haenen, G.R. Determination of the antioxidant capacity in blood. Clinical chemistry and laboratory medicine 2005, 43, 735-740.

27. van den Berg, R.; Haenen, G.R.; van den Berg, H.; Bast, A. Applicability of an improved Trolox equivalent antioxidant capacity (TEAC) assay for evaluation of antioxidant capacity measurements of mixtures. Food chemistry 1999, 66, 511-517.

28. Haenen, G.R. Thiols in oxidative stress: Some implications for catecholamine toxicity. Doctoral thesis, Vrije University, Amsterdam, the Netheralnds, 1989.

29. Cheng, H.C. The power issue: Determination of $\mathrm{K}_{\mathrm{B}}$ or $\mathrm{K}_{\mathrm{i}}$ from $\mathrm{IC}_{50}$ : A closer look at the Cheng-Prusoff equation, the Schild plot and related power equations. Journal of pharmacological and toxicological methods 2001, 46, 61-71.

30. Chan, T. Anticholinergic poisoning due to Chinese herbal medicines. Veterinary and human toxicology 1995, 37, 156-157.

31. Chou, K.C. Insights from modelling the 3D structure of the extracellular domain of $\alpha 7$ nicotinic acetylcholine receptor. Biochemical and biophysical research communications 2004, 319, 433-438.

32. Terrillon, S.; Bouvier, M. Roles of G-protein-coupled receptor dimerization: From ontogeny to signalling regulation. EMBO reports 2004, 5, 30-34.

33. Chini, B.; Parenti, M. G-protein coupled receptors in lipid rafts and caveolae: how, when and why do they go there? Journal of molecular endocrinology 2004, 32, 325-338. 

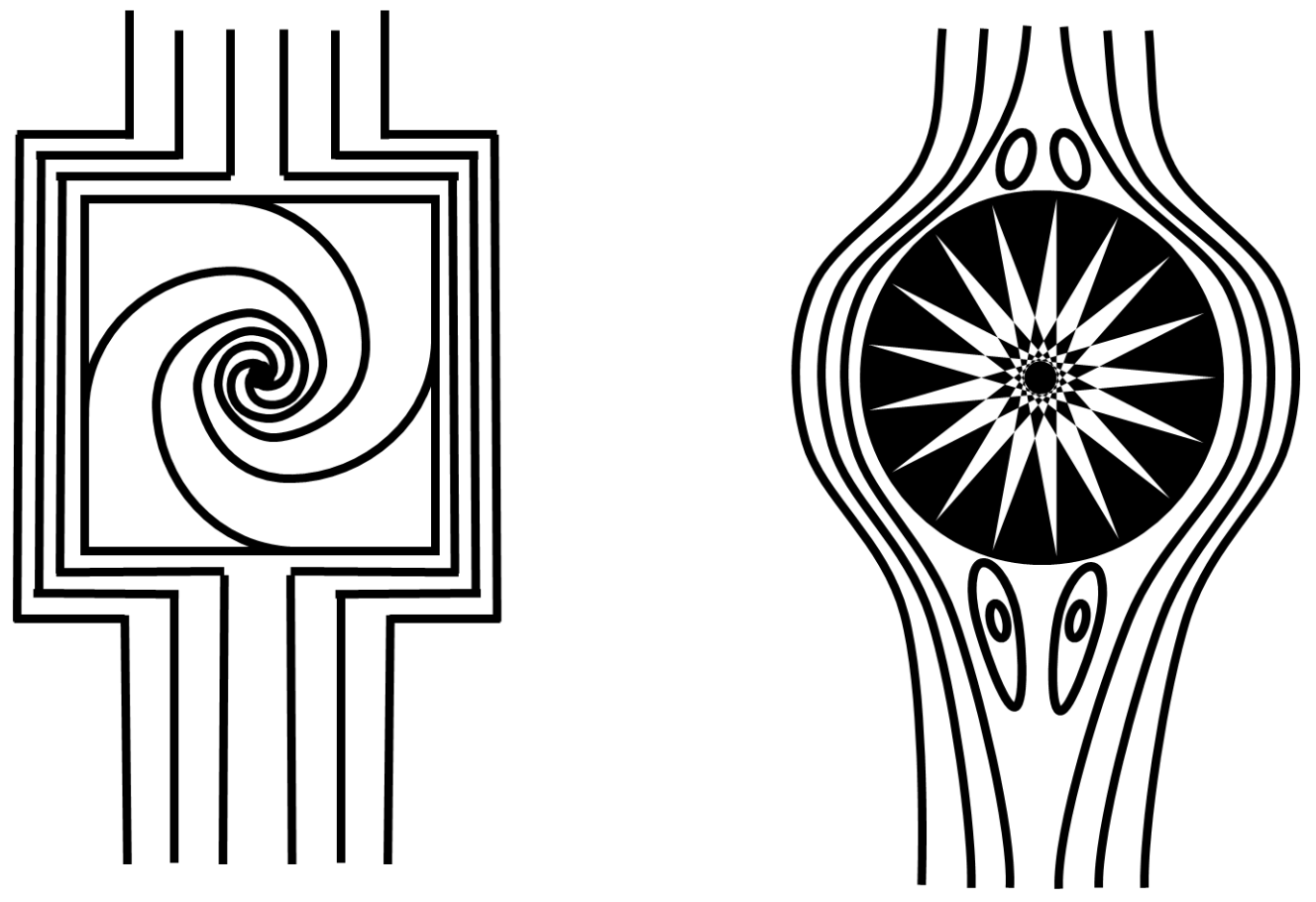



\section{Chapter 4}

\section{The chemical reactivity of (-)-epicatechin quinone mainly resides in its B-ring}

Ming Zhang, Lily Vervoort, Mohamed Moalin, Alex Mommers, Caroline Douny, Gertjan J.M. den Hartog and Guido R.M.M.

Haenen

Free Radial Biological and Medicine. 2018; 124: 31-39. 


\begin{abstract}
As one of the important dietary antioxidants, (-)-epicatechin is a potent reactive oxygen species (ROS) scavenger involved in the redox modulation of the cell. When scavenging ROS, (-)-epicatechin will donate two electrons and become (-)-epicatechin quinone, and thus take over part of the oxidative potential of the ROS. The aim of the study is to determine where this chemical reactivity resides in (-)-epicatechin quinone. When this reactivity is spread out over the entire molecule, i.e. over the AC-ring and B-ring, this will lead to partial epimerization of (-)-epicatechin quinone to (-)-catechin quinone. In our experiments, (-)-epicatechin quinone was generated with tyrosinase. The formation of (-)epicatechin quinone was confirmed by trapping with GSH, and identification of (-)epicatechin-GSH adducts. Moreover, (-)-epicatechin quinone could be detected using QTOF/MS despite its short half-life. To detect the epimerization, the ability of ascorbate to reduce the unstable flavonoid quinones into the corresponding stable flavonoids was used. The results showed that the reduction of the formed (-)-epicatechin quinone by ascorbate did not result in the formation of an appreciable amount of (-)-catechin. Therefore it can be concluded that the chemical reactivity of (-)-epicatechin quinone mainly resides in its Bring. This could be corroborated by quantum chemical calculations. Understanding the stabilization of the (-)-epicatechin quinone will help to differentiate between flavonoids and to select the appropriate compound for a specific disorder.
\end{abstract}

Keywords: (-)-epicatechin, (-)-catechin, antioxidant, quinone, epimerization

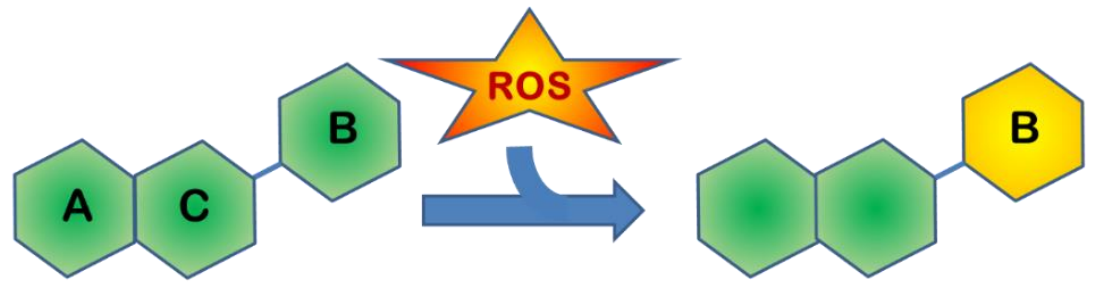

(-)-Epicatechin

(-)-Epicatechin quinone 


\section{Introduction}

Reactive oxygen species (ROS) are byproducts of oxygen metabolism in our body and can damage critical molecules in a cell $[1,2]$. To protect DNA, proteins, lipids and other vital cellular constituents against ROS damage, our cells are equipped with an intricate antioxidant defense system. However, when ROS production outbalances the defense system, the resulting oxidative stress will induce cell damage. This is a process pivotal in numerous diseases like cardiovascular diseases, arthritis, cancer, chronic inflammation, diabetes, aging, neurological and neurodegenerative disorders [3-5]. Many studies have proved that dietary antioxidants support the defense system in dealing with oxidative stress, and the intake of dietary antioxidants is associated with a lower risk of oxidative stress induced diseases [6,7].

Polyphenols form a major group of dietary antioxidants. For several decades, they have been in the spotlight because of their potent antioxidant activity, which is linked to their anti-proliferative, anti-diabetic, anticancer, anti-microbial, anti-inflammatory and antiviral activity [8,9]. During oxidative stress, the polyphenols quickly react with the ROS [10,11]. In this direct scavenging reaction, the polyphenols donate electrons to ROS, and oxidation products, quinones, are generated [12-14]. Although relatively stable compared to ROS, these quinones have taken over part of the oxidative potential of the ROS. For example, they can react with thiols present in glutathione (GSH) and cellular proteins [15-17].

In the present study, we selected one of the most important polyphenolic compounds, i.e. (-)-epicatechin $[18,19]$. The aim is to determine where the chemical reactivity resides in the (-)-epicatechin quinone. Based on its chemical structure, (-)-epicatechin quinone might be stabilized by the tautomerization as depicted in Fig. 1. This means that the reactivity is spread over the AC-ring and B-ring. If the proposed tautomerization takes place, this will lead to the partial epimerization of (-)-epicatechin quinone to (-)-catechin quinone. In the antioxidant network, the relatively unstable flavonoid quinones can be recycled by ascorbate to the corresponding stable flavonoids. Therefore oxidation of (-)-epicatechin, followed by the epimerization and the reduction of the (-)-epicatechin quinone by ascorbate, will lead to the formation of (-)-catechin out of (-)-epicatechin. We determined the extent of epimerization of (-)-epicatechin to (-)-catechin during this oxidation / reduction cycle. This helps to understand where the oxidative potential of (-)-epicatechin quinone resides within the molecule. Understanding the stabilization of the quinones will help us to differentiate between polyphenolics and to select the appropriate compound for a specific disorder. Moreover, the epimerization of (-)-epicatechin to (-)-catechin would be a tool to evaluate the antioxidant activity and recycling of (-)-epicatechin in biological systems. 


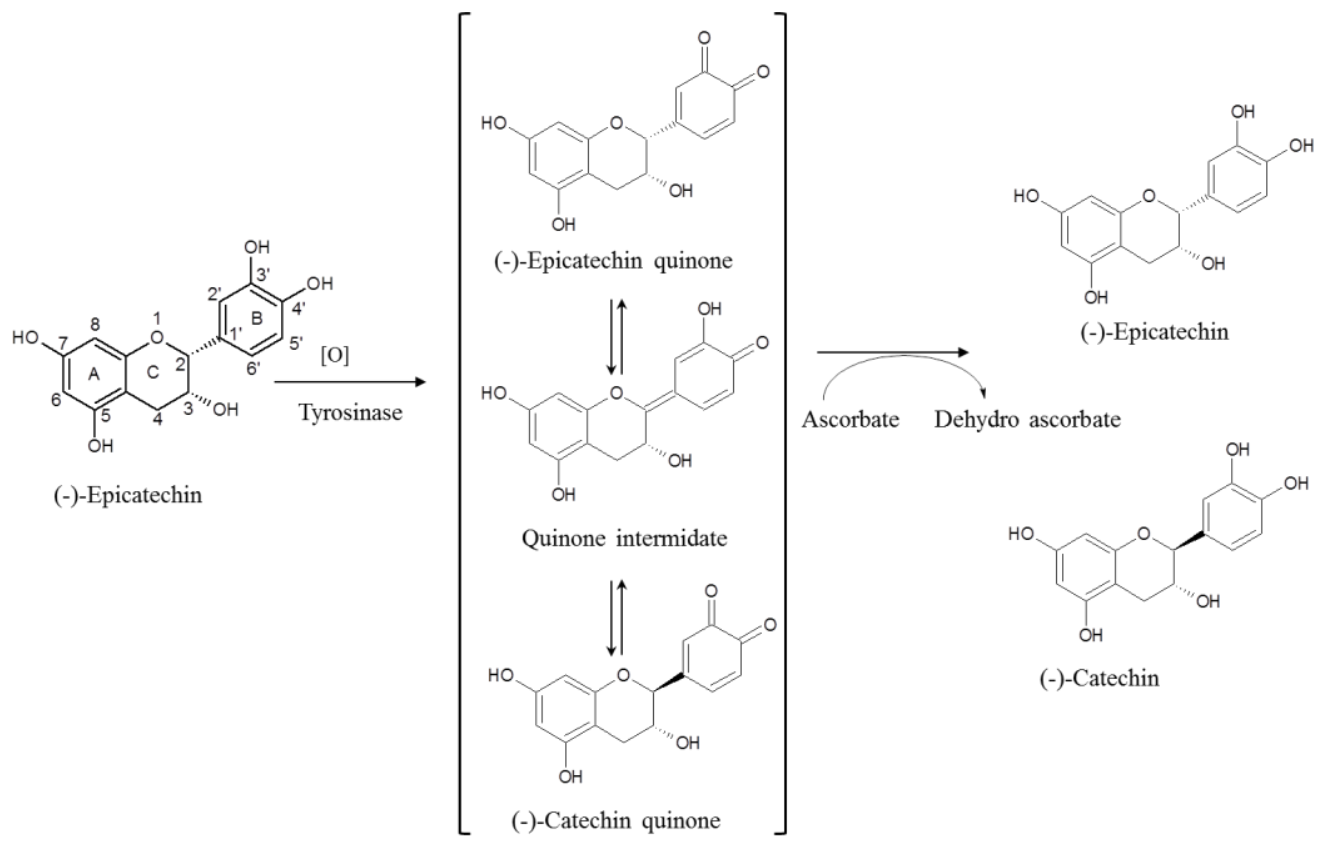

Fig. 1. The investigated epimerization of (-)-epicatechin to (-)-catechin under the interplay of tyrosinase and ascorbate. (-)-Epicatechin is oxidized by tyrosinase to form (-)-epicatechin quinone. Three possible tautomeric forms for the quinone are depicted and include (-)-epicatechin quinone and (-)-catechin quinone. Reduction of (-)epicatechin quinone by ascorbate gives (-)-epicatechin. Reduction of (-)-catechin quinone by ascorbate gives (-)catechin. If the proposed oxidation, tautomerizaion and recycling takes place, this will lead to partial epimerization of (-)-epicatechin to (-)-catechin.

\section{Materials and methods}

\subsection{Chemicals}

(-)-Epicatechin, (-)-catechin, tyrosinase, ascorbate and GSH were purchased from Sigma (St. Louis, MO, USA) and dissolved in assay buffer (100 $\mathrm{mM}$ ammonium bicarbonate, $\mathrm{pH} 7.4)$.

\section{2. (-)-Epicatechin oxidation}

The oxidation of $50 \mu \mathrm{M}$ (-)-epicatechin was performed at $37{ }^{\circ} \mathrm{C}$ in the assay buffer and started by adding $10 \mathrm{U} / \mathrm{mL}$ tyrosinase. After starting the oxidation, the UV absorption spectrum $(\lambda=200-600 \mathrm{~nm}$ ) was recorded every $2 \mathrm{~min}$ for $10 \mathrm{~min}$ (see legend of Fig. 2). In parallel, $50 \mu \mathrm{M}$ ascorbate or $100 \mu \mathrm{M}$ GSH were added to (-)-epicatechin before starting the reaction $[14,20]$. The ascorbate consumption was determined from the absorption at $270 \mathrm{~nm}$ using a molar extinction coefficient of $3640 \mathrm{M}^{-1} \cdot \mathrm{cm}^{-1}$. The reactions were first monitored spectrophotometrically for $5 \mathrm{~min}$ and then analyzed with HPLC and UHPLC-Q-TOF/MS. 


\subsection{HPLC analysis}

High performance liquid chromatography (HPLC) coupled to ultraviolet detection at $230 \mathrm{~nm}$ was performed using an Agilent $1260 \mathrm{HPLC}$ system and a diode array detector (Agilent Technologies, Santa Clara (CA), USA). An Apollo C18 column $(150 \times 4.60 \mathrm{~mm}$ i.d., $5 \mu \mathrm{m})$ was used. The mobile phase was composed of solvent $\mathrm{A}\left(\mathrm{H}_{2} \mathrm{O}\right)$ and solvent $\mathrm{B}$ (acetonitrile). The gradient program was: 0-2 min: 96-92\% A, 2-11 min: 92-75\% A. After each injection, $90 \%$ of $\mathrm{B}$ was used to wash the column for $5 \mathrm{~min}$, and the column was reequilibrated with $4 \%$ of B for $5 \mathrm{~min}$. The flow rate was $1.0 \mathrm{~mL} / \mathrm{min}$ and $20 \mu \mathrm{L}$ of samples were injected $[21,22]$.

\subsection{UHPLC-ESI-Q-TOF/MS and MS/MS analysis}

The reaction products were separated using an Agilent 1290 infinity ultrahigh performance liquid chromatography (UHPLC) system (Agilent Technologies, Santa Clara (CA), USA). The UHPLC was equipped with a quaternary pump with an integrated vacuum degasser (G4204A), an autosampler (G4226A) with a thermostat (G1330B), and a thermostatted column compartment (G1316C). An Agilent Extend C18 column $(2.1 \times 50$ $\mathrm{mm}, 1.8 \mu \mathrm{m})$, with an Agilent Eclipse plus C18 guard column $(2.1 \times 5 \mathrm{~mm}, 1.8 \mu \mathrm{m})$, was selected as the stationary phase. The mobile phase consists of a mixture of $0.1 \%(\mathrm{v} / \mathrm{v})$ formic acid in water (solvent A) and acetonitrile (solvent B).

The flow rate was set to $0.4 \mathrm{~mL} / \mathrm{min}$, and the column temperature was maintained at $25^{\circ} \mathrm{C}$. The gradient elution started with $10 \% \mathrm{~B}$, increased linearly to $35 \% \mathrm{~B}$ over $4 \mathrm{~min}$, and continued to increase to $75 \%$ solvent B over 4.75 min before decreasing to $10 \% \mathrm{~B}$ over $5.50 \mathrm{~min}$. An equilibrium period of $1.25 \mathrm{~min}$ was used between two injections. The injection volume was $10 \mu \mathrm{L}$.

(-)-Epicatechin metabolites were detected using an Agilent 6550 iFunnel Accurate-Mass Quadrupole Time-of-Flight Mass Spectrometer (Q-TOF/MS) equipped with Dual Agilent Jet Stream technology for electrospray ionization (ESI) interface in the negative ionization mode. The capillary voltage was set to $2500 \mathrm{~V}$. The temperature and flow rate of the drying gas were set to $150^{\circ} \mathrm{C}$ and $20 \mathrm{~L} / \mathrm{min}$, respectively, while the temperature and flow rate of the sheath gas were $350^{\circ} \mathrm{C}$ and $12 \mathrm{~L} / \mathrm{min}$, respectively. The nebulizer pressure was controlled to $25 \mathrm{psi}$. The mass range for the TOF scanning was set to $50-1700 \mathrm{~m} / \mathrm{z}$.

Peaks of eluents corresponding to (-)-epicatechin and (-)-epicatechin metabolites were subjected to Q-TOF MS/MS analysis to achieve a high mass accuracy. The collision energies were fixed to 35 and 32 for (-)-catechin and (-)-epicatechin, respectively. The $\mathrm{m} / \mathrm{z}$ range for the targeted MS/MS acquisition was set to $50-1700 \mathrm{~m} / \mathrm{z}$ in negative ESI mode. The mass accuracy was constantly checked via online calibration using a calibration solution containing the internal reference ions at $\mathrm{m} / \mathrm{z}$ at 112.9855 and 1033.9881 in negative ionization mode. Data were analyzed and processed using Agilent Mass Hunter Workstation Quantitative and Qualitative Analysis software B.06.01. Structure confirmation of epicatechin metabolites was obtained by Agilent Mass Hunter structure correlator software $[23,24]$. 


\subsection{LUMO map of (-)-epicatechin quinone.}

Quantum molecular calculations were performed using Spartan'16 to analyze the tautomers of the oxidized (-)-epicatechin. The Lowest Unoccupied Molecular Orbital (LUMO) energies $(\mathrm{kJ} / \mathrm{mol})$ of the tautomers of (-)-epicatechin quinone were calculated as well as their equilibrium geometry under Hartree-Fock (HF) 6-31G* basis set. The dihedral angles between the plane of the AC-ring and that of the B-ring of (-)-epicatechin quinones were also determined [17,25].

\subsection{Statistics}

All experiments were performed at least in triplicate. Results are shown as mean \pm standard error of the mean (S.E.M.) or as a typical example.

\section{Results}

A

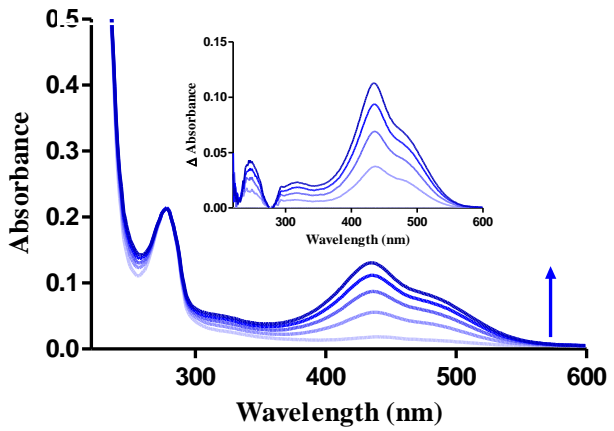

Wavelength (nm)

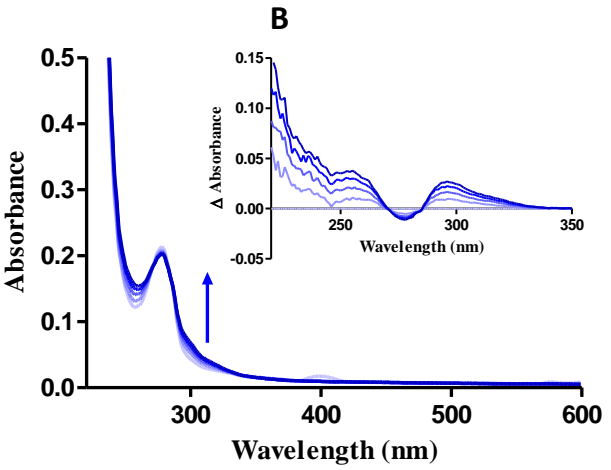

Wavelength $(\mathbf{n m})$

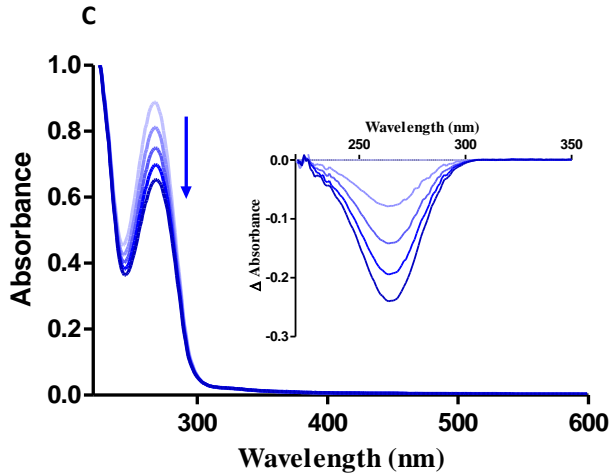

Fig. 2. The UV spectra of the incubation mixture containing $50 \mu \mathrm{M}(-)$-epicatechin and $10 \mathrm{U} / \mathrm{mL}$ tyrosinase (A). The same experiment was carried out in the presence of $100 \mu \mathrm{M}$ GSH (B) or $50 \mu \mathrm{M}$ ascorbate $(\mathbf{C})$. The reaction was started by the addition of tyrosinase. UV scans were recorded every 2 min after starting the reaction. The insert shows the difference in absorption of each scan compared with that of the first scan. A typical example is shown. 

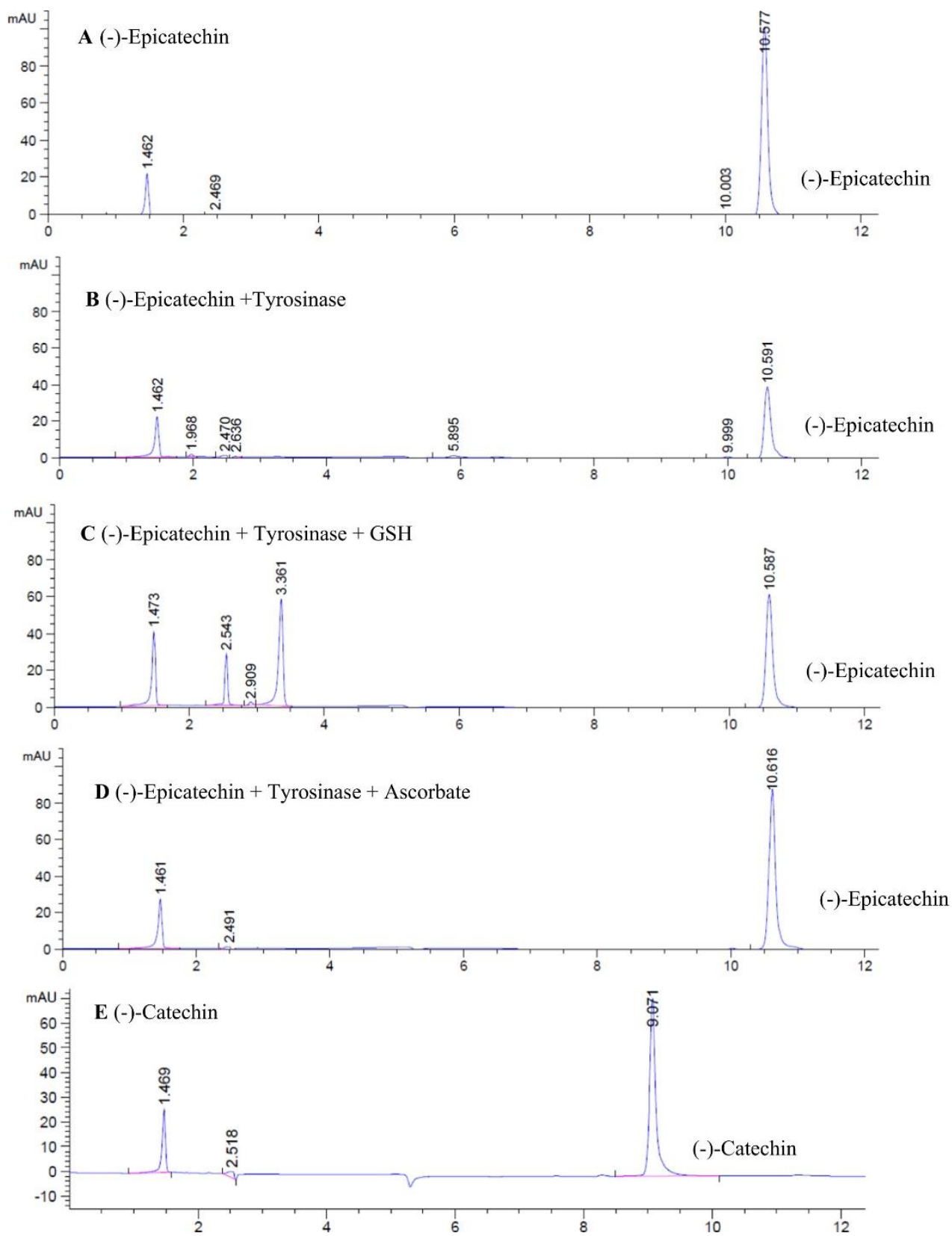

Fig. 3. HPLC chromatograms of $50 \mu \mathrm{M}$ (-)-epicatechin (A) and $50 \mu \mathrm{M}(-)$-catechin (E) calibration standard, and of the incubation mixture containing $50 \mu \mathrm{M}(-)$-epicatechin and $10 \mathrm{U} / \mathrm{mL}$ tyrosinase (B), in presence of $50 \mu \mathrm{M}$ ascorbate (C) or $100 \mu \mathrm{M}$ GSH (D). The incubation mixtures were injected into the HPLC system 5 min after starting the reaction with tyrosinase. The retention time of (-)-epicatechin and (-)-catechin are 10.6 and 9.1, respectively. The consumption of (-)-epicatechin in $\mathrm{B}, \mathrm{C}$ and $\mathrm{D}$ is $16.71 \pm 1.34 \mu \mathrm{M}, 17.041 \pm 2.24 \mu \mathrm{M}$ and $3.04 \pm 0.76$ $\mu \mathrm{M}$, respectively. 

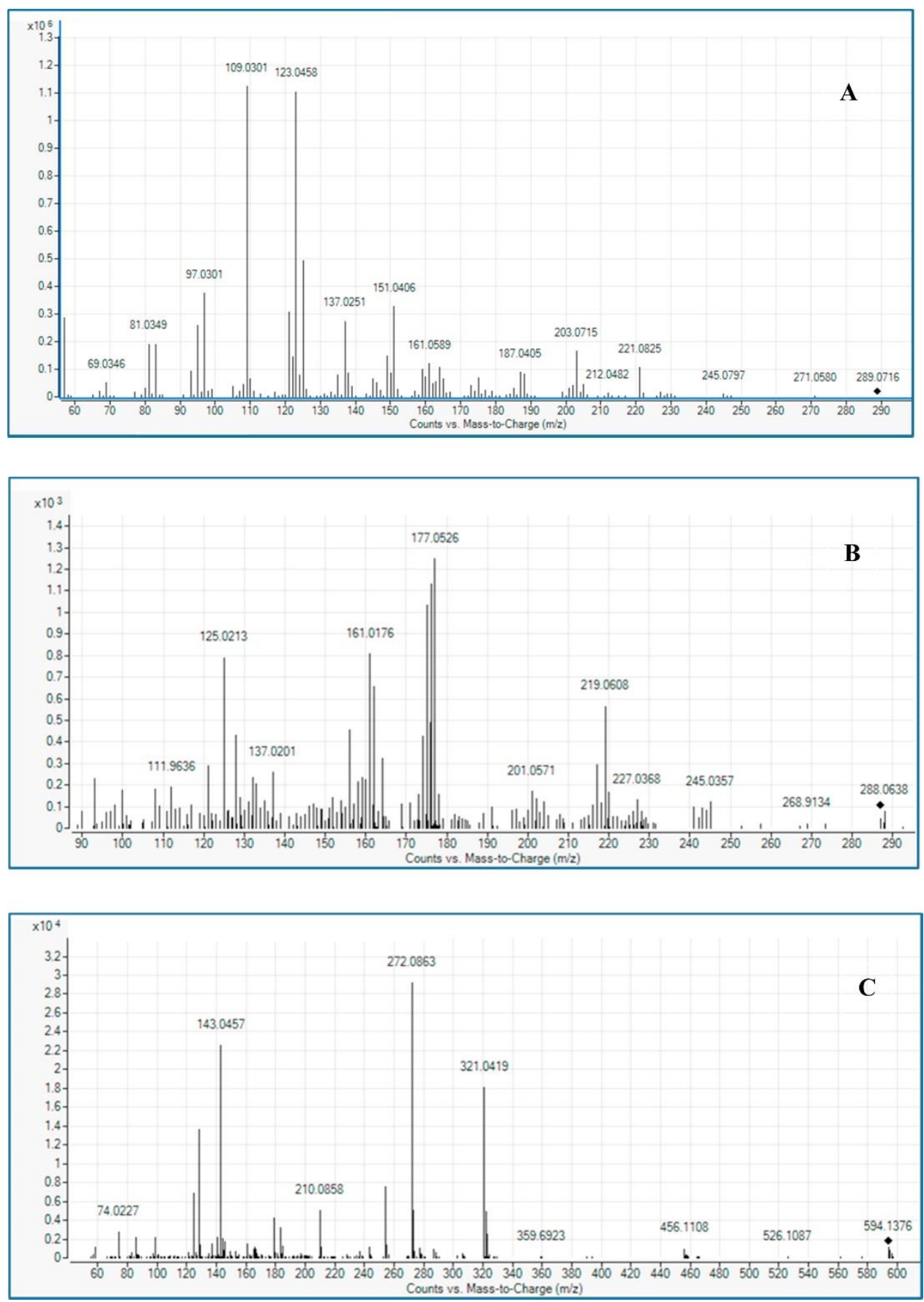

Fig. 4. Q-TOF MS/MS spectra of (-)-epicatechin (A), (-)-epicatechin quinone (B) and (-)-epicatechin-GSH adducts (C) with characteristic fragment ions. 


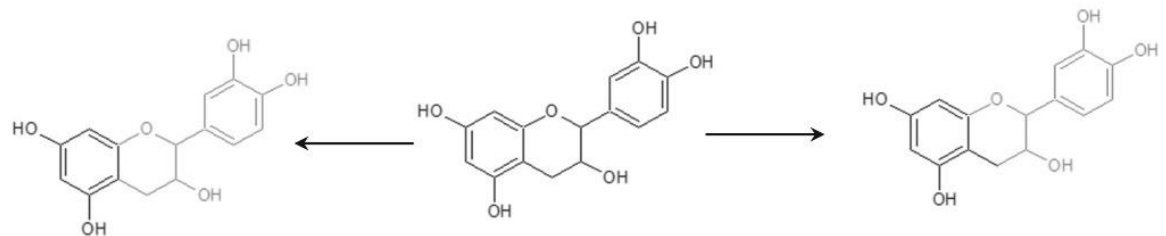

Fragment m/z 109.0301

$\mathrm{m} / \mathrm{z} 289.0716$

Fragment m/z 123.0458

(-)-Epicatechin<smiles>O=C1C=CC(C2Oc3cc(O)cc(O)c3CC2O)=CC1=O</smiles>

Fragment m/z 161.0176<smiles>O=C1C=CC(C2Oc3cc(O)cc(O)c3CC2O)=CC1=O</smiles>

Fragment m/z 177.0526

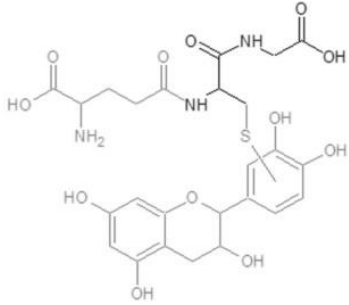

Fragment m/z 143.0457

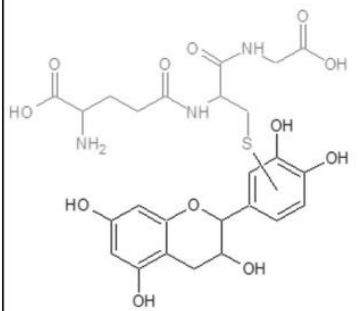<smiles>NC(CCC(=O)NC(CSCCO)C(=O)NCC(=O)O)C(=O)O</smiles>

$\mathrm{m} / \mathrm{z} 288.0638$
(-)-Epicatechin quinone

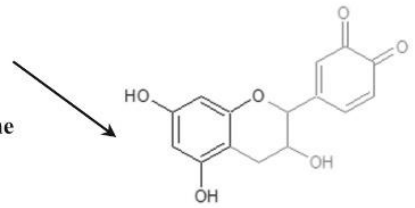

Fragment m/z 125.0213

Fragment m/z 321.0419

Fig. 5. Fragmentation patterns for (-)-epicatechin, (-)-epicatechin quinone and (-)-epicatechin-GSH adducts obtained from the MS/MS spectra using MassHunter MSC software. The chemical structures of the fragments are highlighted in black within the chemical structure of the parent molecules. 


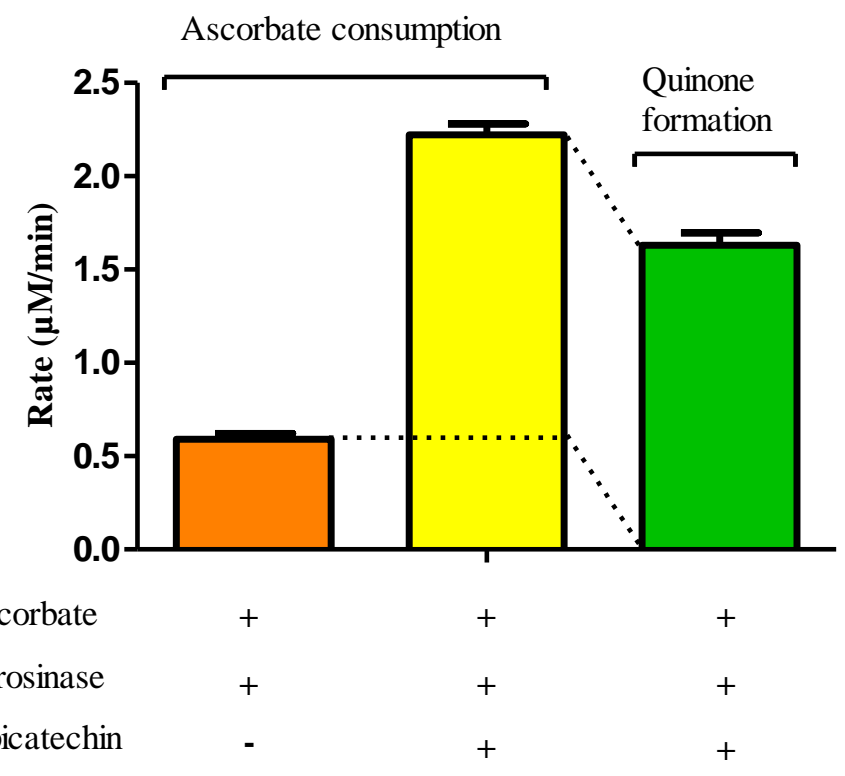

Fig. 6. The rate of ascorbate consumption and (-)-epicatechin quinone formation in the incubation mixture containing $50 \mu \mathrm{M}$ ascorbate, $10 \mathrm{U} / \mathrm{mL}$ tyrosinase or $50 \mu \mathrm{M}$ (-)-epicatechin. The reaction was started with the addition of tyrosinase. Ascorbate consumption was calculated from the reduction in the absorption at $270 \mathrm{~nm}$ using a molar extinction coefficient of $3640 \mathrm{M}^{-1} \cdot \mathrm{cm}^{-1}$. The difference between the ascorbate consumption with and without (-)-epicatechin was ascribed to reduction of the (-)-epicatechin quinone generated by the tyrosinase catalyzed oxidation of (-)-epicatechin. This was used to estimate the rate of (-)-epicatechin quinone formation. 


\begin{tabular}{|c|c|c|c|c|}
\hline & Quinone intermediate & (-)-Catechin quinone \\
\hline structure
\end{tabular}

Fig. 7. The tautomers of (-)-epicatechin quinone, their Lowest Unoccupied Molecular Orbital (LUMO) map, and dihedral angle between the plane of the AC-ring and that of the B-ring. In depicting the dihedral angle, only $\mathrm{C}_{2}$ and $\mathrm{C}_{3}$ of the AC-ring are shown for sake of clarity. The large difference in the dihedral angle is indicative for the high energy barrier between the tautomers and can explain why the proposed tautomerization does not take place. 


\section{1. (-)-Epicatechin is oxidized by tyrosinse into (-)-epicatechin quinone}

Addition of tyrosinase to (-)-epicatechin resulted in the oxidation of (-)-epicatechin, which was evident from the change in the UV spectrum (Fig. 2A). HPLC analysis of the incubation mixture confirmed that (-)-epicatechin was consumed during the oxidation process (Fig. 3B). The Q-TOF MS/MS analysis showed that (-)-epicatechin quinone was formed (Fig. 4B). (-)-Epicatechin quinone yielded fragment ions at $288.06 \mathrm{~m} / \mathrm{z}, 177.05 \mathrm{~m} / \mathrm{z}$, $161.02 \mathrm{~m} / \mathrm{z}$ and $125.02 \mathrm{~m} / \mathrm{z}$. The protonated and fragmented ions of (-)-epicatechin were $289.07 \mathrm{~m} / \mathrm{z}, 109.03 \mathrm{~m} / \mathrm{z}$ and $123.05 \mathrm{~m} / \mathrm{z}$ (Fig. 4A). The diastereoisomers, (-)-epicatechin and (-)-catechin, gave almost the same fragment ions indicating that the isomers could not be distinguished by only using their mass spectra. Using HPLC analysis, (-)-epicatechin and (-)-catechin showed a relatively large difference in the retention time, which were 10.6 min and $9.1 \mathrm{~min}$ respectively (Fig. 3). This was used to selectively detect both diastereoisomers.

\subsection{The confirmation of the formation of (-)-epicatechin quinone}

When (-)-epicatechin is oxidized by tyrosinase in the presence of GSH, the change in the UV spectrum indicated that (-)-epicatechin-GSH adducts were formed (Fig. 2B). HPLC analysis shows new peaks in the chromatogram at 2.9 and $3.4 \mathrm{~min}$ (Fig. 3C) also indicating the formation of GSH adducts. Q-TOF/MS analysis of (-)-epicatechin interacting with tyrosinase and GSH confirms the presence of (-)-epicatechin-GSH adducts, based on the characteristic fragment ions of (-)-epicatechin-GSH of $594.14 \mathrm{~m} / \mathrm{z}, 272.09 \mathrm{~m} / \mathrm{z}, 143.05 \mathrm{~m} / \mathrm{z}$ and $321.04 \mathrm{~m} / \mathrm{z}$ (Fig. 4C). Matching the observed MS/MS fragments with the proposed structure provides additional evidence for the formation of (-)-epicatechin-GSH adducts (Fig. 5). The formation of (-)-epicatechin-GSH adducts confirms that tyrosinase converts ()-epicatechin into its quinone. It is concluded that (-)-epicatechin is first converted into (-)epicatechin quinone, and subsequently the quinone reacts with GSH into (-)-epicatechinGSH adducts.

\subsection{No epimerization of (-)-epicatechin quinone to (-)-catechin quinone}

When (-)-epicatechin is oxidized by tyrosinase in the presence of ascorbate, the UV recordings indicate that there is no net consumption of (-)-epicatechin. However, the spectral change observed at $270 \mathrm{~nm}$ indicates that ascorbate is greatly consumed in the presence of (-)-epicatechin and tyrosinase (Fig. 2C). The rate of ascorbate consumption with (-)-epicatechin and tyrosinase $(2.22 \pm 0.06 \mu \mathrm{M} / \mathrm{min})$ is much higher than that of ascorbate with tyrosinase $(0.60 \pm 0.03 \mu \mathrm{M} / \mathrm{min})$ (Fig. 6). HPLC analysis confirmed that hardly any net oxidation of (-)-epicatechin by tyrosinase occurs in the presence of ascorbate (Fig. 3D). These results indicate that in the incubation with ascorbate, (-)-epicatechin is first oxidized into (-)-epicatechin quinone and that the quinone immediately reacts with ascorbate to regenerate (-)-epicatechin. Assuming that ascorbate and (-)-epicatechin quinone react $1: 1$, the rate of (-)-epicatechin quinone formation equals to ascorbate consumption by tyrosinase, which is $1.63 \pm 0.07 \mu \mathrm{M} / \mathrm{min}$ (Fig. 5).

When (-)-epicatechin is oxidized by tyrosinase in the presence of ascorbate, (-)epicatechin quinone is formed. This quinone might be stabilized by epimerization, with the tautomeric forms given in Fig. 1 . In the quinone intermediate, the $C_{2}-C_{1}$ ' bound changes from a single bound to a double bound. Converting this double bound back to a single 
bound can be achieved in two manners and therefore this would lead to (partial) epimerization at the asymmetric $\mathrm{C}_{2}$ carbon. This would result in (partial) conversion of (-)epicatechin quinone into (-)-catechin quinone. Due to the limited stability of the quinone, this was not examined directly by examining the epimerization of the quinone itself.

In the presence of ascorbate, the quinone is not degraded but immediately reduced. If the epimerization of the quinone would take place, the reduction of the quinone by ascorbate would yield (-)-catechin as well as (-)-epicatechin. As described above, the epimerization of (-)-epicatechin to (-)-catechin can be examined using HPLC since they are diastereoisomers.

In the incubation of (-)-epicatechin with tyrosinase and ascorbate, no appreciable amount of (-)-catechin was detected using HPLC. This indicates that there is no substantial epimerization of (-)-epicatechin and (-)-catechin during oxidation. In supplementary information, the LC/MS chromatograms obtained in conformation of the identity of the (-)epicatechin quinone and (-)-epicatechin-GSH adducts, are shown. A trace of (-)-catechin was detected in the incubation of (-)-epicatechin with tyrosinase and ascorbate. A similar trace of (-)-catechin was detected in the (-)-epicatechin calibration standard and in the incubation of (-)-epicatechin without tyrosinase and ascorbate. That only traces of (-)catechin were detected confirms the HPLC result that no substantial epimerization takes place.

\subsection{The LUMO energy of (-)-epicatechin quinone}

In the oxidative activity of (-)-epicatechin quinone, the LUMO accepts the electrons and is thus pivotal in its reactivity. In Fig. 7, it is shown how the energy of the LUMO orbital is distributed over the molecule in the three tautomers of (-)-epicatechin quinone. With the energy of $136.04 \mathrm{~kJ} / \mathrm{mol}$, the LUMO of quinone intermediate is located in B-ring and for a small part also located in the C-ring. The LUMO of (-)-epicatechin quinone and (-)catechin quinone are concentrated in B-ring, and their energies are $44.38 \mathrm{~kJ} / \mathrm{mol}$ and 36.66 $\mathrm{kJ} / \mathrm{mol}$ respectively. The high energy difference between (-)-epicatechin quinone (44.38 $\mathrm{kJ} / \mathrm{mol})$ and the quinone intermediate $(136.04 \mathrm{~kJ} / \mathrm{mol})$ indicates that the formation of quinone intermediate is not likely to occur. The calculations also show that there is a relatively large difference in the dihedral angle between the three tautomers of (-)epicatechin quinone. The large difference in dihedral angle between (-)-epicatechin quinone and the quinone intermediate also indicates that there is no substantial isomerization. This further corroborates that the chemical reactivity of (-)-epicatechin quinone mainly resides in its B-ring.

\section{Discussion}

Many studies have proved that dietary antioxidants from food support the cellular defense system in dealing with oxidative stress [26]. (-)-Epicatechin, one of the most important dietary antioxidants, has gained significant attention because of its potent antioxidant activity [27]. It has been proved that the B-ring of (-)-epicatechin, a catechol moiety, is essential for its direct antioxidant activity to scavenge ROS [28]. When scavenging ROS, (-)-epicatechin will be oxidized into its two-electron oxidation product, ()-epicatechin quinone. This quinone is much less reactive than the ROS scavenged, which is pivotal in the redox modulating effect of (-)-epicatechin [29]. Nevertheless, this quinone has taken over part of the reactivity of the ROS scavenged, and it can react e.g. with thiol 
groups. The research aim of the study is to unravel one aspect of (-)-epicatechin quinone, namely its stabilization. Looking at its chemical structure, (-)-epicatechin quinone might be stabilized by the tautomerization as depicted in Fig. 1.

In present study, (-)-epicatechin quinone was formed using tyrosinase. GSH is known to adduct quinones. The formation of (-)-epicatechin quinone was supported by the (-)epicatechin-GSH adducts found when GSH was added to the incubation of (-)-epicatechin with tyrosinase. The formed (-)-epicatechin-GSH adducts were detected using UV, HPLC and Q-TOF /MS. Moreover, the formation of (-)-epicatechin quinone was also confirmed with Q-TOF /MS detection. To our knowledge this is the first time that (-)-epicatechin quinone was directly detected.

Quinones of polyphenols have a short half-life. Therefore, ascorbate was used to reduce the quinones formed during the tyrosinase catalyzed oxidation of (-)-epicatechin to the corresponding more stable polyphenols. If the tautomerization of the (-)-epicatechin quinone to the (-)-catechin quinone occurs (as depicted in Fig. 1), the reduction by ascorbate will result not only in the recycling of (-)-epicatechin, but also in the formation of (-)-catechin.

That ascorbate indeed directly reduces the (-)-epicatechin quinone formed into (-)epicatechin is shown by the UV and HPLC results of the incubation mixture containing (-)epicatechin, tyrosinase and ascorbate. In this incubation system, ascorbate is consumed, while (-)-epicatechin is instantaneously recycled and therefore the concentration of (-)epicatechin stays the same. The HPLC coupled with UV detection does not show the formation of a detectible amount of (-)-catechin. This indicates that no appreciable epimerization of (-)-epicatechin quinone to (-)-catechin quinone occurs.

The LC/MS chromatograms presented in supplementary information shows that a trace of (-)-catechin was detected in the incubation mixture of (-)-epicatechin with tyrosianse and ascorbate. Similarly, a trace of (-)-catechin was also detected in the (-)-epicatechin calibration standard and in the incubation of (-)-epicatechin without tyrosinase and ascorbate. The later findings indicate that the trace amount of (-)-catechin found are formed by "spontaneous epimerization" of (-)-epicatechin.

In the epimerization of (-)-epicatechin quinone to (-)-catechin quinone, the relatively big difference in the dihedral angle (the angle between the plane of AC-ring and that of the Bring) between (-)-epicatechin quinone and the quinone intermediate will cause an energy barrier that is hard to overcome. In the spontaneous epimerization of (-)-epicatechin (dihedral angle $-57.03^{\circ}$ ) to (-)-catechin (dihedral angle $-55.74^{\circ}$ ), the overall change in dihedral angle is similar to that of the corresponding quinones (from $-55.96^{\circ}$ to $55.55^{\circ}$, Fig. 7). A difference is that the C-ring is opened in the intermediate of the spontaneous isomerization (Fig. 8) and there is no fixed "dihedral angle" between the ring systems because the C-ring is not a ring anymore [30]. This makes the quinone intermediate of spontaneous isomerization much more flexible than the rigid quinone intermediate depicted in Fig. 1. Therefore, the conformational change from (-)-epicatechin to the flexible intermediate is relatively small compared to the big conformational change from (-)epicatechin quinone to the rigid quinone intermediate. This suggests that the spontaneous epimerization of (-)-epicatechin is quicker than the epimerization of (-)-epicatechin quinone. Actually, that only traces of (-)-catechin were detected confirms the HPLC data that no substantial epimerization takes place. 
<smiles>Oc1cc(O)c2c(c1)O[C@H](c1ccc(O)c(O)c1)[C@H](O)C2</smiles>

(-)-Epicatechin

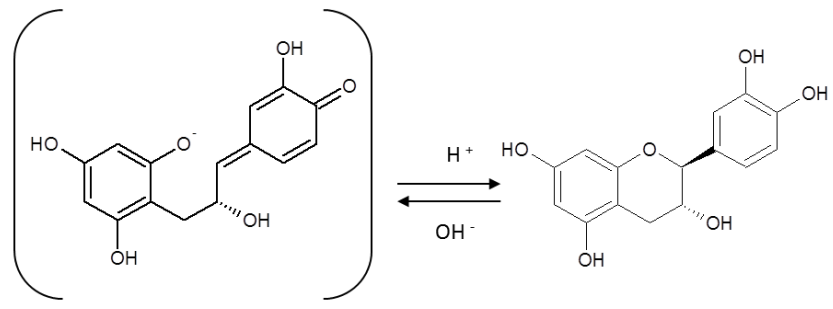

(-)-Catechin

Fig. 8. Proposed mechanism for the spontaneous epimerization of (-)-epicatechin to (-)-catechin [30].

The reactivity of (-)-epicatechin quinone, that is primarily due to its ability to easily accept electrons, was also evaluated by constructing its LUMO map. It shows that the LUMO energy of (-)-epicatechin quinone is indeed concentrated in the B-ring. Within the B-ring, the most electron deficient positions of (-)-epicatechin quinone are on the 2', 5' and 6 ' position. Consequently, nucleophiles such as GSH are expected to adduct to the B-ring at these positions. Previously, it has been reported that indeed these mono GSH adducts in Bring are formed [31]. This further confirms that the chemical reactivity of (-)-epicatechin quinone primarily resides in B-ring.

When quercetin, another important polyphenol, is oxidized, it will also generate a quinone that can react with thiols. In contrast to (-)-epicatechin quinone, GSH will adduct to the 6 and 8 position of the A-ring of quercetin quinone. This indicates that the chemical reactivity of quercetin quinone does not reside only in B-ring but is spread out over the ACring as well as the B-ring, so over the entire molecule [14,32]. With a high concentrated electron density in B-ring, (-)-epicatechin quinone is harder than quercetin quinone that has its electron density spread over the molecule. According to Pearson's Hard and Soft Acids and Bases concept, "hard" electrophiles will more likely react with "hard" nucleophiles and "soft" electrophiles will more likely react with "soft" nucleophiles [33]. It is obvious that such a difference in chemical reactivity between these bioactives will be reflected in a difference in their biological effects. However, this is still mainly a "terra incognita". The present study is a step further in the differentiation between bioactive polyphenols to finally come up with a rational selection for the appropriate compound for a specific disorder. We would like to stress that the difference between "hard" and "soft" redox modulating compounds should be acknowledged and further elaborated in clinical studies with polyphenols.

\section{Conclusions}

(-)-Epicatechin oxidation involves two electron oxidation of its B-ring, giving (-)epicatechin quinone. Our results show that there is no epimerization of (-)-epicatechin quinone and (-)-catechin quinone in the oxidation process. Therefore, it is concluded that the chemical reactivity of (-)-epicatechin quinone mainly resides in its B-ring. 


\section{Supplementary material}
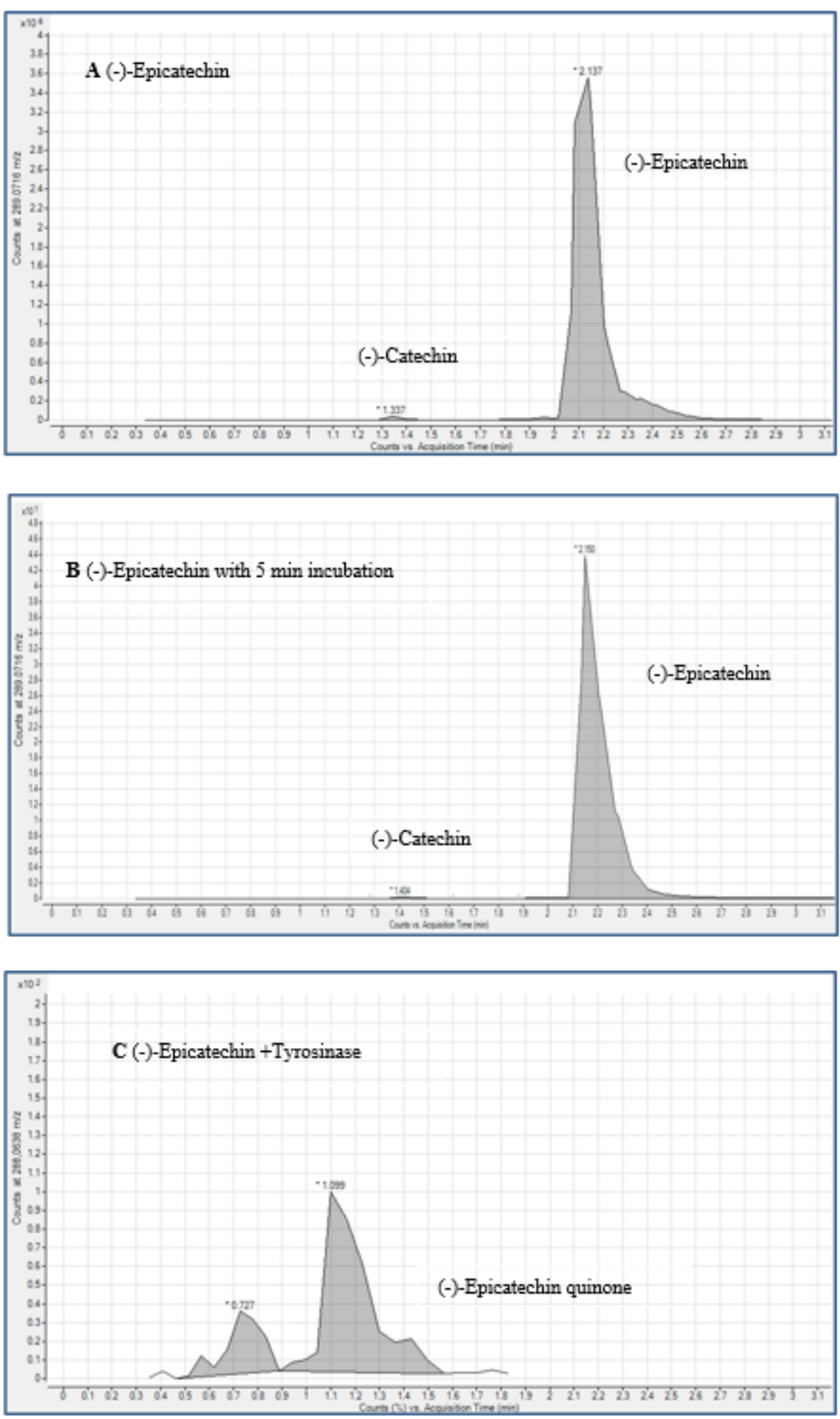

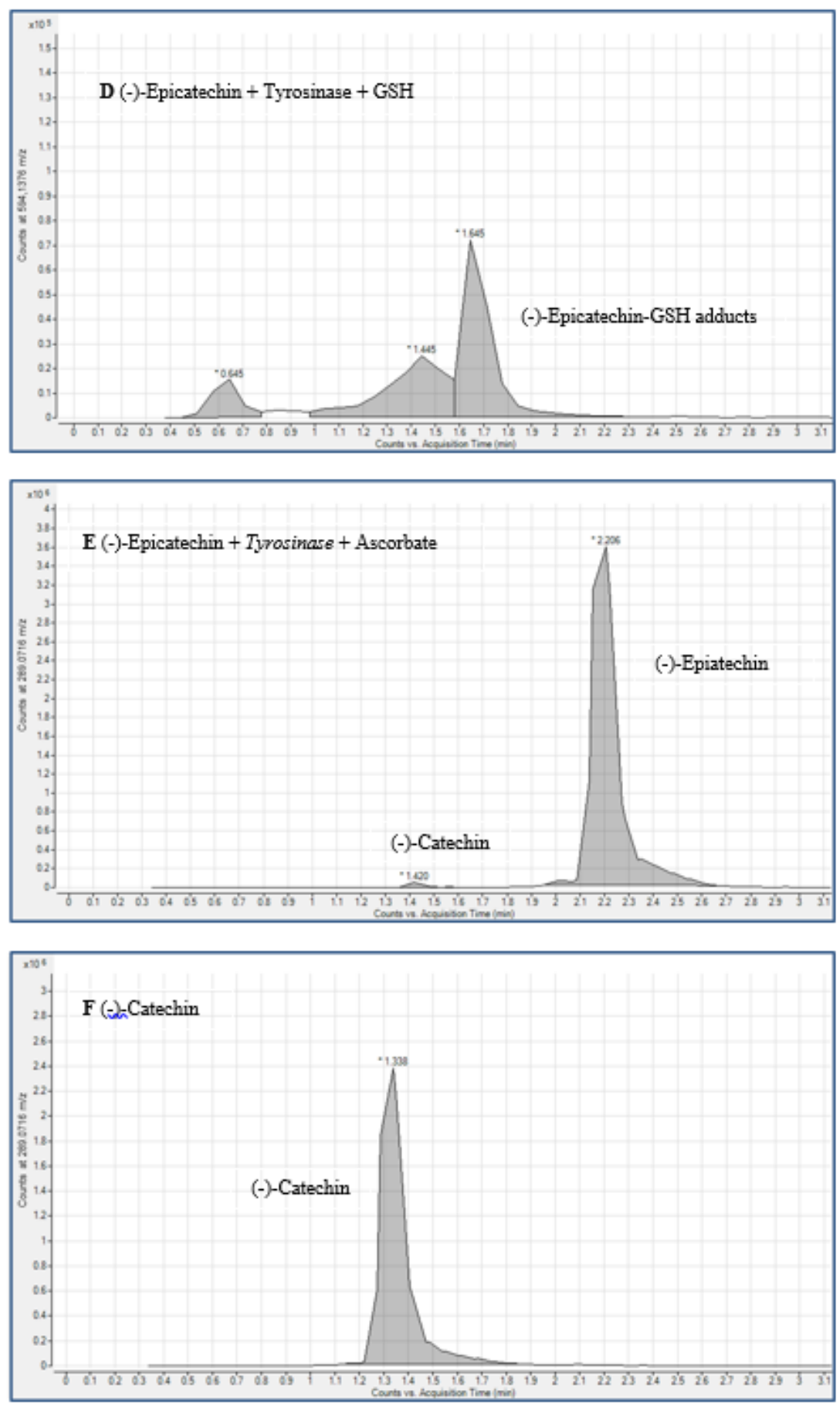


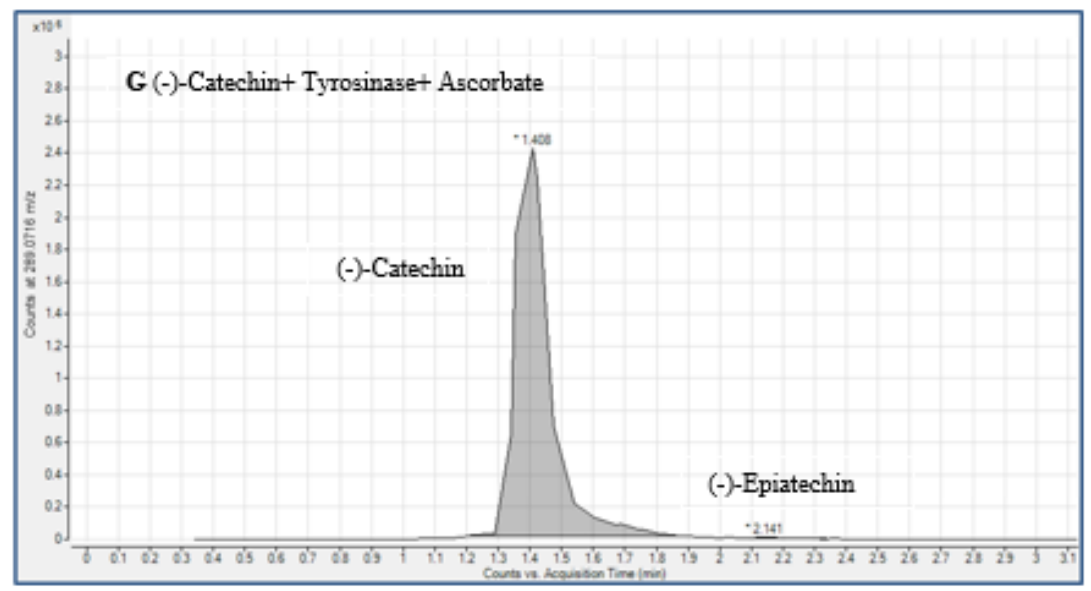

Fig. 1. LC/MS Chromatograms of the $50 \mu \mathrm{M}$ (-)-epicatechin calibration standard (A) and its incubation for 5 min (B), of the incubation mixture containing $50 \mu \mathrm{M}(-)$-epicatechin and $10 \mathrm{U} / \mathrm{mL}$ tyrosinase (C), in presence of 100 $\mu \mathrm{M}$ GSH (D) or $50 \mu \mathrm{M}$ ascorbate (E), of the $50 \mu \mathrm{M}(-)$-catechin calibration standard (F), and of the incubation mixture containing $50 \mu \mathrm{M}(-)$-catechin and of $15 \mathrm{U} / \mathrm{mL}$ tyrosinase and $50 \mu \mathrm{M}$ ascorbate $(\mathrm{G})$. The incubation mixtures were injected into the UHPLC-Q-TOF/MS system $5 \mathrm{~min}$ after starting the reaction with tyrosinase. Traces of (-)-catechin (as evidenced by the shape of the "peak" of (-)-catechin, the concentration is below the Lower Limit of Quantification) were found in the (-)-epicatechin calibration standard (A), as well as in the incubation of (-)-epicatechin without (B) and with tyrosinase and ascorbate (E). Similarly, a trace of (-)epicatechin was found in the incubation of (-)-catechin with tyrosinase in the presence of ascorbate (G). No (-)epicatechin was detected in the (-)-catechin calibration standard (F). The presence of (-)-catechin in chromatogram E might indicate that there is some minor epimerization of (-)-epicatechin quinone. However, we also detected similar traces of (-)-catechin in chromatogram A and B that are probably formed by "spontaneous" epimerization, indicating that the (-)-catechin detected in chromatogram E might also be formed by "spontaneous" epimerization. Moreover, that only tiny traces of (-)-catechin were detected actually confirms the HPLC data that there is no substantial epimerization of the (-)-epicatechin to (-)-catechin. 


\section{References}

1. Kohen, R.; Nyska, A. Invited review: Oxidation of biological systems: oxidative stress phenomena, antioxidants, redox reactions, and methods for their quantification. Toxicologic pathology 2002, 30, 620-650.

2. Diplock, A.; Charuleux, J.L.; Crozier-Willi, G.; Kok, F.; Rice-Evans, C.; Roberfroid, M.; Stahl, W.; VinaRibes, J. Functional food science and defence against reactive oxidative species. British Journal of nutrition 1998, 80, S77-S112.

3. Haenen, G.R.; Veerman, M.; Bast, A. Reduction of $\beta$-adrenoceptor function by oxidative stress in the heart. Free radical biology and medicine 1990, 9, 279-288.

4. Alfadda, A.A.; Sallam, R.M. Reactive oxygen species in health and disease. BioMed research international 2012, 2012.

5. Sundar, I.K.; Yao, H.; Rahman, I. Oxidative stress and chromatin remodeling in chronic obstructive pulmonary disease and smoking-related diseases. Antioxidants \& redox signaling 2013, 18, 1956-1971.

6. Lyakhovich, V.; Vavilin, V.; Zenkov, N.; Menshchikova, E. Active defense under oxidative stress. The antioxidant responsive element. Biochemistry (Moscow) 2006, 71, 962-974.

7. Bast, A.; Kaiserová, H.; Den Hartog, G.; Haenen, G.; Van Der Vijgh, W. Protectors against doxorubicininduced cardiotoxicity: flavonoids. Cell biology and toxicology 2007, 23, 39-47.

8. Boots, A.W.; Wilms, L.C.; Swennen, E.L.; Kleinjans, J.C.; Bast, A.; Haenen, G.R. In vitro and ex vivo antiinflammatory activity of quercetin in healthy volunteers. Nutrition 2008, 24, 703-710.

9. Xie, Y.; Yang, W.; Tang, F.; Chen, X.; Ren, L. Antibacterial activities of flavonoids: structure-activity relationship and mechanism. Current medicinal chemistry 2015, 22, 132-149.

10. Bast, A.; Haenen, G.R. The toxicity of antioxidants and their metabolites. Environmental toxicology and pharmacology 2002, 11, 251-258.

11. Biewenga, G.P.; Haenen, G.R.; Bast, A. The pharmacology of the antioxidant lipoic acid. General Pharmacology: The vascular system 1997, 29, 315-331.

12. Halliwell, B. Antioxidants in human health and disease. Annual review of nutrition 1996, 16, 33-50.

13. Jacobs, H.; Moalin, M.; van Gisbergen, M.W.; Bast, A.; van der Vijgh, W.J.; Haenen, G.R. An essential difference in the reactivity of the glutathione adducts of the structurally closely related flavonoids monoHER and quercetin. Free radical biology and medicine 2011, 51, 2118-2123.

14. Boots, A.W.; Kubben, N.; Haenen, G.R.; Bast, A. Oxidized quercetin reacts with thiols rather than with ascorbate: implication for quercetin supplementation. Biochemical and biophysical research communications 2003, 308, 560-565.

15. Bast, A.; Haenen, G.R.; Doelman, C.J. Oxidants and antioxidants: state of the art. The American journal of medicine 1991, 91, S2-S13.

16. Lemmens, K.J.; van de Wier, B.; Vaes, N.; Ghosh, M.; van Zandvoort, M.A.; van der Vijgh, W.J.; Bast, A.; Haenen, G.R. The flavonoid 7-mono-O-( $\beta$-hydroxyethyl)-rutoside is able to protect endothelial cells by a direct antioxidant effect. Toxicology in vitro 2014, 28, 538-543.

17. Moalin, M.; van Strijdonck, G.P.; Bast, A.; Haenen, G.R. Competition between ascorbate and glutathione for the oxidized form of methylated quercetin metabolites and analogues: tamarixetin, 4' O-methylquercetin, has the lowest thiol reactivity. Journal of agricultural and food chemistry 2012, 60, 9292-9297.

18. Kiatgrajai, P.; Wellons, J.; Gollob, L.; White, J.D. Kinetics of epimerization of (+)-catechin and its rearrangement to catechinic acid. The Journal of organic chemistry 1982, 47, 2910-2912.

19. Ishino, N.; Yanase, E.; Nakatsuka, S.-i. Epimerization of tea catechins under weakly acidic and alkaline conditions. Bioscience, biotechnology, and biochemistry 2010, 74, 875-877.

20. Sthijns, M.M.; Schiffers, P.M.; Janssen, G.M.; Lemmens, K.J.; Ides, B.; Vangrieken, P.; Bouwman, F.G.; Mariman, E.C.; Pader, I.; Arnér, E.S. Rutin protects against $\mathrm{H}_{2} \mathrm{O}_{2}$-triggered impaired relaxation of placental arterioles and induces Nrf2-mediated adaptation in human umbilical vein endothelial cells exposed to oxidative stress. Biochimica et Biophysica Acta (BBA)-General Subjects 2017, 1861, 1177-1189.

21. Zou, H.; Ma, Y.; Xu, Z.; Liao, X.; Chen, A.; Yang, S. Isolation of strawberry anthocyanins using high-speed counter-current chromatography and the copigmentation with catechin or epicatechin by high pressure processing. Food chemistry 2018, 247, 81-88.

22. Cai, R.J.; Yin, X.L.; Liu, J.; Zhao, G.Z. Characterization and identification of in vitro metabolites of (-)epicatechin using ultra-high performance liquid chromatography-mass spectrometry. Tropical Journal of pharmaceutical research 2017, 16, 2985-2990.

23. Chang, C.L.; Wu, R.T. Quantification of (+)-catechin and (-)-epicatechin in coconut water by LC-MS. Food chemistry 2011, 126, 710-717.

24. Galaverna, R.S.; Sampaio, P.T.; Barata, L.E.; Eberlin, M.; Fidelis, C. Differentiation of two morphologically similar Amazonian Aniba species by mass spectrometry leaf fingerprinting. Analytical methods 2015, 7, 1984-1990. 
25. Moalin, M.; Van Strijdonck, G.P.; Beckers, M.; Hagemen, G.J.; Borm, P.J.; Bast, A.; Haenen, G.R. A planar conformation and the hydroxyl groups in the $\mathrm{B}$ and $\mathrm{C}$ rings play a pivotal role in the antioxidant capacity of quercetin and quercetin derivatives. Molecules 2011, 16, 9636-9650.

26. Heim, K.E.; Tagliaferro, A.R.; Bobilya, D.J. Flavonoid antioxidants: chemistry, metabolism and structureactivity relationships. The Journal of nutritional biochemistry 2002, 13, 572-584.

27. Shay, J.; Elbaz, H.A.; Lee, I.; Zielske, S.P.; Malek, M.H.; Hüttemann, M. Molecular mechanisms and therapeutic effects of (-)-epicatechin and other polyphenols in cancer, inflammation, diabetes, and neurodegeneration. Oxidative medicine and cellular longevity 2015, 2015.

28. Ruijters, E.J.; Weseler, A.R.; Kicken, C.; Haenen, G.R.; Bast, A. The flavanol (-)-epicatechin and its metabolites protect against oxidative stress in primary endothelial cells via a direct antioxidant effect. European journal of pharmacology 2013, 715, 147-153.

29. Boots, A.W.; Haenen, G.R.; den Hartog, G.J.; Bast, A. Oxidative damage shifts from lipid peroxidation to thiol arylation by catechol-containing antioxidants. Biochimica et Biophysica Acta (BBA)-Molecular and Cell Biology of Lipids 2002, 1583, 279-284.

30. Whalley, W.; Mehta, P. The stereochemistry of some catechin derivatives. Journal of Chemistry Society 1963, 5327.

31. Moridani, M.Y.; Scobie, H.; Salehi, P.; O'Brien, P.J. Catechin metabolism: glutathione conjugate formation catalyzed by tyrosinase, peroxidase, and cytochrome p450. Chemical research in toxicology 2001, 14, 841848 .

32. Moalin, M. Quercetin and its methylated metabolites: the chemical basis of activity. Doctoral thesis, Maastricht University, Maastricht, the Netheralnds, 2014.

33. Pearson, R.G. Hard and soft acids and bases. Journal of the American chemical society 1963, 85, 3533-3539. 


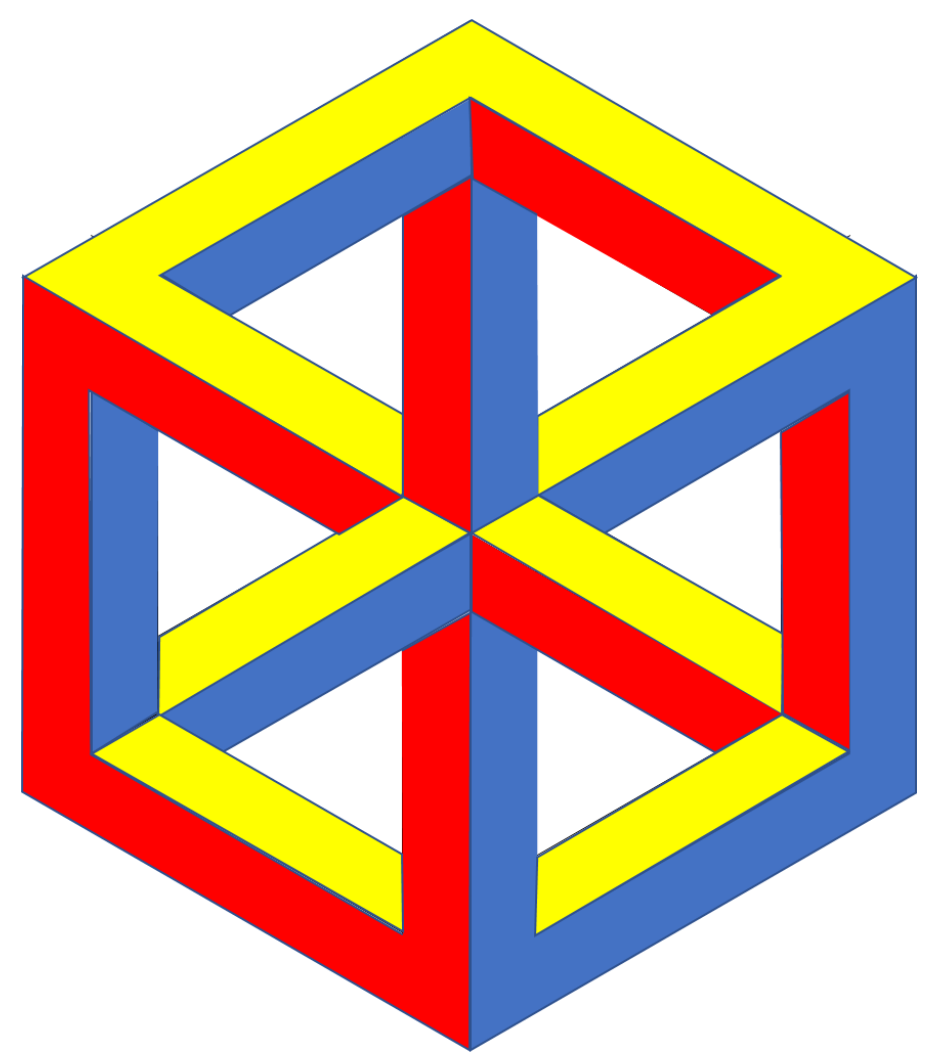





\section{Chapter 5}

\section{Unravelling the redox modulating activity of quercetin}

\section{- a systematic approach -}

Ming Zhang, Mohamed Moalin, Zheng Wen Li, Mohamed Chayoua and Guido R.M.M. Haenen

In preparation. 


\begin{abstract}
Quercetin (Q) was selected to unravel the redox modulating effects of polyphenols, because $\mathrm{Q}$ displays a very strong antioxidant activity. In $\mathrm{Q}$, the $\mathrm{C} 2$ position has the highest occupied molecular orbital because of the interaction of the $3 \mathrm{OH}$ group with the 5 and $7 \mathrm{OH}$ groups, as well as the 4 carbonyl group. Therefore, $\mathrm{Q}$ will donate an electron to a free radical from the $\mathrm{C} 2$ positon. The formed quercetin radical $\left(\mathrm{Q}^{*}\right)$ is stabilized by intra molecular scavenging between $\mathrm{C} 2$ and $\mathrm{C} 1$ ' and the delocalization of the unpaired electron in the B-ring, in which 4'OH group plays an essential role. After intra molecular scavenging, the $\mathrm{AC}$-ring in $\mathrm{Q}^{*}$ is restored to donate another electron to a free radical from the $\mathrm{C} 2$ position. This leads to the formation of the two-electron oxidation product, quercetin quinone methide (QQ). Of the four tautomers of QQ, the tautomer with the carbonyl groups at the maximum distance from each other, is the most abundant one. In line with the hardsoft-acid-base concept, the soft electrophile QQ preferably reacts with soft nucleophiles such as thiols over hard nucleophiles such as ascorbate and water. It is speculated that the adduction of thiol group of KEAP1 by QQ will adapt cells against oxidative stress by increasing the synthesis of endogenous antioxidants. However, direct evidence for the adduction of KEAP1 by QQ has not been proved. Our systematic review paves the path to compare the redox modulating activity of $\mathrm{Q}$ to that of other polyphenols on a molecular level. This forms the fundament to ultimately select the appropriate redox modulating compound to protect against a specific redox mediated disorder.
\end{abstract}

Keywords: redox modulation, quercetin, quercetin radical, quercetin quinone methide, antioxidant activity 


\section{Introduction}

Free radicals are continuously formed in our cells by specific enzymes or as by-products of oxygen metabolism, and these radicals are important in signaling and maintaining homeostasis [1]. Due to the unpaired electron (UE), free radicals are very reactive species that can damage DNA, proteins, lipids and other cellular constituents [2]. To prevent this radical toxicity, our body comprises an intricate antioxidant defense system. However, an excessive production of free radicals will outbalance the defense system and cause oxidative stress [3,4]. The cell damage caused by such a redox imbalance has been implicated in the pathophysiology of various diseases like cancer, diabetes, sarcoidosis, age-related diseases, neurological and neurodegenerative disorders [5,6]. Many studies have proved that dietary antioxidants strengthen the defense system to circumvent damage from oxidative stress, and the intake of these redox modulators is associated with a lower prevalence of oxidative stress induced diseases [7-9].

Polyphenols are common dietary antioxidant compounds present in fruits, vegetables, and other plant-derived foodstuffs [10]. For several decades, they are in the spotlight because of their potent antioxidant activity, which is linked to their anti-proliferative, antidiabetic, anticancer, anti-microbial, anti-inflammatory and antiviral activity $[11,12]$. Estimates on the number of polyphenols present in nature are not consistent, but there are at least several thousand [13]. In addressing their bioactivity, all polyphenols are generally put under the same umbrella. The common denominator is that polyphenols contain several hydroxyl $(\mathrm{OH})$ groups attached to an aromatic ring system. The electron donating effect of these $\mathrm{OH}$ groups boosts the ability of the conjugated ring system to react with free radicals $[14,15]$. It is known that a relatively small change in the molecular structure of a polyphenol can have a drastic effect on its antioxidant activity [16]. Moreover, in addressing the antioxidant effect of a polyphenol, often only one aspect is examined [17]. This limited view is one of the reasons why the biological activity of polyphenols remained enigmatic.

In order to protect against oxidative stress, the first critical step is that the polyphenol has to react as quickly as possible with the free radical to prevent that the free radical can attack cellular constituents like DNA. Thus the rate at which the polyphenol reacts with the free radical is one of the main characteristics determining its redox modulating activity $[18,19]$. In this direct scavenging reaction, an electron is transferred from the polyphenol to the free radical, turning the free radical into a far less reactive non-radical compound. This neutralizing of the reactivity of the free radical accounts for the direct protective effect of the polyphenol [20]. The polyphenol, however, loses an electron and is therefore converted into the polyphenolic radical. Because the UE can be delocalized over the conjugated ring system, the reactivity of the polyphenolic radical is much lower than that of the scavenged free radical [21]. It is often not realized that besides the rate at which the polyphenol scavenges the free radical, the stability of the polyphenolic radical also determines its redox modulating activity. Subsequently, this polyphenolic radical can also donate an electron to a free radical. This leads to the accumulation of two-electron oxidation product of the polyphenol after scavenging free radicals [22].

It has been established that antioxidants do not act in isolation. Together with other antioxidants, they form an intricate network [23]. The two-electron oxidation product of the polyphenol also participates in the antioxidant network [16]. It has been reported that some two-electron oxidation products of polyphenols quickly react with ascorbate, which will 
regenerate the polyphenol. The oxidized ascorbate is reduced to ascorbate at the expense of reduced nicotinamide adenine dinucleotide (NADH) that is oxidized to nicotinamide adenine dinucleotide $\left(\mathrm{NAD}^{+}\right)$. Ultimately, the reactivity is safely channeled through the antioxidant network to be finally neutralized by metabolic network, in which $\mathrm{NAD}^{+}$can be converted into NADH [24]. Alternatively, the two-electron oxidation product of a polyphenol can also adduct thiol groups present in e.g. glutathione (GSH) and cellular proteins $[25,26]$. One of these proteins is KEAP1, and the reaction of the two-electron oxidation products of a polyphenol is reported to activate the antioxidant machinery of the cell [27].

In order to better appreciate and understand the impact of these compounds on health, we selected one of the most important polyphenolic compounds, i.e. quercetin (Q), and tried to unravel the three aspects that are essential in its antioxidant activity. Firstly, we had a look at Q itself; what determines the high scavenging potency of Q. Subsequently, we examined the $\mathrm{Q}$ radical ( $\left.\mathrm{Q}^{*}\right)$; how the reactivity of its $\mathrm{UE}$ is retained within the molecule. Finally, we focused on the two-electron oxidation product, i.e. quercetin quinone methide (QQ); how QQ is formed and how QQ fits within the antioxidant network. Sharpening the picture will help us to understand the molecular mode of action of polyphenols and to see differences between polyphenols, so we will be able to select the appropriate redox modulating compound for a specific redox mediated disorder. 


\section{The main steps in the redox modulating activity of quercetin (Q)}

\section{Step 1: $Q$ donates an electron to a free radical at the $\mathrm{C} 2$ positon}

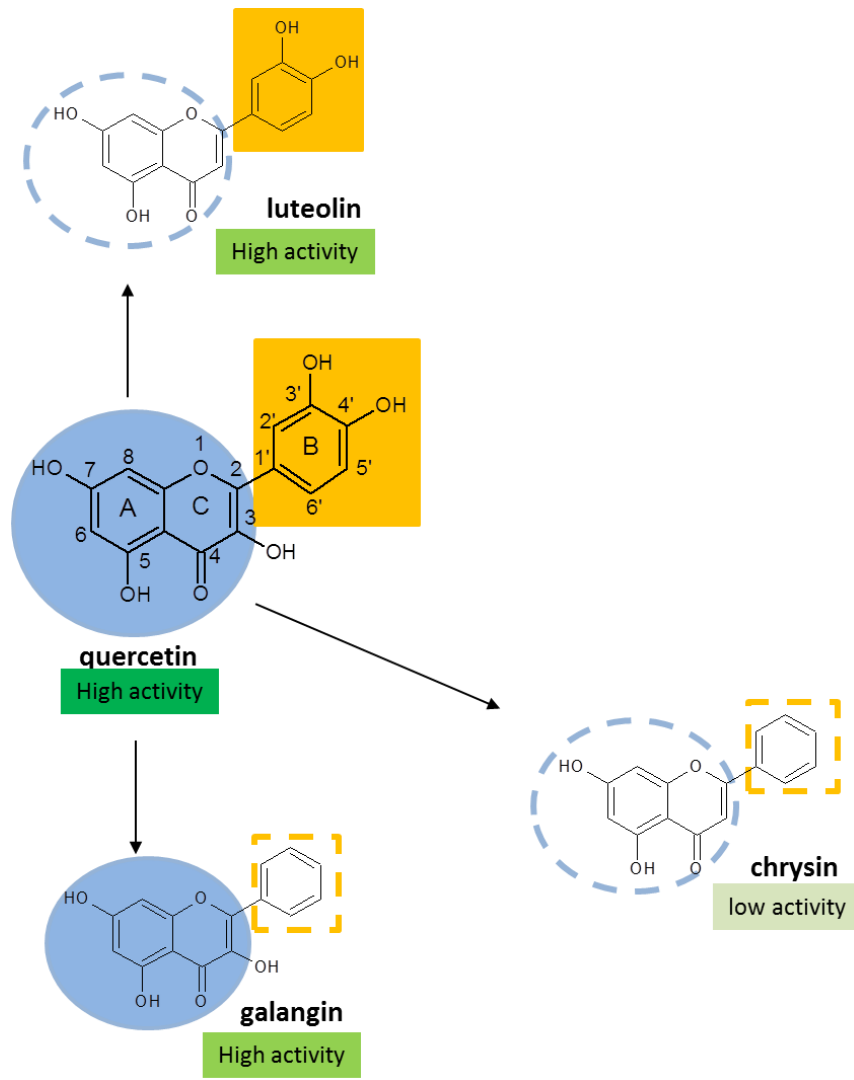

Fig. 1. Demonstration of the presence of two antioxidant pharmacophores in quercetin (Q), the AC-ring (shown in blue) and the B-ring (shown in orange). Q is a very potent antioxidant in the prevention of lipid peroxidation or the peroxynitrite scavenging. Galangin, which does not have the $3^{\prime} \mathrm{OH}$ and $4^{\prime} \mathrm{OH}$ groups, also has a high activity, demonstrating that the AC-ring of $\mathrm{Q}$ is a pharmacophore. Chrysin, without the $3 \mathrm{OH}$ group, has a much lower activity than galangin. This shows the importance of the $3 \mathrm{OH}$ group in the AC-ring pharmacophore. Luteolin that does not have the active AC-ring pharmacophore because it misses the $3 \mathrm{OH}$ group, is still a very effective antioxidant. This can be ascribed to the B-ring that is a catechol group, the other pharmacophore. These antioxidant activities are taken from Heijnen et al. [28]. 

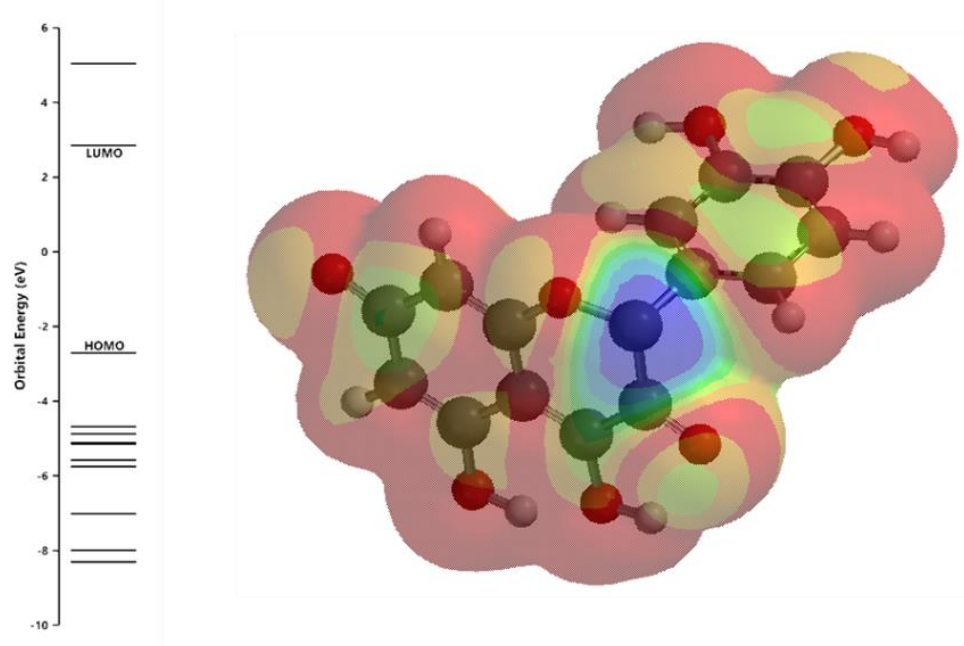

Fig. 2. The highest occupied molecular orbital (HOMO) map of the $\mathrm{Q}$ anion generated with ab initio calculations in Spartan'18. Left are the orbital energies. Right is the HOMO map of the Q anion, which is the active antioxidant of Q. Blue indicates a high concentration of the HOMO, and red indicates a low concentration. This shows that the HOMO of $\mathrm{Q}$ anion is highly concentrated at the $\mathrm{C} 2$ position.
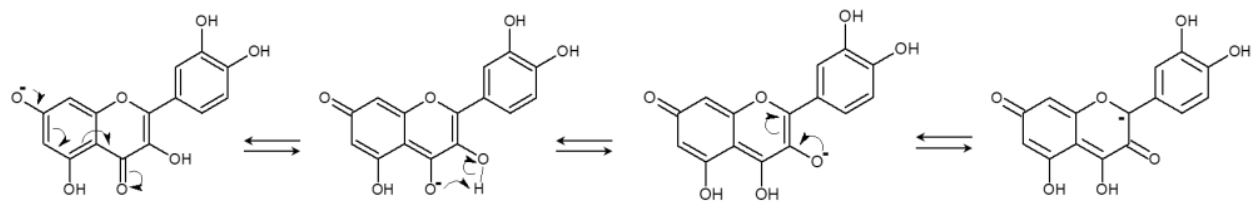

Scheme I. Stepwise illustration of how the HOMO of the Q anion can be focused on the C2 position. The $7 \mathrm{OH}$ group is the most acidic one and can be easily deprotonated at physical $\mathrm{pH}$. First, the negative charge on the oxygen at 7 position is transferred to the 4 carbonyl group via a keto-enol like tautomerization. Subsequently, the proton of the $3 \mathrm{OH}$ group is transferred to the negatively charged oxygen on the 4 position. Finally, the negative charge on the deprotonated $3 \mathrm{O}^{-}$is delocalized via the $\mathrm{C} 3=\mathrm{C} 2$ double bond to the $\mathrm{C} 2$ position.

Within Q, two pharmacophores have been identified, i.e. the AC-ring and the B-ring (Fig. 1). It is unclear that which of the two pharmacophores is primarily involved in the reaction with free radicals $[29,30]$. A positive correlation between the antioxidant activity of polyphenols and the number of $\mathrm{OH}$ groups has been found [31]. Moreover, the electron density will be the highest on the oxygen atoms because of their relative high electron negativity. Probably therefore, it is commonly assumed that the first electron is abstracted from one of the oxygen atoms in Q. However, the tendency to donate an electron is not primarily governed by electron density, it depends more on the energy of the highest molecular orbital (HOMO). The electrons in the HOMO are the most loosely bound and therefore the easiest to be donated. We did the quantum molecular calculations (QMCs) to determine where the HOMO has the highest energy. Because the $7 \mathrm{OH}$ group is deprotonated at physiological $\mathrm{pH}$, the calculations were performed on the $7 \mathrm{OH}$ 
deprotonated anion of Q [32]. Another reason to use Q anion is that Q does not act as an efficient antioxidant at low $\mathrm{pH}$ when the $7 \mathrm{OH}$ group and all the other $\mathrm{OH}$ groups are protonated $[33,34]$. QMC shows that in the HOMO map of Q anion, the HOMO is most concentrated on the $\mathrm{C} 2$ position (Fig. 1). This highlights the importance of the $\mathrm{C} 2$ position and indicates that the free radical will abstract an electron at the $\mathrm{C} 2$ position of $\mathrm{Q}$ anion.

How the HOMO can be concentrated on the C2 is depicted in Scheme II. After deprotonation, the negative charge on the oxygen at the 7 position of the $\mathrm{Q}$ anion can be channeled to the carbonyl group at the 4 position via a keto-enol like tautomerization. Subsequently, the negatively charged oxygen on the 4 position can get a proton from the $3 \mathrm{OH}$ group and create a negative charge on the oxygen at the 3 position. This negative charge is channeled to the $\mathrm{C} 2$ position via the $\mathrm{C} 3=\mathrm{C} 2$ double bond. In this process, the interaction of the $3 \mathrm{OH}$ group with the carbonyl group at the 4 position play a pivotal role in channeling the electron density from the negatively charged oxygen at the 7 positon to the C2 position.

With $\mathrm{Q}$ analogues, it was found that $\mathrm{OH}$ groups at the 3, 5 and 7 positon all contribute to the antioxidant activity of Q. However, the most important group appeared to be the $3 \mathrm{OH}$ group [29]. When the $3 \mathrm{OH}$ group is missing or substituted, the antioxidant activity is completely lost. Moreover, of the mono hydroxyl quercetin analogues, $3 \mathrm{OH}$ flavone is a far better antioxidant that $5 \mathrm{OH}$ flavone or $7 \mathrm{OH}$ flavone, as the latter two hardly display antioxidant activity. This corroborates the pivotal role of the $3 \mathrm{OH}$ group depicted in Scheme II. The $3 \mathrm{OH}$ group can directly increase the electron density on the $\mathrm{C} 2$ position. The deprotonated $5 \mathrm{OH}$ or $7 \mathrm{OH}$ group needs to interact with the carbonyl group at the 4 position to indirectly increase the electron density to the $\mathrm{C} 2$ position. In the channeling of the electron density of deprotonated $7 \mathrm{OH}$ group in $\mathrm{Q}$, the interplay of the $3 \mathrm{OH}$ group with the carbonyl group at 4 position is essential. This is in line with its pivotal role of the $3 \mathrm{OH}$ group and 4 carbonyl group in the antioxidant activity of several $\mathrm{Q}$ analogues $[28,35,36]$.

Apparently, the AC-ring is the predominant pharmacophore in Q. Compared to the Bring, the $\mathrm{AC}$-ring contains the most acidic $\mathrm{OH}$ group, and has more hydroxyl groups that all focus electron density on the $\mathrm{C} 2$ position and concentrate the HOMO there. All evidence points to the $\mathrm{C} 2$ position of $\mathrm{Q}$ where the first electron is donated to a free radical. 
Step 2: $Q$ radical $\left(Q^{*}\right)$ undergoes intramolecular scavenging to delocalize the unpaired electron (UE) in the B-ring

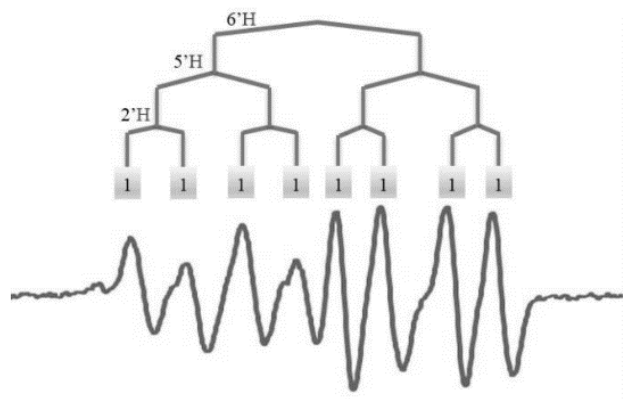<smiles></smiles>

Fig. 3. Panel A shows the ESR spectrum of the $Q$ radical $\left(Q^{\bullet}\right)$ that consists of three doublets caused by the three protons in the B-ring. The splitting constant of 6'pronton, 5'proton and 2' proton are 3,184, 1.480 and 0.652 respectively. The relative intensities of the peaks are summed up in the grey rectangles. Panel B shows the proposed structure of $\mathrm{Q} \cdot$ with the spin-distribution $(\%)$ calculated by O'Connell's equation from the hyperfine coupling constants of the ESR spectrum.

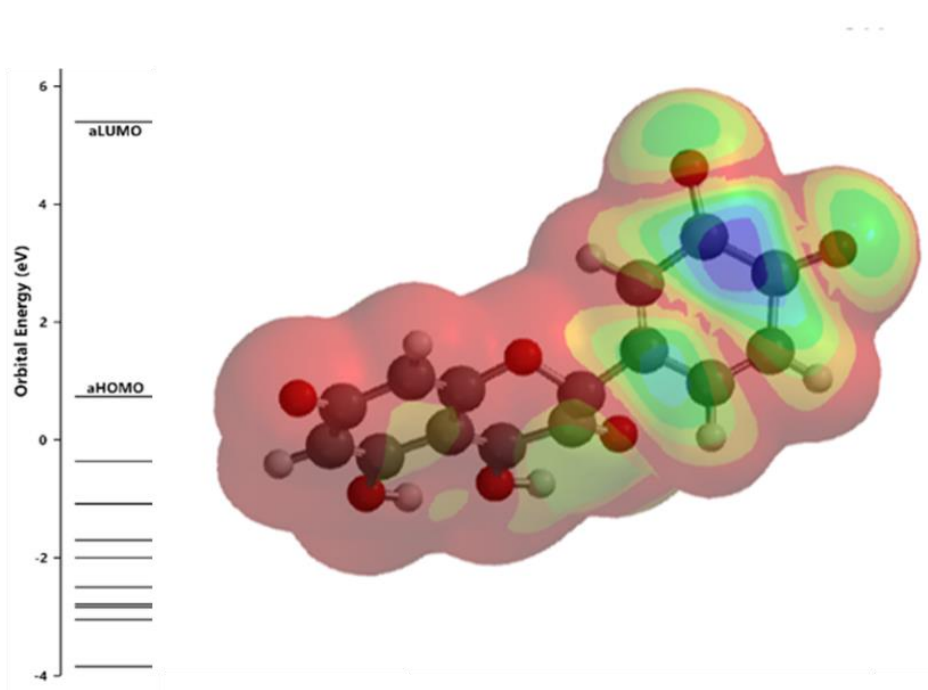

Fig. 4. The frontier orbitals of the single occupied molecular orbital (SOMO, unpaired electron) of the Q• as calculated by Spartan'18, which shows a high concentration (blue) in the B-ring of Q• 


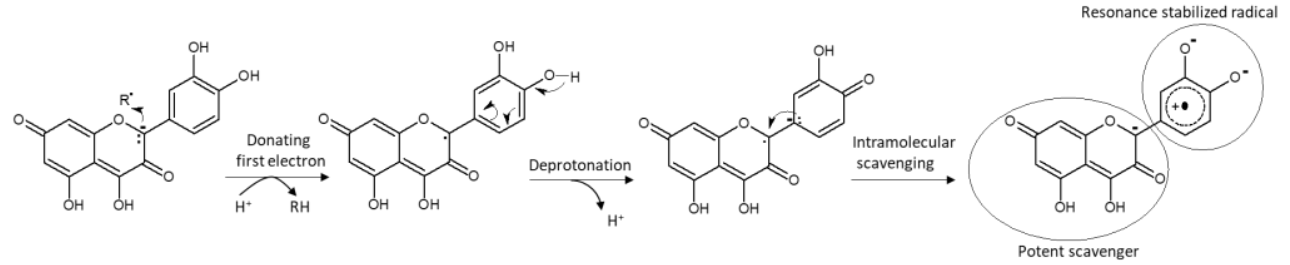

Scheme II. The proposed intramolecular scavenging of the AC-ring radical by the B-ring in Q•. Q donates one electron and becomes a radical with the unpaired electron (UE) located on C2. The UE on the C2 position is immediately scavenged by the $\mathrm{C} 1$ ' intra-molecularly. The deprotonated oxygen at the 4' position concentrates the electron density at the $\mathrm{C} 1$ ' position, thus enhances the intra-molecular scavenging of the AC-ring and B-ring. By the intramolecular scavenging, the AC-ring pharmacophore is restored to scavenge another free radical, and the $\mathrm{UE}$ is delocalized in the B-ring as found with the ESR spectrum.

After donating an electron to a free radical, $\mathrm{Q}$ is converted into a $\mathrm{Q}$ radical ( $\left.\mathrm{Q}^{*}\right)$ that has taken over part of the reactivity of scavenged free radical. The reactivity of $\mathrm{Q}^{\bullet}$ is lower than that of the radical scavenged, which is the essence of the protective effect of free radical scavenging activities. The stabilization of $\mathrm{Q}^{*}$ is caused by delocalization of the UE over the molecule. The delocalization can be measured using electron spin resonance spectroscopy (ESR). The ESR spectrum, that is caused by the spin of the UE, is influenced by the nucleus of paramagnetic atoms in the neighborhood of the UE [37]. In the ESR spectrum of $\mathrm{Q}^{*}$, the interaction of the UE with protons is seen by splitting of signal into doublets. The higher the interaction between the proton and the UE, the larger the distance between the peaks of the doublet, i.e. the larger the splitting constant (a) [38].

The ESR spectrum of Q consists of three doublets (Fig. 3). By comparing the ESR spectra of various polyphenols, it was found that the UE in $\mathrm{Q}^{\bullet}$ interacts with 3 protons, all located on the B-ring; (i) the 6' proton causes the largest, (ii) the 5' proton causes the intermediate and (iii) the 3' proton causing the smallest doublet $[39,40]$. Using O'Connel's equation, the spin density calculated from the hyperfine couplings is given in Fig. 2. Apparently, the UE is primarily delocalized in the B-ring.

QMC was used to calculate the single occupied molecular orbital (SOMO) map of $\mathrm{Q}^{\circ}$ to show the distribution of the UE within the molecule. It shows that the UE is primarily delocalized in the B-ring, which qualitatively corroborates the ESR data (Fig. 4). This seems to be in contrast to our conclusion that the AC-ring of Q donates the first electron, which would mean that $\mathrm{Q}$ becomes a radical with its UE located on the $\mathrm{C} 2$ position in the AC-ring, and not in the B-ring. This contradiction can be resolved by postulating the intramolecular scavenging shown in Scheme III. This likelihood of intramolecular scavenging is increased by the presence of the 4'OH group in Q'. The oxygen at 4' position concentrates the electron density at the $\mathrm{C} 1$ ' position, thus enhances the intra-molecular scavenging of the AC-ring. So, the $4^{\prime} \mathrm{OH}$ of $\mathrm{Q}^{\circ}$ is essential for the intra molecular scavenging of the AC-ring and the stabilization of the UE in the B-ring. This would mean that $\mathrm{Q}^{*}$ with the $\mathrm{UE}$ on the $\mathrm{C} 2$ position is a short-lived intermediate that is rapidly converted to the more stable $\mathrm{Q}^{\bullet}$ with the UE delocalized in the B-ring. 


\section{Step 3: $Q^{\bullet}$ donates a second electron at the $\mathrm{C} 2$ position to a free radical}

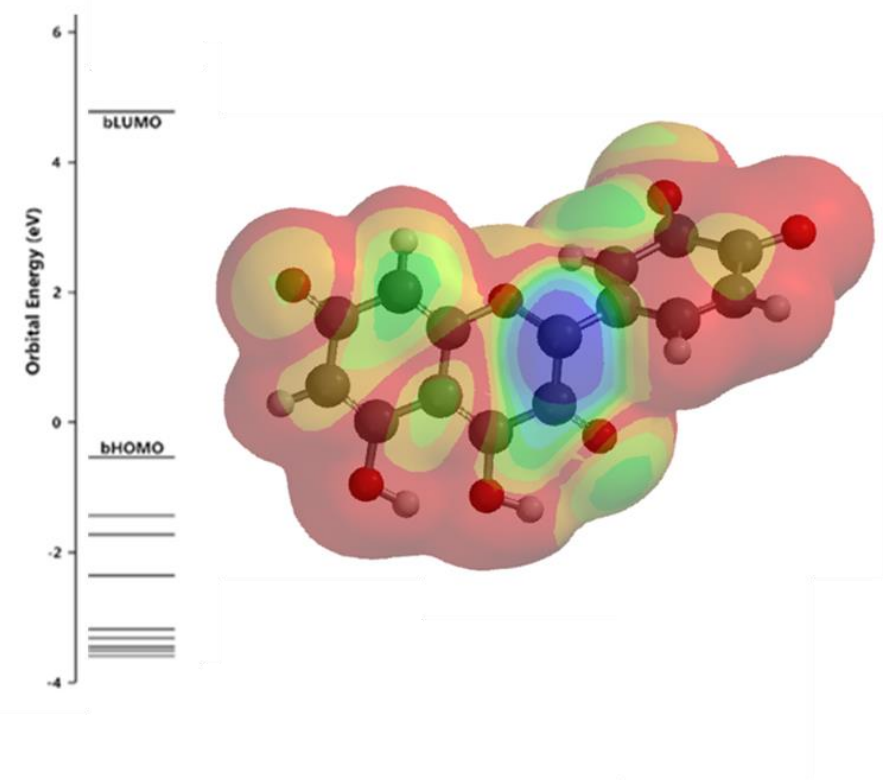

Fig. 5. The HOMO map of Q' as calculated by Spartan'18, which shows a high concentration (blue) on the C2 position. Right is the parallel orbital energies.

Due to the intra molecular radical scavenging, not only the UE of $\mathrm{Q}^{\bullet}$ is stabilized by the delocalized of UE in the B-ring, also the "original" AC-ring is restored. This creates the potential to donate a second electron to a free radical at $\mathrm{C} 2$ position (Scheme II). Fig. 5. confirms that the HOMO of $\mathrm{Q}^{-}$is mostly concentrated on the $\mathrm{C} 2$ position. This high concentration of a HOMO indicates that the second electron will also be donated from the $\mathrm{C} 2$ position. Intriguingly, QMC shows parallel energy levels for the $\mathrm{Q}^{*}$ with the SOMO concentrated in the B-ring and the HOMO concentrated on the $\mathrm{C} 2$ position. Due to a higher electron donating effect of the catechol radical in $\mathrm{Q}^{*}$ compared to the deprotonated catechol in $\mathrm{Q}, \mathrm{Q}^{*}$ is expected to donate a electron quicker than $\mathrm{Q}$. This also indicates that the ACring and B-ring are not completely independent pharmacophores. This is corroborated by the highest antioxidant activity of Q compared to that of e.g. galangin [36]. 


\section{Step 4: $Q$ quinone methide $(Q Q)$ is formed by combining of two UEs}

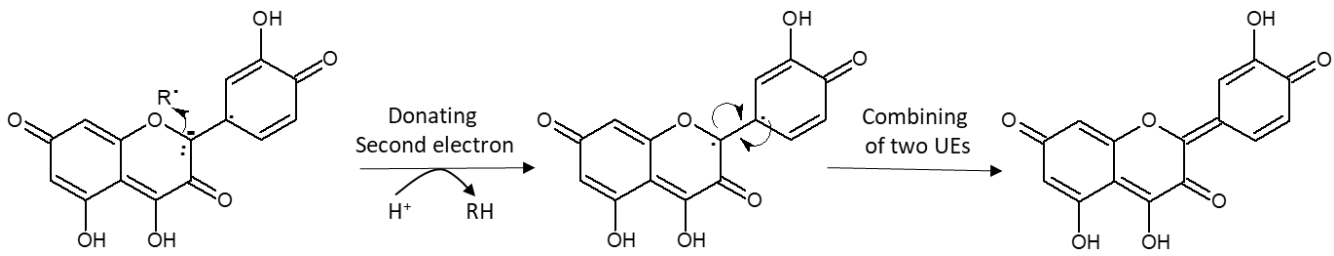

Scheme III. The proposed scavenging of the free radical by Q: The stabilized Q' can donate an electron from the $\mathrm{C} 2$ position to the free radical, which will generate a biradical with UEs one at the $\mathrm{C} 2$ position and one in the $\mathrm{B}$ ring. The two UEs combine to form an electron pair and quercetin quinone methide (QQ) is formed.

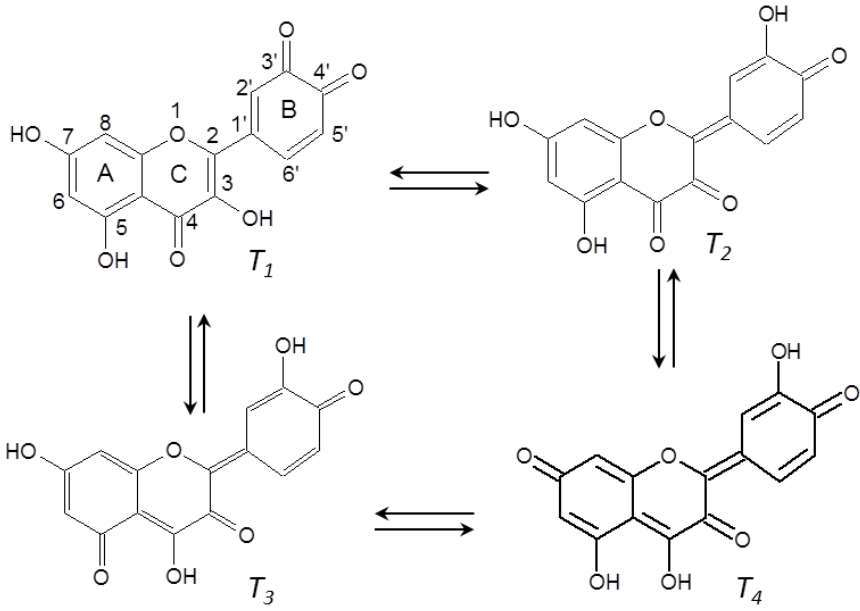

Fig. 6. Four different tautomers of QQ. $T_{4}$ has is the most favorable tautomer because the carbonyl groups are at maximum distance from each other. The relative abundance of $\mathrm{T}_{4}$ is more than $99 \%$ based on quantum molecular calculations

So $\mathrm{Q}^{\bullet}$ also donates the second electron to a free radical at the $\mathrm{C} 2$ positon. In this case, a biradical with one $\mathrm{UE}$ at the $\mathrm{C} 2$ position and one in the $\mathrm{B}$-ring will be formed. The presence of two UEs in a conjugated ring system is energetically unfavorable, forcing the two UEs to combine into an electron pair, resulting in the formation of the more stable quercetin quinone methide (QQ) (scheme III). 
There are four possible tautomers of QQ as illustrated in Fig. 6 [41]. The quantum molecular calculations reveal that $\mathrm{T}_{4}$ is the most favorable one with reactive abundance of more than $99 \%$. In $\mathrm{T}_{4}$, the distance between the electron rich oxygen of the carbonyls attached to the AC-ring as well as the B-ring is maximal, which explains why it is energetically favorable and therefore has the highest abundance.

\section{Step 5: QQ can react with ascorbate, GSH and water}
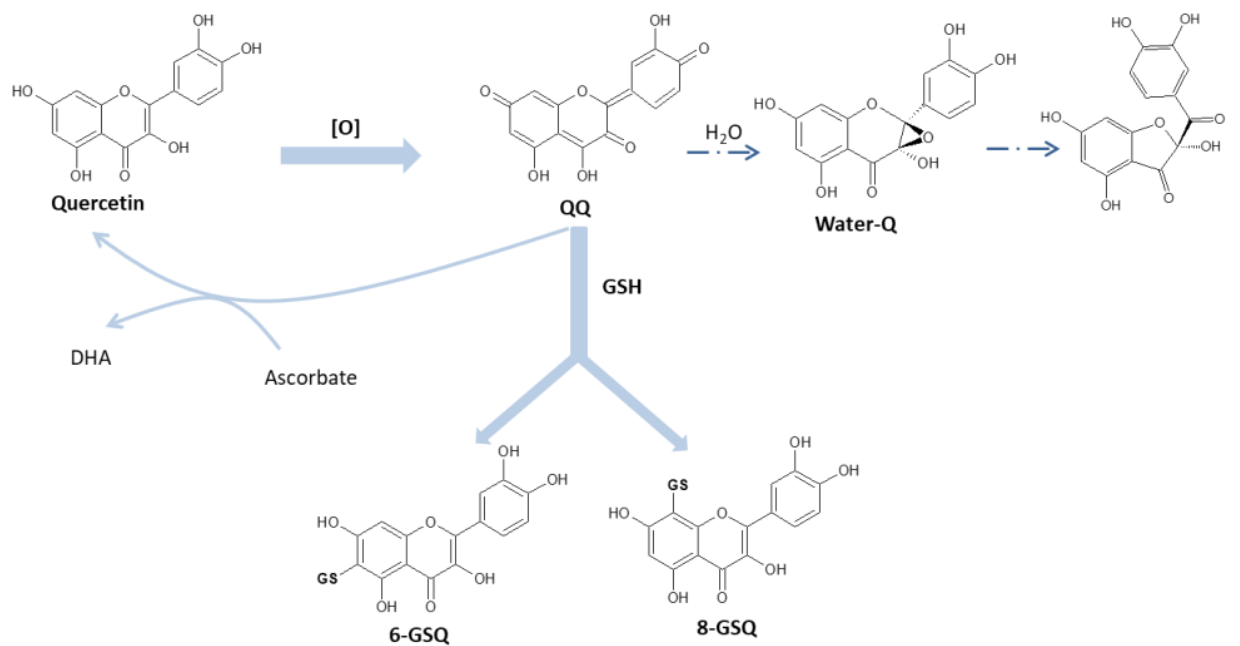

Fig. 7. Interplay of QQ with ascorbate, GSH and water. When Q is oxidized, QQ can be formed. Ascorbate can recycle QQ to Q, during which ascorbate becomes oxidized giving DHA (dehydroascorbate). QQ can also react with GSH, thereby forming 6-GSQ and 8-GSQ. QQ reacts much faster with GSH than with ascorbate, and is less likely react with water to form the water-Q adduct. 


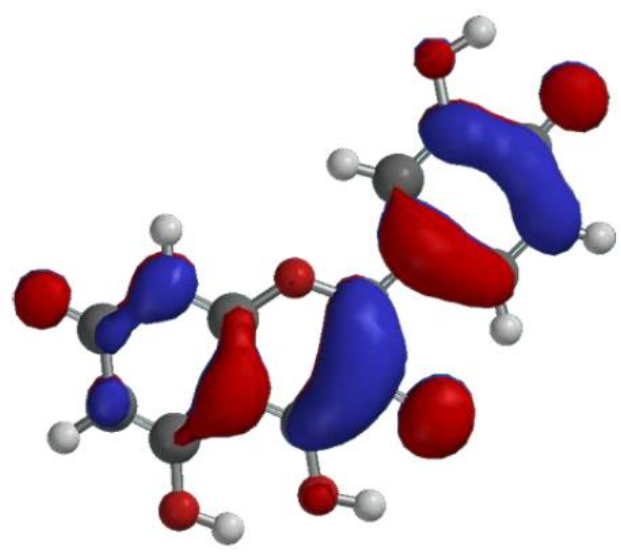

Fig. 8. Delocalization of the lowest unoccupied molecular orbital (LUMO) of QQ, which shows the lowest enery place at 6 and 8 positon of AC-ring.

In the antioxidant network, QQ can be recycled to $\mathrm{Q}$ by ascorbate in a redox reaction or reacts with GSH in a Michael addition [42] (Fig. 7). It appears that the reaction of QQ with GSH is much quicker than that of QQ with ascorbate. This difference can be explained by Pearson's hard-soft-acid-base concept. The theory is that "soft" electrophiles react quicker with "soft" nucleophiles, whereas "hard" electrophiles react quicker with "hard" nucleophiles. "Hard" electrophiles are chemical species that are small, highly polarized, and have a high oxidation state. Thiols such as GSH are known to be "softer" than ascorbate $[41,43,44]$. Since QQ prefers to react with the "soft" GSH over the "hard" ascorbate, QQ should be clasified as "soft". This is in line with the distribution of the LUMO of QQ that spreads all over the entire molecule (Fig. 8), and that GSH adducts to the 6 or 8 position where the LUMO of QQ is relatively low.

Besides ascorbate and GSH, QQ can react also with water and form the water-Q adduct. That water is a relatively hard compound compared to GSH and ascorbate explains why the reaction of the relatively soft QQ with either GSH or ascorbate is much quicker than the reaction of QQ with water. The reaction of QQ with water can be neglected when either GSH or ascorbate is present $[41,45]$. Nevertheless, QQ has a relative short half life in water. Trapping of QQ by the formation of GSH adducts was the best (indirect) prove to show that QQ is formed as a reactive intermediate. Recently, we were able to detect QQ during the oxidation of Q using UHPLC-ESI-Q-TOF/MS (supplemental data) that provides the direct prove for the formation of QQ.

The reaction of polyphenolic quinones with thiols can also be involved in the indirect antioxidant activity of polyphenols such as Q. Thiol groups on KEAP1 can be arylated by the polyphenolic quinones. The arylated KEAP1 will dissociate from its complex with Nuclear factor erythroid 2-related factor 2 (Nrf2). Subsequently, the freed Nrf2 will translocate to the nucleus and stimulate the transcription of numerous detoxifying and antioxidant genes. This will strengthen the defense and increase the resilience of the cell 
$[46,47]$. Based on its chemical reactivity, QQ is expected to react with the thiol group of KEAP1 and activate the Nrf2 pathway more efficiently than free radicals. This is in line with reports on the induction of the endogenous antioxidant defense by Q. However, attempts to detect the KEAP1-Q adduct using the same protocol used in successfully detecting the KEAP1-monoHER adduct failed [27]. This might due to the instability of the Michael adduct of thiols with QQ. Possibly in the process of analyzing, the KEAP1-Q adduct dissociates. So despite the likeliness of this redox modulating effect of $\mathrm{Q}$, direct evidence has not been produced.

\section{Discussion}

We addressed the redox modulating effect of Q. It should be noted that apart from their redox modulating effects, polyphenols also display other biological activities. Polyphenols can form strong non-covalent interactions (hydrogen bonding, ionic and hydrophilic interactions). Consequently, they can chelate various metals and bind with practically all proteins and are able to bind at more than one point of a protein surface. This explains why polyphenols have been shown to inhibit a wide range of enzymes, including radical generating enzymes and antioxidant enzymes. However, no specific "polyphenol receptor" has been found. Paradoxically, due to their reducing capacity, polyphenols can also donate electrons to oxygen leading to the formation of reactive oxygen species. In general, these additional reactions are seen at a concentration above concentrations that are normally reached in vivo, which indicates that these reactions are not relevant for the physiological effects of polyphenols. Nevertheless, small aspecific inhibition of many enzymes will slow down cell activity and this might significantly reduce radical formation [48-50]. A huge variation in these "aspecific effects" of Q and other polyphenols have been reported, making it impossible to draw a general conclusion.

Also in the redox modulating effects, there are a lot of differences between polyphenols. By comparing quercetin and (-)-epicatechin, it was found that the LUMO of QQ is spread all over the molecule, which is possibly because of the small dihedral angle between the AC-ring and B-ring. For (-)-epicatechin, the LUMO of (-)-epicatechin quinone is concentrated in the B-ring, which corroborates with the relatively large dihedral angle between the AC-ring and B-ring. In presence of GSH and ascorbate, QQ preferentially passed its reactivity to GSH, but (-)-epicatechin reacts faster with ascorbate $[25,42]$. Comparing their LUMO maps indicates that (-)-epicatechin is harder than Q. This difference in soft/hardness between polyphenols will result in differences in redox modulating effects as well as therapeutic effects of both polyphenols, which needs to be further explored. 


\section{Overview}

Our systematic approach has better unraveled the various steps involved in the redox modulating activity of $\mathrm{Q}$ and has showed how the various functional groups cooperate with each other.

Firstly, Q will donate an electron to a free radial from the $\mathrm{C} 2$ position. In the $\mathrm{Q}$, the UE is transformed by intramolecular scavenging from the $\mathrm{C} 2$ position to the $\mathrm{B}$-ring, and the UE is stabilized in the B-ring. The $\mathrm{Q}^{\bullet}$ will donate a second electron to a free radical also from the C2 position. The $\mathrm{Q}^{\bullet}$ formed is quickly transformed into QQ. The QQ has a soft character and might induce cellular protection, however the proposed molecular mechanism has not been provided. An overview of the relative energies of the different Q-species in the radical scavenging is given in Fig. 9. How the various functional groups cooperate with each other is shortly described in the legend of Fig. 9.

The systematic unravelling of the redox modulating of $\mathrm{Q}$ on molecular level gives insight in the antioxidant activity of polyphenols. This forms the fundament to select the appropriate redox modulating compound to protect against a specific redox mediated disorder. 


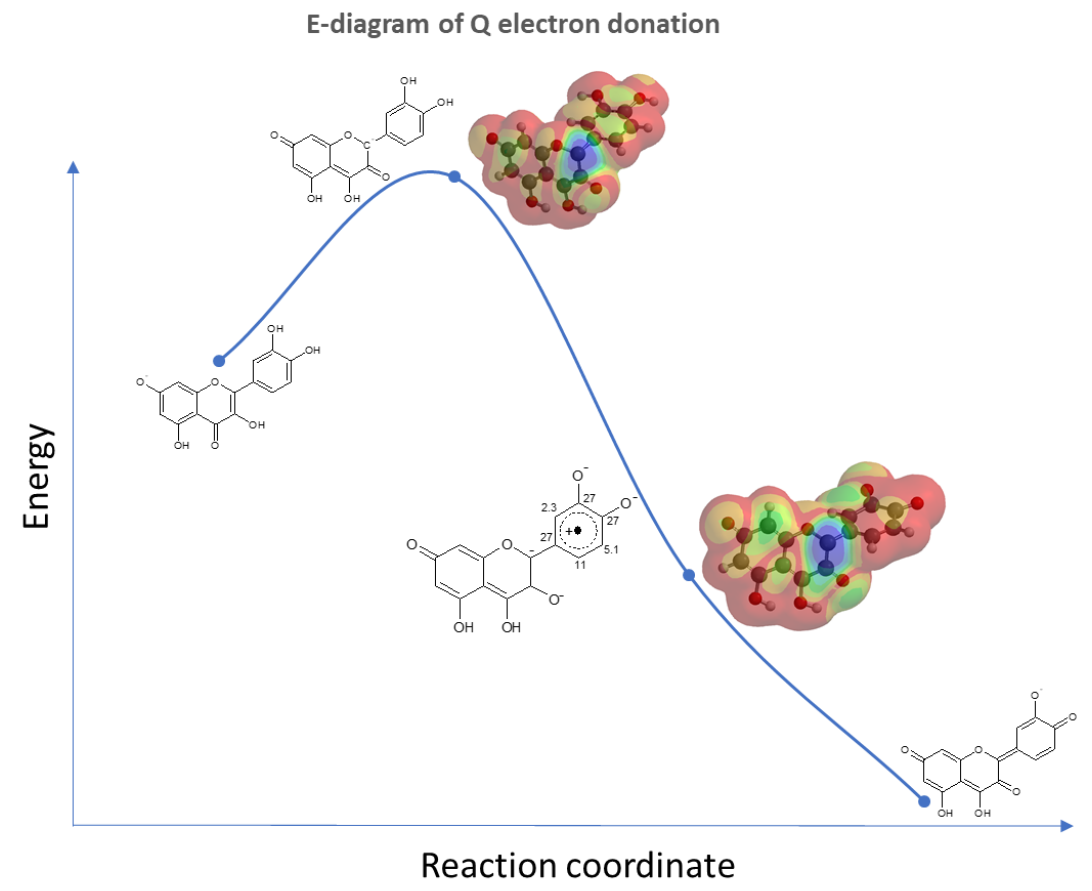

Fig. 9. The energies of the different molecular species of $Q$ during radical scavenging. Q will donate an electron to a free radical from the $\mathrm{C} 2$ position, since the $\mathrm{HOMO}$ of $\mathrm{Q}$ concentrates on the $\mathrm{C} 2$ position of the $\mathrm{AC}$-ring pharmacophores. The electron density at $\mathrm{C} 2$ is directly increased by the $3 \mathrm{OH}$ group. The 5 and $7 \mathrm{OH}$ groups can interact with $3 \mathrm{OH}$ group via keto-enol like tautomerization and indirectly increase the electron density at $\mathrm{C} 2$. In this channeling of the electron density of $\mathrm{C} 2$, the interplay of the $3 \mathrm{OH}$ group with the carbonyl group at the $\mathrm{C} 4$ position is essential. After donating the electron to free radicals, Q is oxidized into Q'. Q donates an electron at the $\mathrm{C} 2$ position, then the UE is located on C2. However, this UE at C2 can be immediately scavenged by the C1' intra-molecularly. The oxygen at 4' position concentrates the HOMO at the C1' position and thus enhances the intra-molecular scavenging of the AC-ring. ESR spectrum shows that the UE of Q' is delocalized and stabilized in the B-ring. In the intra molecular scavenging of the AC-ring by the B-ring, the AC-ring is restored with the highest electron density on $\mathrm{C} 2$ position again. This enables the stabilized $\mathrm{Q}^{*}$ to donate a second electron to free radicals from C2 and becomes the two-electron oxidized product, QQ. QQ is stabilized by its own tautomerization is the most stable and most abundant one. In the antioxidant network, QQ can be either recycled to Q by ascorbate or forms GSH-Q adducts with GSH. Moreover, QQ can strengthen the defense system of an organism indirectly by reacting with thiols on KEAP1, but no KEAP1-Q adduct has been detected. 


\section{Molecular quantum calculations}

QMCs were performed in the gas phase at the ground state using Spartan'18. Energies for the HOMO map of Q anion and $\mathrm{Q}^{*}$, and the SOMO map of $\mathrm{Q}^{*}$ were calculated with the density functional theory (DFT) $\omega$ B97X-D using the 6-31G* basis set [20]. The equilibrium geometry of the QQ was optimized with the semi-empirical method PM3, and its energy for the generation of its LUMO map was calculated with the DFT $\omega$ B97X-D using the $6-31 \mathrm{G}^{*}$ basis set. The equilibrium geometry of $\mathrm{Q}^{-}$was optimized with the semi-empirical method PM3, and its energy was calculated with the DFT $\omega$ B97X-D using the 6-31G* basis set.

\section{Electron Spin Resonance}

The ESR spectra were recorded on a Bruker ESP300 ESR spectrometer. Modulation frequency was $100 \mathrm{kHz}$, the amplitude $1 \mathrm{G}$. Microwave frequency was set at $9.79 \mathrm{GHz}$, with a power of $20 \mathrm{~mW}$. Magnetic field sweep widths were in the range 0.9-2.0 mT, depending on the spectral widths. A modulation amplitude of $0.01 \mathrm{mT}$ was used, and the spectrum was accumulated in ten scans. After placing the flat cell in the microwave cavity, the spectrometer was tuned manually to minimize the time between start of the reaction and recording the spectrum. The hyperfine couplings (a) observed in the ESR spectrum were used to calculate the spin densities $(\rho \pi)$ on the carbons according to O'Connell's equation: $\mathrm{a}=29^{*} \rho \pi$. Q was dissolved in DMSO to a concentration of 1-5 mM. $250 \mu \mathrm{L}$ of $0.1-1 \mathrm{M}$ solution of $\mathrm{NaOH}$ (aq) was added to $650 \mu \mathrm{L}$ of the quercetin solution in a flat cell [51]. 


\section{Supplementary material}
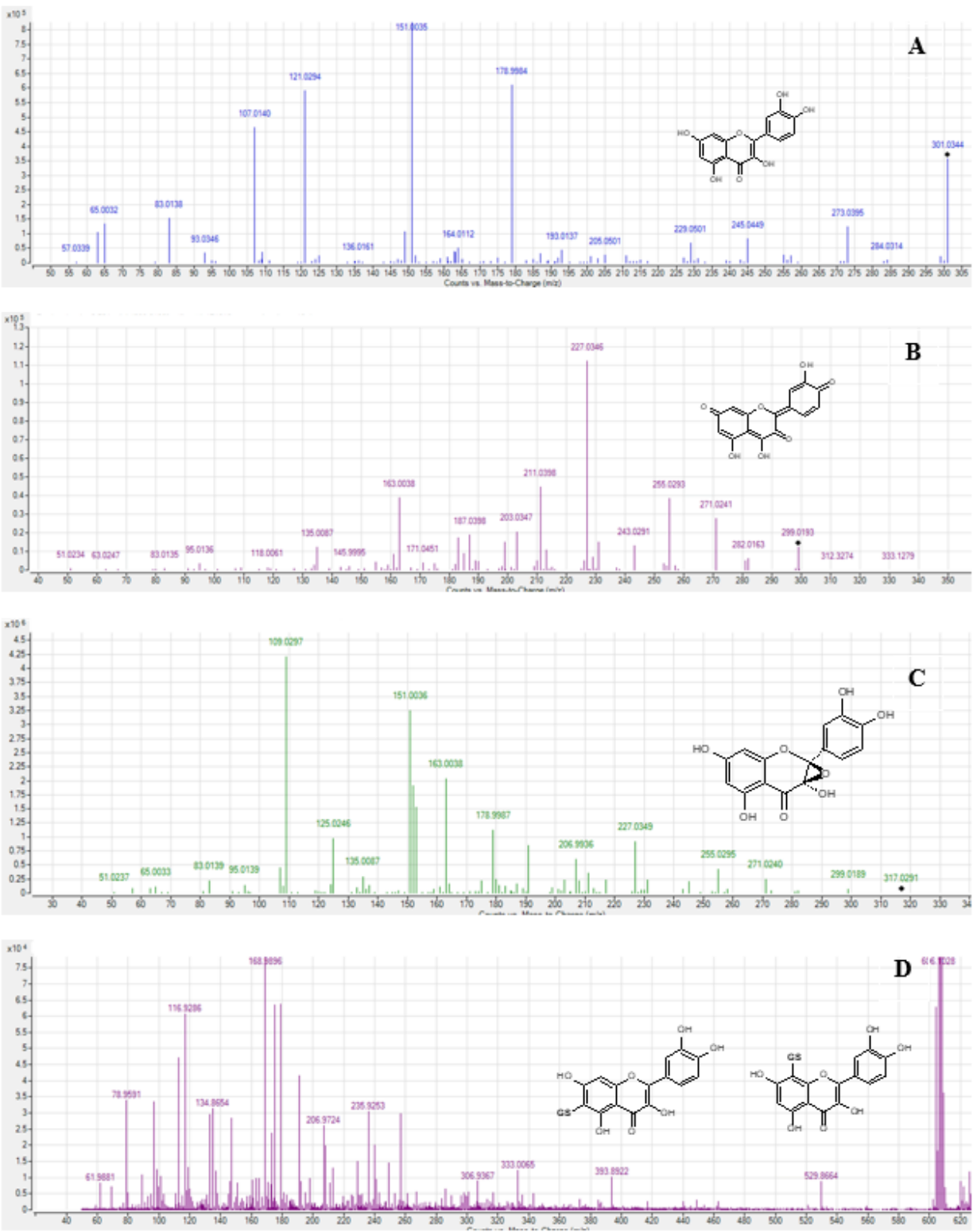

Fig. 1. LC/MS-MS spectra of $50 \mu \mathrm{M}$ quercetin (A), quercetin quinone (B), quercetin-water (C) and quercetinGSH (D) with their relative proposed fragmentations 
To get direct evidences for quercetin and its oxidized products, the Q-TORF MS/MS spectra of Q, QQ, water-Q and GSQ adducts were detected. Quercetin yielded fragment ions at $151.0 \mathrm{~m} / \mathrm{z}, 121.0 \mathrm{~m} / \mathrm{z}$ and $179.0 \mathrm{~m} / \mathrm{z}$. The protonated and fragmented ions of QQ are $227.0 \mathrm{~m} / \mathrm{z}$ and $211.0 \mathrm{~m} / \mathrm{z}$. the water-Q has fragments at $198.0 \mathrm{~m} / \mathrm{z}, 151.0 \mathrm{~m} / \mathrm{z}$ and $163.0 \mathrm{~m} / \mathrm{z}$. The characteristic fragment ions of GSQ are $169.0 \mathrm{~m} / \mathrm{z}$ and $117.0 \mathrm{~m} / \mathrm{z}$. It is concluded that quercetin is first converted into QQ, and subsequently QQ reacts with GSH into GSH adducts. QQ can be regenerated into quercetin by ascorbate. Meanwhile, QQ can react with water to form a water-Q adduct. 


\section{References}

1. Kohen, R.; Nyska, A. Oxidation of biological systems: oxidative stress phenomena, antioxidants, redox reactions, and methods for their quantification. Toxicologic pathology 2002, 30, 620-650.

2. Diplock, A.; Charuleux, J.L.; Crozier-Willi, G.; Kok, F.; Rice-Evans, C.; Roberfroid, M.; Stahl, W.; VinaRibes, J. Functional food science and defence against reactive oxidative species. British journal of nutrition 1998, 80, S77-S112.

3. Chaudiere, J.; Ferrari-Iliou, R. Intracellular antioxidants: from chemical to biochemical mechanisms. Food and chemical toxicology 1999, 37, 949-962.

4. Lyakhovich, V.V.; Vavilin, V.A.; Zenkov, N.K.; Menshchikova, E.B. Active defense under oxidative stress. The antioxidant responsive element. Biochemistry. Biokhimiia 2006, 71, 962-974.

5. Haenen, G.R.; Veerman, M.; Bast, A. Reduction of beta-adrenoceptor function by oxidative stress in the heart. Free radical biology and medicine 1990, 9, 279-288.

6. Haenen, G.R.; Plug, H.J.; Vermeulen, N.P.; Timmerman, H.; Bast, A. Contribution of 4-hydroxy-2,3-transnonenal to the reduction of beta-adrenoceptor function in the heart by oxidative stress. Life sciences 1989 , 45, 71-76.

7. Bast, A.; Kaiserova, H.; den Hartog, G.J.; Haenen, G.R.; van der Vijgh, W.J. Protectors against doxorubicininduced cardiotoxicity: flavonoids. Cell biology and toxicology 2007, 23, 39-47.

8. Kooy, N.W.; Royall, J.A.; Ischiropoulos, H.; Beckman, J.S. Peroxynitrite-mediated oxidation of dihydrorhodamine 123. Free radical biology and medicine 1994, 16, 149-156.

9. Rietjens, S.J.; Bast, A.; Haenen, G.R. New insights into controversies on the antioxidant potential of the olive oil antioxidant hydroxytyrosol. J Agr Food Chem 2007, 55, 7609-7614.

10. Heim, K.E.; Tagliaferro, A.R.; Bobilya, D.J. Flavonoid antioxidants: chemistry, metabolism and structureactivity relationships. The Journal of nutritional biochemistry 2002, 13, 572-584.

11. Boots, A.W.; Haenen, G.R.; Bast, A. Oxidant metabolism in chronic obstructive pulmonary disease. The European respiratory journal. Supplement 2003, 46, 14s-27s.

12. Galadari, S.; Rahman, A.; Pallichankandy, S.; Thayyullathil, F. Reactive oxygen species and cancer paradox: To promote or to suppress? Free radical biology and medicine 2017, 104, 144-164.

13. Nichenametla, S.N.; Taruscio, T.G.; Barney, D.L.; Exon, J.H. A review of the effects and mechanisms of polyphenolics in cancer. Critical reviews in food science and nutrition 2006, 46, 161-183.

14. Moalin, M.; Van Strijdonck, G.P.; Beckers, M.; Hagemen, G.J.; Borm, P.J.; Bast, A.; Haenen, G.R. A planar conformation and the hydroxyl groups in the $\mathrm{B}$ and $\mathrm{C}$ rings play a pivotal role in the antioxidant capacity of quercetin and quercetin derivatives. Molecules 2011, 16, 9636-9650.

15. Cao, G.; Sofic, E.; Prior, R.L. Antioxidant and prooxidant behavior of flavonoids: structure-activity relationships. Free Radical Biology and Medicine 1997, 22, 749-760.

16. Lemmens, K.J.; Herst, P.M.; Housmans, B.A.; Moalin, M.; van der Vijgh, W.J.; Bast, A.; Haenen, G.R. The contribution of the major metabolite 4'-O-methylmonoHER to the antioxidant activity of the flavonoid monoHER. Chemico-biological interactions 2015, 239, 146-152.

17. Heijnen, C.G.; Haenen, G.R.; Minou Oostveen, R.; Stalpers, E.M.; Bast, A. Protection of flavonoids against lipid peroxidation: the structure activity relationship revisited. Free Radical Research 2002, 36, 575-581.

18. Halliwell, B. Antioxidants in human health and disease. Annual review of nutrition 1996, 16, 33-50.

19. Halliwell, B.; Gutteridge, J.M.; Aruoma, O.I. The deoxyribose method: a simple "test-tube" assay for determination of rate constants for reactions of hydroxyl radicals. Analytical biochemistry 1987, 165, 215 219.

20. Bast, A.; Haenen, G.R. The toxicity of antioxidants and their metabolites. Environ Toxicol Phar 2002, 11, 251-258.

21. Burton, G.W.; Doba, T.; Gabe, E.; Hughes, L.; Lee, F.; Prasad, L.; Ingold, K.U. Autoxidation of biological molecules. 4. Maximizing the antioxidant activity of phenols. Journal of the American chemical society 1985, 107, 7053-7065.

22. Boots, A.W.; Haenen, G.R.; den Hartog, G.J.; Bast, A. Oxidative damage shifts from lipid peroxidation to thiol arylation by catechol-containing antioxidants. Biochimica et Biophysica Acta (BBA)-Molecular and Cell Biology of Lipids 2002, 1583, 279-284.

23. Bast, A.; Haenen, G.R.; Doelman, C.J. Oxidants and antioxidants: state of the art. The American journal of medicine 1991, 91, 2S-13S.

24. Packer, J.E.; Slater, T.; Willson, R. Direct observation of a free radical interaction between vitamin E and vitamin C. Nature 1979, $278,737$.

25. Zhang, M.; Vervoort, L.; Moalin, M.; Mommers, A.; Douny, C.; den Hartog, G.J.; Haenen, G.R. The chemical reactivity of (-)-epicatechin quinone mainly resides in its B-ring. Free radical biology and medicine 2018. 
26. Lemmens, K.J.; van de Wier, B.; Vaes, N.; Ghosh, M.; van Zandvoort, M.A.; van der Vijgh, W.J.; Bast, A.; Haenen, G.R. The flavonoid 7-mono-O-( $\beta$-hydroxyethyl)-rutoside is able to protect endothelial cells by a direct antioxidant effect. Toxicology in vitro 2014, 28, 538-543.

27. Lemmens, K.J.; Sthijns, M.M.; van der Vijgh, W.J.; Bast, A.; Haenen, G.R. The antioxidant flavonoid monoHER provides efficient protection and induces the innate Nrf2 mediated adaptation in endothelial cells subjected to oxidative stress. PharmaNutrition 2014, 2, 69-74.

28. Heijnen, C.G.; Haenen, G.R.; Vekemans, J.A.; Bast, A. Peroxynitrite scavenging of flavonoids: structure activity relationship. Environmental toxicology and pharmacology 2001, 10, 199-206.

29. Trouillas, P.; Marsal, P.; Siri, D.; Lazzaroni, R.; Duroux, J.L. A DFT study of the reactivity of OH groups in quercetin and taxifolin antioxidants: the specificity of the 3-OH site. Food chemistry 2006, 97, 679-688.

30. Ohta, K. A handy way to find radical orbitals buried in UHF wavefunctions. Journal of molecular structure: THEOCHEM 2002, 587, 33-41.

31. Arora, A.; Nair, M.G.; Strasburg, G.M. Structure-activity relationships for antioxidant activities of a series of flavonoids in a liposomal system. Free radical biology and medicine 1998, 24, 1355-1363.

32. Álvarez-Diduk, R.N.; Ramírez-Silva, M.T.; Galano, A.; Merkoçi, A. Deprotonation mechanism and acidity constants in aqueous solution of flavonols: a combined experimental and theoretical study. The Journal of physical chemistry B 2013, 117, 12347-12359.

33. Musialik, M.; Kuzmicz, R.; Pawłowski, T.S.; Litwinienko, G. Acidity of hydroxyl groups: an overlooked influence on antiradical properties of flavonoids. The Journal of organic chemistry 2009, 74, 2699-2709.

34. Lemańska, K.; Szymusiak, H.; Tyrakowska, B.; Zieliński, R.; Soffers, A.E.; Rietjens, I.M. The influence of $\mathrm{pH}$ on antioxidant properties and the mechanism of antioxidant action of hydroxyflavones. Free radical biology and medicine 2001, 31, 869-881.

35. Haenen, G.R.; Paquay, J.B.; Korthouwer, R.E.; Bast, A. Peroxynitrite scavenging by flavonoids. Biochemical and biophysical research communications 1997, 236, 591-593.

36. Heijnen, C.; Haenen, G.; Van Acker, F.; Van der Vijgh, W.; Bast, A. Flavonoids as peroxynitrite scavengers: the role of the hydroxyl groups. Toxicology in vitro 2001, 15, 3-6.

37. Wertz, J. Electron spin resonance: elementary theory and practical applications, $1^{\text {st }}$ ed.; Springer Science \& Business Media: New York, US, 2012.

38. van Acker, S.A.; de Groot, M.J.; van den Berg, D.J.; Tromp, M.N.; Donné-Op den Kelder, G.; van der Vijgh, W.J.; Bast, A. A quantum chemical explanation of the antioxidant activity of flavonoids. Chemical research in toxicology 1996, 9, 1305-1312.

39. Pirker, K.F.; Stolze, K.; Pirker, K.F.; Stolze, K.; Reichenauer, T.G.; Pirker, K.F.; Stolze, K.; Reichenauer, T.G.; Nohl, H.; Pirker, K.F. Are the biological properties of kaempferol determined by its oxidation products? Free radical research 2006, 40, 513-521.

40. Kuhnle, J.; Windle, J.; Waiss, A. Electron paramagnetic resonance spectra of flavonoid anion-radicals. Journal of the chemical society B: Physical organic 1969, 613-616.

41. Moalin, M.; van Strijdonck, G.P.; Bast, A.; Haenen, G.R. Competition between ascorbate and glutathione for the oxidized form of methylated quercetin metabolites and analogues: tamarixetin, 4' O-methylquercetin, has the lowest thiol reactivity. Journal of agricultural and food chemistry 2012, 60, 9292-9297.

42. Boots, A.W.; Kubben, N.; Haenen, G.R.; Bast, A. Oxidized quercetin reacts with thiols rather than with ascorbate: implication for quercetin supplementation. Biochemical and biophysical research communications 2003, 308, 560-565.

43. Pearson, R.G. Hard and soft acids and bases. Journal of the American chemical society 1963, 85, 3533-3539.

44. Chattaraj, P.K.; Schleyer, P.v.R. An ab initio study resulting in a greater understanding of the HSAB principle. Journal of the American chemical society 1994, 116, 1067-1071.

45. Zhou, A.; Kikandi, S.; Sadik, O.A. Electrochemical degradation of quercetin: Isolation and structural elucidation of the degradation products. Electrochemistry communications 2007, 9, 2246-2255.

46. Lyakhovich, V.; Vavilin, V.; Zenkov, N.; Menshchikova, E. Active defense under oxidative stress. The antioxidant responsive element. Biochemistry (Moscow) 2006, 71, 962-974.

47. Lo, S.C.; Li, X.; Henzl, M.T.; Beamer, L.J.; Hannink, M. Structure of the Keap1: Nrf2 interface provides mechanistic insight into Nrf2 signaling. The EMBO journal 2006, 25, 3605-3617.

48. Spencer, C.M.; Cai, Y.; Martin, R.; Gaffney, S.H.; Goulding, P.N.; Magnolato, D.; Lilley, T.H.; Haslam, E. Polyphenol complexation-some thoughts and observations. Phytochemistry 1988, 27, 2397-2409.

49. Rohn, S.; Rawel, H.M.; Kroll, J. Antioxidant activity of protein-bound quercetin. Journal of agricultural and food chemistry 2004, 52, 4725-4729.

50. Häckl, L.; Cuttle, G.; Dovichi, S.S.; Lima-Landman, M.; Nicolau, M. Inhibition of angiotensin-converting enzyme by quercetin alters the vascular response to bradykinin and angiotensin I. Pharmacology 2002, 65, 182-186. 


\section{Chapter 5}

51. Bast, A.; Haenen, G.R. The toxicity of antioxidants and their metabolites. Environmental toxicology and pharmacology 2002, 11, 251-258. 


$$
\text { 的承 }
$$





\section{Chapter 6}

\section{General discussion and summary}

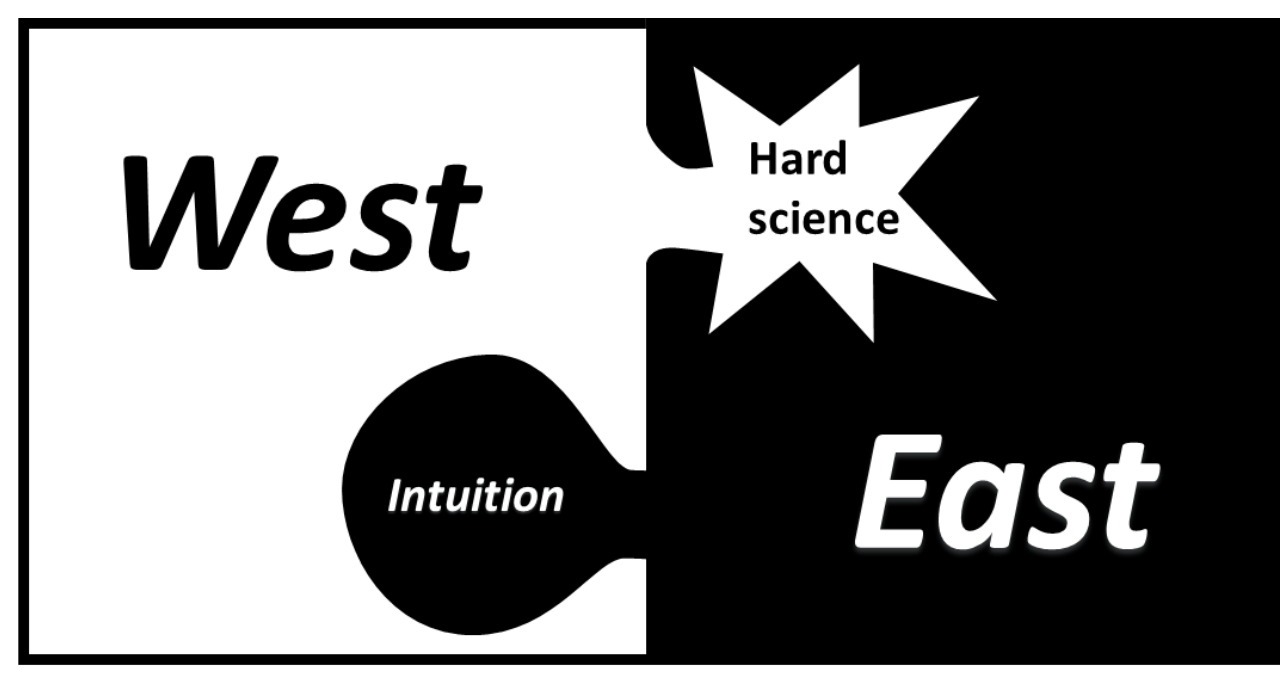


The central theme of the thesis is how to make the connection between Western and Eastern medicine. In order to do so, we should start to look at their similarities. Chapter 1 reviews the similarity between Western medicine and Eastern medicine from an energy perspective. Although Western and Eastern medicine have developed and are applied in different cultures, which explains why their therapeutic concepts are different, we found striking similarities between them. In both worlds, opposing forces provide the energy that flows through networks in an organism, which fuels life. The ability to maintain the balance between these opposing forces, i.e. homeostasis (West) and harmony (East) gives "health" and creates resilience. Moreover, strategies used to treat diseases are strikingly alike. Redox modulating compounds and TCM are added to increase or strengthen connections in the networks to finally adjust the flow of energy and regain harmony. The energy perspective provides a basis to integrate Eastern and Western medicine and results in the researches described in other chapters of the thesis.

In TCM, herbs are combined in the proper ratio to increase therapeutic effects and to reduce side effects. However, when TCM is used in the West, often only the herb with the most active ingredients is used. By the incorrect use, TCM is likely to give side effects. This is exemplified in Chapter 2 by the cardiovascular side effects of Ma Huang observed in the West. We found that the contractile response on placental arteries by Ma Huang in isolation is much higher than that of Ma Huang in combination with the other herbs in Ma Huang Decotion (MHD) as it used in TCM. This indicates that the interplay of the various compounds in MHD mitigate the side effects of Ma Huang. Previous studies showed that the other herbs in MHD also increase the therapeutic efficacy of Ma Huang. This confirmed that cardiovascular side effects reported in the West resulted from the improper use of Ma Huang in isolation, also without the supervision of knowledgeable professionals. To fully value its benefit, TCM should be used properly as developed over centuries in China.

Especially, combination of drugs might causes serious side effects in the West. For example, many Western drugs show some affinity for the acetylcholine receptors in the brain. When these drugs are combined, this may give substantial blocking of these receptors, which is known as anticholinergic accumulation (AA). Some bioactives in Traditional Chinese Medicine (TCM) are known to block acetylcholine receptors and thus potentially cause AA. In chapter 3, the AA of TCM was screened by quantifying the displacement of $\left[{ }^{3} \mathrm{H}\right]$ pirenzepine on acetylcholine receptors in a rat brain homogenate. We used a new unit to express AA, namely the Total Atropine Equivalents (TOAT). It was found that the TOAT of various herbs used in TCM was very diverse but relatively low and even negative for some herbs. This is indicative for the broadness of the pallet of ingredients used in TCM. Three TCM formulas were screened for AA: Ma Huang Decotion (MHD), Antiasthma Simplified Herbal Medicine intervention (ASHMI), and Yu Ping Feng San (YPFS). The TOAT of ASHMI was indicative for an additive effect of herbs used in it. Nevertheless, it can be calculated that one dose of ASHMI is probably too low to cause AA. The TOAT of YPFS was practically zero. This points to a protective interaction of AA. Remarkably, MHD gave a negative TOAT, indicating that the binding to the acetylcholine receptors was increased, which also circumvents AA. In conclusion, our results indicate that TCM is not prone to give AA and support that there is an intricate interaction between the various bioactives in TCM to cure diseases with minimal side effects. 
Redox modulation compounds or flavonoids are potent reactive oxygen species (ROS) scavenger and strengthen the defense system against oxidative stress in the body. Two of the most studied flavonoids, (-)-epicatechin and quercetin, were studied to further unravel their redox modulation effects in the network.

When scavenging ROS, (-)-epicatechin will donate two electrons and become (-)epicatechin quinone, and thus take over part of the oxidative potential of the ROS. In chapter 4 , the chemical reactivity of (-)-epicatechin quinone was determined. When the reactivity would be spread out over the entire molecule, i.e. over the AC-ring and B-ring, this would lead to partial epimerization of (-)-epicatechin quinone to (-)-catechin quinone. In our experiments, (-)-epicatechin quinone was generated with tyrosinase. The formation of (-)-epicatechin quinone was confirmed by trapping with GSH, and identification of (-)epicatechin-GSH adducts. Moreover, (-)-epicatechin quinone could be detected using QTOF/MS despite its short half-life. To detect the epimerization, the ability of ascorbate to reduce the unstable flavonoid quinones into the corresponding stable flavonoids was used. The results showed that the reduction of the formed (-)-epicatechin quinone by ascorbate did not result in the formation of an appreciable amount of (-)-catechin. Therefore it can be concluded that the chemical reactivity of (-)-epicatechin quinone mainly resides in its Bring. This could be corroborated by quantum chemical calculations. Understanding the stabilization of the (-)-epicatechin quinone will help to differentiate between flavonoids and to select the appropriate compound for a specific disorder.

In chapter 5, the redox modulating effects of quercetin (Q) was studied. Q has very strong antioxidant activity because of the combination of two pharmacophores, AC-ring and B-ring. In $\mathrm{Q}$, the $\mathrm{C} 2$ position has the highest occupied molecular orbital because of the interaction of the $3 \mathrm{OH}$ group with 5 and $7 \mathrm{OH}$ groups, as well as the 4 carbonyl group. Therefore, $\mathrm{Q}$ will donate an electron to a free radical from the $\mathrm{C} 2$ positon. The formed quercetin radical ( $\mathrm{Q}^{\circ}$ ) is stabilized by intra molecular scavenging between $\mathrm{C} 2$ and $\mathrm{C}^{\prime}$ ' and the delocalization of the unpaired electron in the B-ring, in which 4'OH group plays an essential role. After intra molecular scavenging, the AC-ring in $\mathrm{Q}^{\bullet}$ is restored to donate another electron to a free radical from the $\mathrm{C} 2$ position. This leads to the formation of the two-electron oxidation product, quercetin quinone methide (QQ). QQ is stabilized through tautomerization. Of the four tautomers of QQ, the tautomer with the carbonyl groups at the maximum distance from each other, is the most abundant one. In line with the hard-softacid-base concept, the soft electrophile QQ preferably reacts with soft nucleophiles such as thiols over hard nucleophiles such as ascorbate and water. QQ can be recycled to Q by ascorbate, and glutathione can adduct to the 6 or 8 position of its AC-ring to form a glutathione adduct. It is speculated that the adduction of thiol group of KEAP1 by QQ will adapt cells against oxidative stress by increasing the synthesis of endogenous antioxidants. However, direct evidence for the adduction of KEAP1 by QQ has not been proved. Our systematic review paves the path to compare the redox modulating activity of $Q$ to that of other polyphenols on a molecular level. This forms the fundament to ultimately select the appropriate redox modulating compound for a specific redox mediated disorder to increase our health. 


\section{Conclusion}

- The energy perspective provides a basis to integrate Eastern and Western medicine

- TCM should be used properly as developed over centuries in China.

- TCM is not prone to give AA and there is an intricate interaction between the various bioactives in TCM to cure diseases with minimal side effects.

- The chemical reactivity of (-)-epicatechin quinone mainly resides in its B-ring.

- The chemical reactivity of QQ is spread over the entire molecule.

- Q will donate an electron to a free radial from the C2 position and form Q* The UE in $\mathrm{Q}^{\bullet}$ stabilized in the B-ring. Then $\mathrm{Q}^{\bullet}$ will donate a second electron to a free radical also from the $\mathrm{C} 2$ position. The $\mathrm{Q}^{\bullet}$ formed is quickly transformed into QQ.

\section{Future perspective}

In the West, we might benefit more from the dynamic interaction between molecules, one of the fundaments of TCM. Using isolated arteries, we could confirm that the accompanying herbs in the Ma Huang Decotion can mitigate the side effects of Ma Huang. Moreover, the dynamic interaction between several herbs in TCM on receptor binding experiments are also - from a Western point of view - unexpected and even contra intuitive. This confirms the dynamic interaction also from the Western point of view and gives new perspective to studying the interaction in TCM.

We still need to extend our knowledge on how antioxidants that differ in hard/softness will have different redox modulation effects. In the redox modulation network, the flavonoid will be oxidized into (-)-flavonoid quinone, which can be regenerated to flavonoid by ascorbate and form GSH adducts by GSH. (-)-Epicatechin is a soft compound, and reacts fast with GSH than ascorbate. However, as relative hard compounds, quercetin and monoHER prefer to react with ascorbate instead of GSH. This difference between redox modulating compounds may behave differently in protecting our body from oxidative damage and inspire to continue furfure unravelling.

Another opportunity is to study other forms of energy, e.g. light. An interesting finding is that TCM "corrects" the light transmitted by the body. This cannot be explained with the Western reductionistic approach, yet. There are numerous other mysterious "forces" in Eastern medicine and other types of traditional medicine that lack a "Western scientific basis", and therefore are left unused and might even be lost. Western scientists should keep on open minded with studying TCM and connect East and West. Undoubtedly, medicine will improve when East and West become more connected, and we get the best of both worlds.

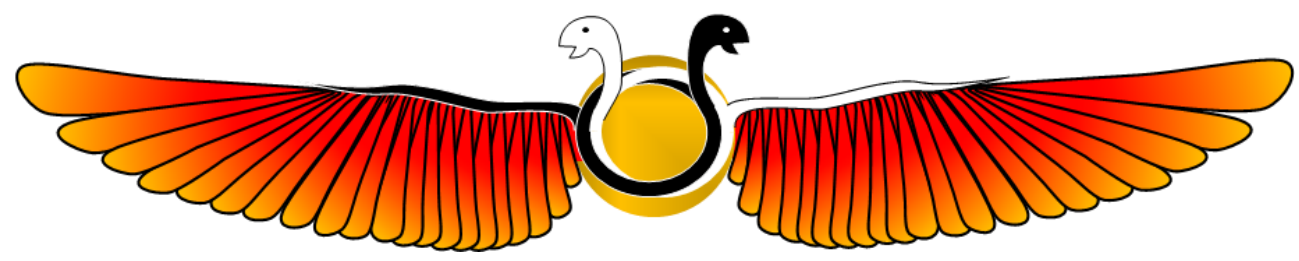






\title{
Chapter 7
}

\section{Nederlandse samenvatting}

\author{
en algemene discussie
}

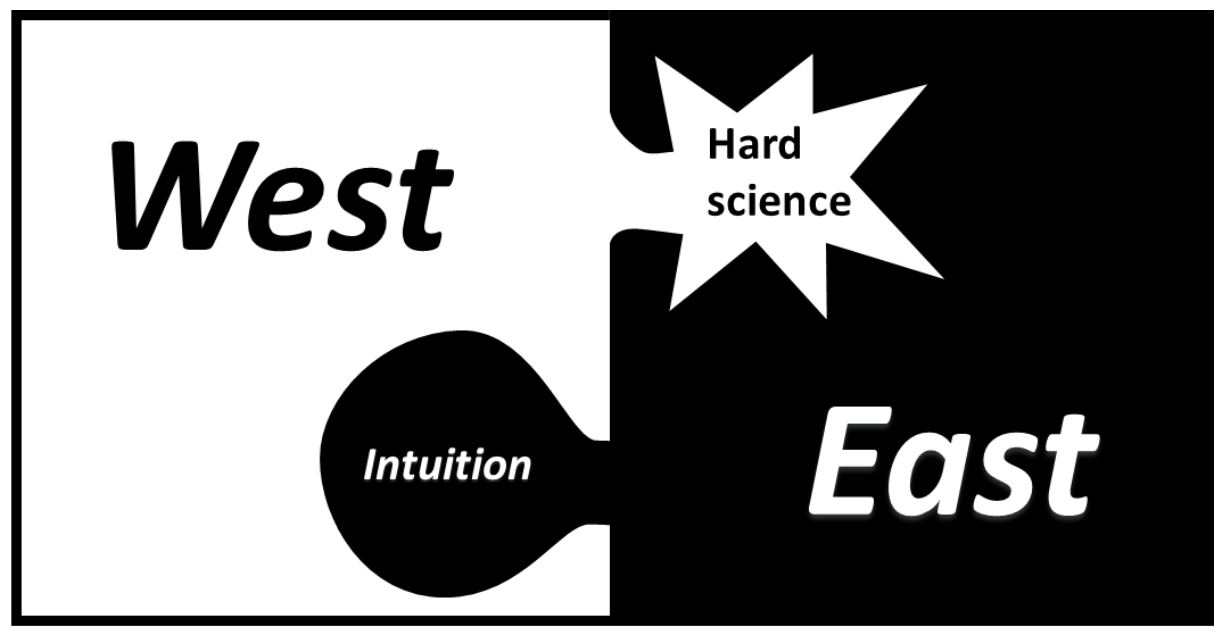


Het centrale thema van het proefschrift is hoe de verbinding te maken tussen westerse en oosterse geneeskunde. Om dit te doen, moeten we naar hun overeenkomsten kijken. Hoofdstuk 1 beschrijft de overeenkomst tussen westerse geneeskunde en oosterse geneeskunde vanuit een energieperspectief. Hoewel de westerse en oosterse geneeskunde zich hebben ontwikkeld en worden toegepast in verschillende culturen, waardoor hun therapeutische concepten anders zijn, zijner opvallende overeenkomsten. In beide werelden leveren tegengestelde krachten de energie die stroomt door netwerken in een organisme, dat het leven voedt. Het vermogen om de balans te handhaven tussen deze tegengestelde krachten, d.w.z. homeostase (West) en harmonie (Oost) geeft "gezondheid" en creëert veerkracht. Bovendien zijn strategieën die worden gebruikt om ziekten te behandelen opvallend gelijk. Redox-modulerende verbindingen en traditionele Chinese geneeskunde (TCM) worden toegevoegd om de verbindingen in de netwerken te vergroten of te versterken om uiteindelijk de stroom van energie aan te passen en de harmonie te herstellen. Het energie perspectief biedt een basis om de oosterse en westerse geneeskunde te integreren en resulteert in de onderzoeken die in andere hoofdstukken van het proefschrift worden beschreven.

In TCM worden kruiden in de juiste verhouding gecombineerd om therapeutische effecten te verhogen en bijwerkingen te verminderen. Wanneer TCM echter in het Westen wordt gebruikt, wordt vaak alleen het kruid met de meest actieve ingrediënt gebruikt. Door verkeerd gebruik heeft TCM waarschijnlijk bijwerkingen. Dit wordt in hoofdstuk 2 geïllustreerd door de cardiovasculaire bijwerkingen van het gebruik van Ma Huang in het Westen. We vonden dat de contractie van placentaire slagaders door Ma Huang zelf veel hoger is dan die van Ma Huang in combinatie met de andere kruiden in Ma Huang Decotion (MHD) zoals die in TCM werd gebruikt. Dit geeft aan dat het samenspel van de verschillende verbindingen in MHD de bijwerkingen van Ma Huang vermindert. Eerdere studies hebben aangetoond dat de andere kruiden in MHD ook de therapeutische werkzaamheid van Ma Huang vergroten. Dit bevestigt dat de cardiovasculaire bijwerkingen gerapporteerd in het Westen het gevolg waren van het oneigenlijk gebruik van Ma Huang, zonder deskundige medische begeleiding. Om het ten volle te kunnen waarderen, moet TCM op de juiste manier worden gebruikt, zoals dat in de loop van de eeuwen in China is ontwikkeld.

In het Westen worden veel bijwerkingen veroorzaakt door een interactie tussen verschillende medicijnen. Eem voorbeeld is de bijwerking die veroorzaakt wordt door het binden van geneesmiddelen aan de acetylcholinereceptoren in de hersenen. Wanneer een aantal geneesmiddelen worden gecombineerd, kan dit substantiële blokkering van deze receptoren veroorzaken, hetgeen bekend staat als anticholinergische stapeling (AA). Het is bekend dat sommige bioactieve stoffen in de TCM de acetylcholine receptoren kunnen blokkeren en dus mogelijk AA veroorzaken. In hoofdstuk 3 werd de AA van TCM gescreend door de verdringing van $\left[{ }^{3} \mathrm{H}\right]$ pirenzepine op acetylcholine receptoren in een ratten hersenen homogenaat te kwantificeren. We hebben een nieuwe eenheid gebruikt om AA uit te drukken, namelijk de "Total Atropine Equivalents" (TOAT). Er werd gevonden dat de TOAT van verschillende kruiden die in TCM worden gebruikt erg divers was, maar relatief laag en zelfs negatief voor sommige kruiden. Dit is illustreert de breedte in activiteit van de ingrediënten die in TCM worden gebruikt. Drie TCM-formules werden gescreend op AA: Ma Huang Decotion (MHD), Antiasthma Simplified Herbal Medicine-Intervention (ASHMI) en Yu Ping Feng San (YPFS). De TOAT van ASHMI wees op een additief effect van de kruiden die erin werden gebruikt. Niettemin kan worden berekend dat een dosis 
ASHMI waarschijnlijk te laag is om AA te veroorzaken. De TOAT van YPFS was vrijwel nul. Dit wijst op een beschermende interactie van AA. Opmerkelijk genoeg gaf MHD een negatieve TOAT, wat aangeeft dat de binding aan de acetylcholine receptoren verhoogd was, wat ook AA omzeilt. Concluderend, onze resultaten geven aan dat TCM niet leidt tot AA en bevestigd dat er een subtiel samenspel is tussen de verschillende bioactieve stoffen in TCM om ziekten te genezen met minimale bijwerkingen.

Redox-modulerende verbindingen zoals flavonoiden reageren realtief snel met reactieve zuurstof species (ROS) en versterken het bescherming tegen oxidatieve stress in het lichaam. Twee van de meest bestudeerde flavonoiden, (-)-epicatechine en quercetine (Q), werden bestudeerd om hun redox-modulatie-effecten in het netwerk verder te ontrafelen.

In de reactie met ROS, doneert (-)-epicatechine twee elektronen en wordt (-)epicatechine quinon gevormd. Hierbij neemt (-)-epicatechine quinon een deel van het oxidatieve potentieel van de ROS over. In hoofdstuk 4 wordt de chemische reactiviteit van (-)-epicatechine quinon bepaald. Wanneer de reactiviteit zou worden gespreid over het gehele molecuul, d.w.z. over de AC-ring en B-ring, zou dit leiden tot gedeeltelijke epimerisatie van (-)-epicatechine quinon tot (-)-catechine quinon. In onze experimenten werd (-)-epicatechine quinon gegenereerd met tyrosinase. De vorming van (-)-epicatechine quinon werd bevestigd door het vangen met glutathione (GSH) en de identificatie van (-)epicatechin-GSH-adducten. Bovendien kon (-)-epicatechine quinon worden gedetecteerd met behulp van Q-TOF / MS ondanks de korte halfwaardetijd. Om de epimerisatie te detecteren, werd ascorbaat gebruikt om het onstabiele flavonoid quinon om te zetten in het overeenkomstige stabiele flavonoid. Het bleek dat de reductie van het gevormde (-)epicatechine quinon door ascorbaat niet resulteerde in de vorming van een aanzienlijke hoeveelheid (-)-catechine. Daarom kan worden geconcludeerd dat de chemische reactiviteit van (-)-epicatechine quinon voornamelijk in de B-ring aanwezig is. Dit werd bevestigd door kwantumchemische berekeningen. Inzicht in de stabilisatie van het (-)-epicatechine quinon zal helpen om onderscheid te maken tussen flavonoiden en om de geschikte verbinding voor een specifieke aandoening te selecteren.

In hoofdstuk 5 werden de redox-modulerende effecten van Q bestudeerd. Q heeft een zeer sterke antioxidantactiviteit vanwege de combinatie van twee farmacoforen, de AC-ring en B-ring. Op de C2-positie in Q is de hoogste bezette moleculaire orbitaal (HOMO) hert meest geconcentreed. Dit kunnen we verklaren door de interactie van de $3 \mathrm{OH}$-groep met 5 en 7OH-groepen, evenals de 4-carbonyl groep. Daarom zal Q een elektron doneren aan een vrije radicaal van de C2-positie. Het gevormde $\mathrm{Q}$ radicaal (Q') wordt gestabiliseerd door intramoleculaire reactie tussen $\mathrm{C} 2$ en $\mathrm{C} 1$ ' en de delocalisatie van het ongepaarde elektron in de B-ring, waarbij de 4'OH-groep een essentiële rol speelt. Hierbij wordt de AC-ring in Q' "hersteld" om een ander elektron te doneren aan een vrije radicaal van de C2-positie. Dit leidt tot de vorming van het twee elektron oxidatieproduct, Q quinon methide (QQ). QQ wordt gestabiliseerd door tautomerisatie. Van de vier tautomeren van QQ is de tautomeer waarbij de carbonyl groepen op de maximale afstand van elkaar zitten, de meest voorkomende. In overeenstemming met het hard-zachte zuur-base concept reageert de zachte elektrofiel QQ sneller met zachte nucleofielen zoals thiolen dan met harde nucleofielen zoals ascorbaat en water. In de reactie met ascorbaat wordt QQ gerecycled naar Q. GSH addeert QQ op de 6 of 8 positie waarbij een GSH-adduct wordt gevormd. Gespeculeerd is dat de adductie door QQ van thiol-groep van Kelch-like ECH-associated protein 1 (KEAP1), een cel zich aanpast tegen oxidatieve stress door de synthese van endogene antioxidanten te verhogen. Direct bewijs voor de adductie van KEAP1 door QQ 
is echter niet gevonden. Onze systematische review effent het pad om de redoxmodulerende activiteit van $\mathrm{Q}$ te vergelijken met die van andere polyfenolen op moleculair niveau. Dit vormt het fundament om uiteindelijk de juiste redox-modulerende verbinding te selecteren voor een specifieke redox-gemedieerde aandoening.

\section{Conclusie}

- Het energieperspectief biedt een basis om Oosterse en Westerse geneeskunde te integreren

- TCM moet correct worden gebruikt, zoals in de loop van de eeuwen in China is ontwikkeld.

- TCM is niet vatbaar voor AA en er is een subtiel samenspel tussen de verschillende bioactieve stoffen in TCM om ziekten te genezen met minimale bijwerkingen.

- De chemische reactiviteit van (-)-epicatechine quinon zit voornamelijk in de B-ring.

- De chemische reactiviteit van QQ is verspreid over het gehele molecuul.

- Q doneert een elektron aan een vrije radiaal vanuit de C2-positie en vormt Q* Het ongepaard elektron in $\mathrm{Q}^{*}$ gestabiliseerd in de B-ring. $\mathrm{Q}^{*}$ doneert een tweede elektron aan een vrije radicaal, ook vanuit de C2-positie. Het gevormde $\mathrm{Q}^{\bullet}$ wordt snel omgezet in QQ.

\section{Toekomst perspectief}

In het Westen kunnen we meer profiteren van de dynamische interactie tussen moleculen, een van de fundamenten van TCM. Met behulp van geïsoleerde slagaders kunnen we bevestigen dat de begeleidende kruiden in de MHD de bijwerkingen van Ma Huang kunnen verlichten. Bovendien is de dynamische interactie tussen verschillende kruiden in TCM op receptor binding experimenten ook - vanuit een Westers oogpunt onverwacht en zelfs contra intuïtief. Dit bevestigt de dynamische interactie ook vanuit het westerse gezichtspunt en geeft een nieuw perspectief voor het bestuderen van de interactie in TCM.

We moeten nog steeds onze kennis uitbreiden over hoe antioxidanten die verschillen in hard/zachtheid verschillende redox-modulatie-effecten zullen hebben. In het redoxmodulatienetwerk zal de flavonoid worden geoxideerd tot enn flavonoid quinon, dat door ascorbaat geregenereerd kan worden tot het oorspronkelijke flavonoid, en GSH-adducten met GSH kan vormen. (-)-Epicatechine is een relatief zachte verbinding en reageert sneller met GSH dan met ascorbaat. Als relatieve harde verbindingen geven Q en monoHER echter de voorkeur om te reageren met ascorbaat in plaats van GSH. Door dergelijke verschillen zullen redox-modulerende verbindingen zich anders gedragen bij het beschermen van ons lichaam tegen oxidatieve schade. Hopelijk inspireert ons onderzoek om verder te gaan met ontrafelen van de werking van redox modulerende stoffen.

Een andere mogelijkheid tot vervolgonderzoek is om andere vormen van energie te bestuderen, bijvoorbeeld licht. Een interessante bevinding is dat TCM het door het lichaam uitgezonden licht "corrigeert". Dit kan nog niet worden verklaard met de westerse reductionistische benadering. Er zijn talloze andere mysterieuze "krachten" in de oosterse geneeskunde en andere vormen van traditionele geneeskunde die een "westerse wetenschappelijke basis" missen en daarom ongebruikt blijven en zelfs verloren kunnen gaan. Westerse wetenschappers moeten "open minded" blijven door TCM te bestuderen en 
te proberen Oost en West met elkaar te verbinden. Ongetwijfeld zal de geneeskunde verbeteren wanneer Oost en West meer verbonden raken en we het beste uit beide werelden halen.
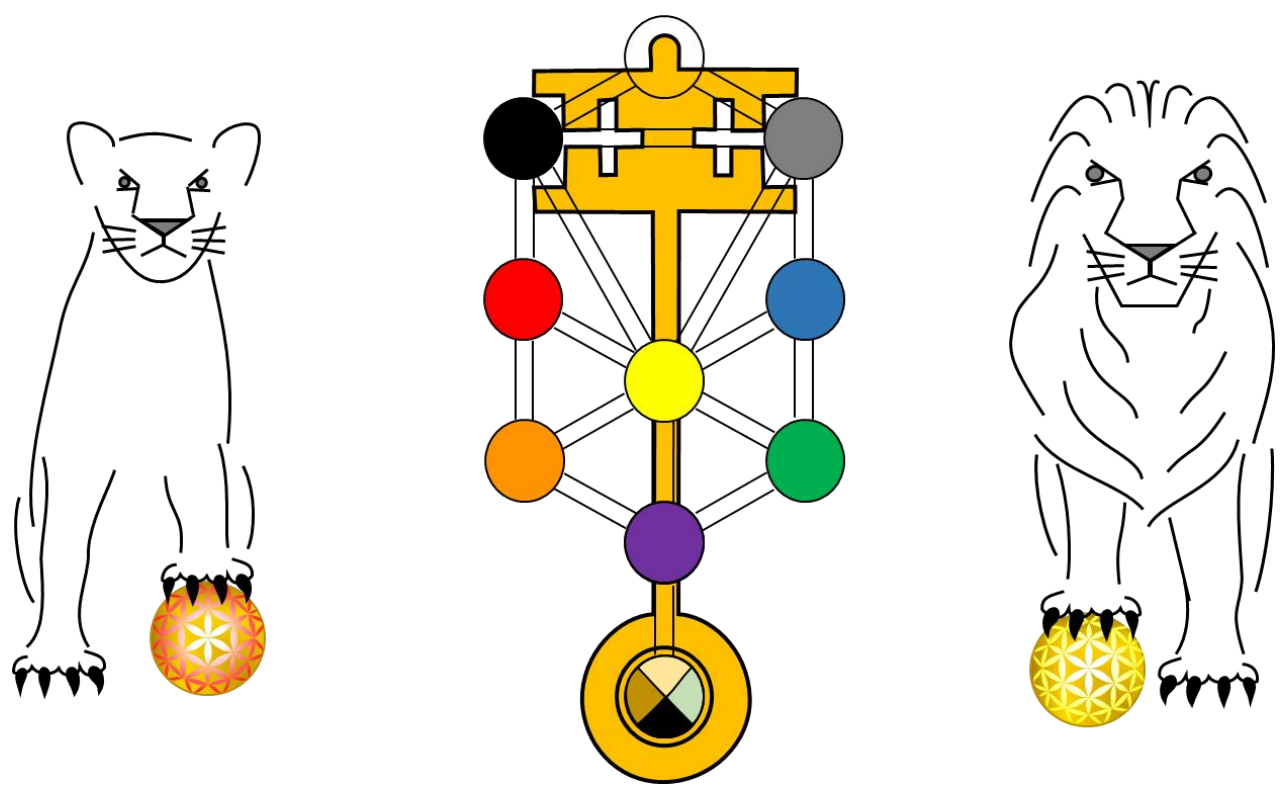



\section{Valorization addendum}




\section{Relevance}

In this thesis, an attempt is made to connect Western medicine and Eastern medicine from an energy perspective. Namely, there are opposing forces in both worlds that create energy that flows in a network to fuel life. The relevance and impact of our research to the society is potentially of great influence. The introduction of TCM in the Western markets will bring a lot of business and might improve the Quality of Life for patients.

\section{Target groups}

In our research we used a Western pharmacological point of view to try to unravel the mechanism of TCM to make it more acceptable. The similarities in the basic fundaments between Western and Eastern medicine can be used to better connects both scientifically as well as from a regulatory point of view, so West and East can benefit from each other. Patients and doctors in the West might start thinking about using TCM when the Western medicine fails to be effective or results in too much side effects.

\section{Activities and products}

TCM has developed in China over centuries and is used still according to the traditional way. However, in the West some serious side effects have been ascribed to the use of TCM. Our research indicates that this is due to the improper use of TCM and stresses that TCM should be used in the right way, preferably under the supervision of appropriate trained medical professionals.

The redox modulating activity of two key flavonoids was further unraveled on a molecular level in the thesis. A systematic unravelling of more redox modulating bioactives on molecular level can give the fundament for selecting the appropriate redox modulating compound to protect against a specific redox mediated disorder.

\section{Innovation}

The dynamic interaction between several herbs in TCM on receptor binding experiments are also - from a Western point of view - unexpected and even contra intuitive. This confirms the dynamic interaction also from the Western point of view and gives new perspective to studying the interaction in TCM.

Understanding the redox modulation of bioactives and using it to predict their impact on health.

Patients and doctors can ultimately decide which type of treatment they prefer for a specific condition. They realize that both have strengths and weaknesses, and are able to select the type that suits a specific patient, situations and illnesses the best.

\section{Planning and realization}

Before Western and Eastern medicine are really connected, more research needs to be performed. The energy perspective might form a good basis. The Jun-Chen-Zuo-Shi and hard-soft-acid-base concept can be used to study the dynamic interaction of compounds to correct an imbalanced energy. We definitely need to know more about how compounds behave in these concepts. Moreover, there might other ways to connect Western and 
Eastern medicine. This thesis provides a new lead that hopefully inspires to keep an open mind in studying medicines of different cultures. 


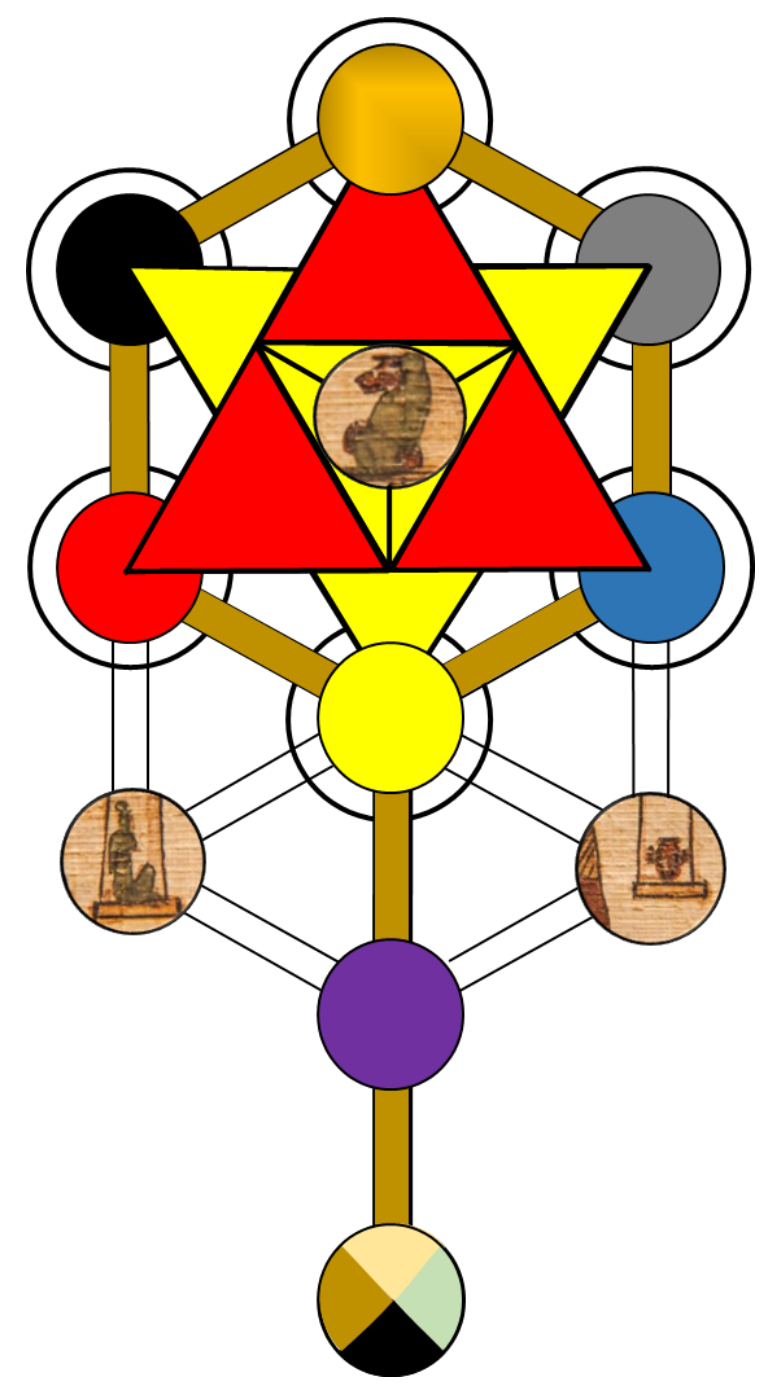




\section{Chinese translation}




\section{前言}

医疗人员在各个社会中具有很高的地位, 这反映了大多数人的首要愿望是具有良 好的健康。这些医疗人员用特定的且容易理解的符号表达自己。

除了这些相似的地方，药物在不同的文化中有着不同的发展。比如，西药跟东方 药物十分的不同。他们建立在不同的基础之上, 有各自的优点和局限。但是, 两种 类型的药物是可以互补的, 我们可以通过连接西药和东方药物来获益, 两全其美。 这符合 2008 年第 61 届世界卫生组织大会通过的“全球公共卫生, 创新和知识产权战 略和行动计划”, 其中指出应通过刺激研究和创新进一步发展传统医学。这促使我们 进一步揭示中药的作用方式和毒性的分子机制, 将中药与西药连接起来。

\section{西药的基础}

西药以“证据为基础”, 从分子水平到对患者的最终治疗效果都是理性的。它从草 药的使用开始, 变化到分离和使用草药中活性最强的成分, 然后到使用 (半) 合成 化合物。最近获得的关于药物化学结构和特殊受体的知识, 可以阐明药物的分子作 用模式, 并彻底改革了西药。作为西药的主要成就之一, 抗生素拯救了数百万人生 命。药物开发的目标是增加其活性和特异性, 并设计具有强烈, 特异和即时效果的 药物。药物的作用在非常严格的指导方案下进行临床实验测试, 最终获得基于证据 的标准化疾病诊断和治疗指南。

\section{东方药物的基础}

东方药物则有主观的基础。中药已经在中国发展了几个世纪, 并且仍然按照传统 方法使用。东方药物是中药兴起的动力之一。在中药中, 各种草药以特定比例相组 合, 以获得最佳的效果。这其中并没有涉及具体的受体。一般来说, 中药的效果相 对柔和, 治愈疾病需要很长时间。但是, 中药的副作用很小。中医根据传统和经验 对患者进行“个性化”方式治疗。

\section{西药的局限性}

从西方的角度来看, 毫无疑问, “科学的”西方方法远比东方的“主观”方法更优越。 然而, 无数的例子表明, 在西方, 谦虚的态度更适合。其中之一是无法解释其效果 的扑热息痛。虽然扑热息痛是最常用的药物并且已经使用了几十年, 但其分子作用 模式尚未阐明。这表明神秘的“安慰剂”作用有助于扑热息痛的治疗效果。另一方面, 许多西药从长远来看不会有效。与中医相比, 西药的半衰期非常短。甚至抗生素也 会失去其药性, 因为微生物可以变得耐药而不对该药物起作用。此外, 西药的作用 通常不像宣布的那样具有特异性, 并且它们不会作用于单一受体。例如, 许多药物 显示出抗胆碱能活性的副作用。当这些药物混合使用给予患者时, 这可能会引起“抗 胆碱能累积”。

此外, 批判性检查表明, 许多对照临床试验实际上可能会给出错误的结果。人们 越来越意识到, 即使看起来绝对正确的科学结果也可能是不正确的。此外, 大多数 制药厂的目标是尽可能多地赚钱, 而不是为患者谋福利。在西药中, 欺计和操纵科 
学家和医生并不少见。制药业甚至被指责发明和传播疾病, 其唯一目标是推销其“神 奇疗法”（例如利他林）。

\section{东方药物的局限性}

中药的分子作用机理尚不清晰。例如, 经络并没有科学的证据。这意味着中药的 基础在西方被否定, 东西方没有得到连接。然而, 在西方也经常发现中药对于治疗 疾病是有价值的，特别是当合成设计药物没有效果时。但其中一个问题是中药的使 用导致了副作用。例如，麻黄是一种在中国几个世纪以来用来治疗哮喘的草药，通 过将其与其他草药结合, 并且使用相对较低的剂量, 由麻黄所引起的副作用降低至 相对安全的水平。然而, 在美国, 麻黄在没有与其他草药的组合使用的情况下, 且 以相对高的剂量使用, 用来减肥或作为兴奋剂。麻黄的这种不当使用引起了严重的 心血管问题。这虽然符合旨在实现快速强大效果西药的战略, 但却导致了不恰当中 药使用, 并导致副作用。这给中药带来了不好的声誉, 并妨碍了正确欣赏中药。为 了给中药一个“科学”基础并阐明其分子机制, 西方科学需要以正确使用中药的方式 研究中药。此外, 西方科学家应该对东方药物持开放态度, 并接受西方可以向东方 学习。

\section{如何使中西药相结合}

连接西方和东方药物, 我们不应该专注于差异, 而应该寻找相似之处。在第一章 中, 我们讨论了西药和东方药物从能量角度来看的惊人相似性。二者均含有对立力 量, 对立力量提供了流经网络的能量, 从而为生命提供动力。在这个概念中, 健康 是指生物体维持这些对立力量之间平衡的能力, 即动态平衡（西方）和和谐（东 方）, 这会增加生物体的弹性。此外，二者用于治疗疾病的策略非常相似。添加氧 化还原调节化合物和中药以增加或加强网络中的连接, 以最终调节能量流并恢复其 平衡。

因此, 能量观点为连接东西方药物提供了基础。它告诉我们应该研究什么。例如, 我们需要扩充各种氧化还原调节剂如何在网络中产生不同影响的知识。此外, 在西 方, 我们可以从分子之间的动态相互作用（中药机理）中受益更多。另一个机会是 研究其他能量形式, 例如光。一个有趣的发现是中药可以“纠正”身体发出的光, 但 是这还不能用西药来解释原因。东方药物和其他类型的传统药物中还有许多其他神 秘的“力量”缺乏“西方科学基础”, 因此被遗忘或丢失。毫无疑问, 当东西方药物连接 更加紧密时, 医学将得到改善, 人类会最终受益。

\section{论文研究目的和框架}

为了连接西药和东方药物, 我们从西方的角度探索东方药物。在第一章中, 从能 量的角度建立了东西方药物之间联系的桥梁。在第二章中, 探讨了麻黄在中药中如 何防止了在西方使用中观察到的副作用。第三章中, 探究了中药的抗胆碱能积累作 用, 因为中药中含有许多与毒草碱受体结合的化合物。在第四章和第五章中, 研究 了表儿茶素和槲皮素的氧化还原调节机制。 


\section{第一章 从能量的角度连接东西方药物}

\section{摘要}

虽然西药和东方药物时分开的，但是当我们从能量的角度来看这些医学的基本原 则时, 它们有着惊人的重叠之处。在这两个世界中, 对立的力量提供了通过生物体 网络中流动的能量, 进而为生命提供动力。在这个概念中, 健康是指生物体维持这 些对立力量之间平衡的能力, 即动态平衡 (西方) 和和谐（东方）, 这会产生弹性。 此外, 用于治疗疾病的策略非常相似, 即通过改变网络中的连接来调整能量流。能 量的观点为结合东西方药物奠定了基础, 开辟了研究的新方向, 以实现二者共赢。

关键词: 西药, 东方药物, 对立力量, 氧化还原, 阴阳, 弹性

\section{1. 引言}

很多人希望他们的生命结束后可以奔赴天堂而免于地狱的灼烧, 可以他们也许不 知道，他们不用担心在地狱中燃烧，他们地球上时已经开始燃烧了。这种燃烧是一 种不同寻常的东西引起的, 即氧气。大多数人认为我们需要氧气, 氧气对我们的色 号干农活至关重要, 但是, 氧气的活性很高, 因此会有毒性。她会使我们像蜡烛一 样慢慢燃烧。比如老年斑, 老年斑就是燃烧的蛋白质, 我们的身体无法清除它, 在 体内不断地积累，因此变得有毒。又比如 DNA 的燃烧可以致癌。

\section{2. 对立力量}

氧气引起的燃烧称为“氧化”。幸运的是，还有一种反作用力，即“还原”。还原和 氧化的第一个字母组合起来即“氧化还原”。Redox 描述了氧化力和还原力之间的平 衡（图 1)。正如引言中所述, 氧化还原的能量是生命的驱动力。然而, 氧化还原能 量也会造成损害，好与坏并存。

任何事物都包含好与坏也在 15 世纪被西方药理学家 Paracelsus 指出, 他说: “获 得一件非常好的东西, 首先必须与的东西分开。不付出好的价格无法获得好的艺术。 为了得到你想要的东西, 你也必须面对你不想要的东西 “。悖论之中存在另一个悖论。 比如, DNA 的氧化损伤偶然会产生一种更适应其环境的突变生物。这是“适者生存” 进化论的基础。因此，“损害”对于生存，恢复和进步也是必不可少的。

东方比西方更早的意识到一切事物都包含对立的力量, 这也是太极符号的最佳象 征, 阴阳平衡, 在黑色圆圈的部分, 有一个小的白色圆圈, 这可视化符号中的双重 悖论之一。实际上，太极符号是人类制作的第一个分形符号之一。因此，比起西方 的氧化学符号, 使用太极符号可以更好地体现氧气的矛盾性（如图 2）。

阴阳是控制生命和宇宙的对立力量。阴主要影响身体, 物质方面; 阳主要影响心 理, 精神方面。与还原和氧化类似, 它们相互作用并相互平衡, 阴上阳下, 阴下阳 上。对立力量形成了在我们体内循环流动的能量或气。身体器官通过经络或通络的 渠道调节和保护气。当气不和谐时会发生疾病。治疗方法主要是纠正阴阳平衡, 从 而调整气，使其恢复和谐。 


\section{3. 西方健康概念}

在我们的生命中, 氧化还原的能量发生变化（图 3）。在健康的身体中, 氧化还 原的能量在非常严格的限度内保持很高的水平, 我们称之为动态平衡, 与阴阳平衡 相似。健康可以定义为适应挑战的能力。当我们遇到挑战时, 会对能量产生攻击, 能量下降, 但我们的身体有弹性吸收挑战并反弹。在反弹中, 甚至有一个暂时的改 善, 弹性增加。因此, 一点坏对身体有好处, 这符合上面描述的双重悖论。当挑战 增加并且持久时, 我们会遇到问题。例如, 由于慢性疾病, 能量降低并在较低能量 水平达到新的平衡。在这种情况下, 我们甚至无法应对一个小的挑战。所有能量可 能会流失，生命也会结束。

我们身体的这种弹性来自哪里? 在西方科学中, 身体由分子组成, 所有分子紧密 相连。这些连接可以通过系统生物学中的网络图来显示（图 4）。在通常由酶催化并 由各其他种分子控制的过程中, 一种分子可以转化为另一种分子。这些反应的能量 来自氧化还原。这些反应组合在一起形成一个弹性安全网, 使得身体可以轻松应对 挑战, 甚至可以反弹并增加弹性。在生病的机体中, 许多反应丢失, 安全网变得脆 弱。弹性降低。连接的重要性可以一个比喻轻松记住。“Wellness”的前两个字母给出 “We”。当“我们”在一起时, “我们”是相互联系和健康的。在每个社会和文化中都是 如此。“Illness”的第一个字母给出“I”。当“我”独自一人时, “我”被孤立, 并且不健康。

\section{4. 东方健康概念}

连接在东方的弹性和健康概念中也非常重要。在中国哲学中，所有生物都由五个 元素组成: 木, 火, 土, 金和水（图 5)。这五个元素相互作用并相互补偿以找到适 当的平衡。五种元素之间相生相克。元素之间的相互作用受阴阳平衡的支配。每个 生物体都在不断努力实现这五种元素的最佳动态平衡或协调, 维持健康。在这种情 况下, 身体的所有重要器官都可以应对挑战, 从而提供弹性。该网络中的元素之间 的不平衡将带来疾病。

\section{5. 连接东西方}

尽管东西方药物之间存在很大差异，但显然上述表明它们所使用的基本原理非常 相似。两者都利用相反的力量来产生能量, 这些能量通过网络流动以提高生命力。 两种类型的药物都利用这一概念来治疗疾病或增加健康。为了增加健康, 两者都旨 在加强连接或使新的连接重新调整平衡, 即稳态（西方）或和谐（东方），并加强 网络（弹性）。

\section{1. 西药}

在西方科学中, 当分子相互作用时就会产生联系, 这可以提供保护机能。一个重 要的问题是为什么一些连接可以建立而其他的连接不可以。为什么一些分子之间发 生反应而其他分子之间不同，以及能量是如何流经网络的。这可以根据 Pearson 的 hard-soft-acid-base 概念来解释。在 hard-soft-acid-base 概念中, “hard”适用于相对较小, 具有高电荷且弱极化的物质, “soft”适用于相对较大, 具有低电荷或无电荷, 强极化 的物质。因此, “hard”酸（亲电试剂）和“hard”碱（亲核试剂）具有大的轨道重叠, 
彼此快速反应。此外, 由于大的轨道重叠, “soft”亲电子试剂与“soft”亲核试剂反应迅 速。hard-soft-acid-base 概念也可以推广到其他类型的亲电试剂和亲核试剂, 不一定 是酸或碱。

当能量集中在分子的一个位置时，像放大镜的焦点，该分子变得“hard”并且非常 活泼。例如, 在羟基中, 未配对电子的所有能量都位于单个原子（即氧）上。因此 它是我们体内反应最活跃的分子（图 6）。羟基自由基可与它遇到的任何分子快速反 应。这将导致不受控制的能量流动并对我们的身体造成伤害。䚞皮素和 monoHER 等 抗氧化剂是 hard 电子供体, 可与羟基和其他活性氧物质（ROS）等 hard 电子受体快 速反应。通过接受电子, ROS 被转化为活性较弱的化合物, 不会破坏细胞。例如, 通过接受电子, 羟基被转化为羟基阴离子, 在质子化后, 羟基阴离子变成水。通过 提供电子, 槲皮素和 monoHER 分别转化为相应的 soft 氧化产物, 葪皮素醌和 monoHER 醌（图 6）。这些氧化产物充当“soft”亲电试剂并选择性地与“soft”亲核试 剂如硫醇反应而不是“hard”亲核试剂如水。如下一段所述, 我们可以使用这种 hardsoft-acid-base 概念来解释挑战的生物效应和抗氧化剂的调节作用。

对氧化还原能量的挑战通常来自 ROS 的 hard 能量。 ROS 的 hard 能量会破坏细胞。 这是因为 hard 能量可以与细胞中的所有物质反应, 包括蛋白质, 脂质和 DNA, 最终 会破坏细胞。细胞中有一个传感器可以被 hard 能量打开。通过打开传感器, 细胞反 应并产生更多的抗氧化剂。该传感器是 KEAP1。ROS 可以氧化 KEAP1 上的硫醇基 团, 氧化的 KEAP1 将从与 Nrf2 复合物中解离。释放的 Nrf2 将被传至细胞核并刺激 许多解毒和抗氧化基因的转录（图 7A）。一个小的挑战最终将加强细胞的防御系统 并增强细胞的弹性。

当我们添加一种抗氧化剂如 monoHER 时, ROS 的 hard 能量将被 monoHER 吸收 和软化, 并形成 soft 的 monoHER 醌（图 7B）。这种 monoHER 醌的 soft 能量不会破 坏细胞中 DNA 和其他重要的分子, 但它可以很好地打开传感器。这是因为传感器 上的开关是 soft 亲核试剂, 即硫醇或颈基 ( $\mathrm{SH}$ ) 基团。与硬 hard-soft-acid-base 概念 一致, soft-醌将与 KEAP1 上的 soft 硫醇基团有效且选择性地反应。将能量由 hard 转 化为 soft, 可以解释抗氧化剂的直接保护作用以及对弹性的有效增加作用。因此, 抗 氧化剂不仅可以保护挑战的威胁; 它也会因挑战而增加反弹, 提供更大的弹性。

\section{2. 中药}

在东方哲学中, 当阴阳之间存在不平衡时, 能量的流动是不正确的, 丧失和谐, 产生疾病。能量的不平衡可以通过针炎或经络穴位或中药来纠正。这些治疗旨在重 新调整阴阳的能量, 重新获得五行的和谐。

中药主要是各种草药的结合。他们的组合基于“君臣佐使”, 被称为四个负责任的 角色（图 8)。每种草药都具有特定的功能, 它们的相互作用使治疗更加强大并具有 特异性。“君” (君主) 草药是主要的活性药, 具有治疗疾病的主要作用, 类似于 hard 分子。“臣”药（臣僚）增强了君草的疗效。“佐”药（僚佐）调节君药和臣药的作 用, “使”药 (使者) 协调其他药的作用, 类似于氧化剂如何使 hard 能量转换为 soft 能量。总之, 通过草药的配伍, 主要治疗效果增强, 并且副作用减少。

例如, 用于治疗例哮喘的麻黄汤 (图 9) 药方中, 麻黄是最有效的草药, 君药, 它用于促进出汗, 并使肺部扩张。桂芝作为臣药辅助麻黄, 促进发热减少头痛和喘 
气, 促进发汗, 减少麻黄的副作用。杏仁和甘草分别作为佐药和使药, 共同提高马 黄和桂枝的治疗效果。杏仁是一种苦涩温暖的佐药, 可增强肺功能, 增强麻黄的出 汗效果。甘草, 具有甜美和温暖性质的施药, 协调其他草药的作用, 使组合的治疗 效果更具体。通过梳理草药, 增强了马黄的治疗效果, 并且麻黄引起的副作用也降 低到相对安全的水平。中药之间错综复杂的相互作用可以协调体内的热量和能量, 从而达到良好的健康状态。

\section{6. 结论}

西方和东方的健康哲学似乎是世界分开的。在西方, 有机体被简化为一系列分子。 这种方法在生成有效药物方面非常成功。然而, 西医的局限性正变得越来越明显。 显然，在西方还原论的方法中，有些东西丢失了。这可能是“情感”和“感觉”最好的证 明 - 虽然生命中必不可少 - 但不能仅通过观察分子来解释。东方医学使用更直观的方 法。虽然从西方的角度来看, 东方医学不是“科学的”, 但人们已经从这种直观的医 学中获益了数千年, 而东方和其他传统类型的医学仍然被大多数人使用。尽管存在 差异，但当我们从能量的角度来看西方和东方医学时，这两个世界似乎都基于类似 的原理（图 9）。普遍原则是，对立的力量产生的能量流经生物体中的网络，从而为 生命提供动力。这种观点可他能为整合东方医学和西方医学奠定基础, 并为研究开 辟新的方向，填补两个世界之间的知识空白。

\section{7. 展望}

能源观点为连接东西方药物创造了一座桥梁。在西方, 我们可能从分子之间的动 态相互作用中获益更多，这是中医的基础之一。使用分离的血管动脉，我们可以确 认麻黄汤中草药可以减轻麻黄的副作用。此外, 中药中几种草药对毒葍碱受体结合 的动态相互作用也是从西方的观点来看 - 意想不到甚至反直觉。能量观点还表明, 我 们仍然需要扩展我们对硬度/柔软度不同的抗氧化剂如何具有不同氧化还原调节效应 的知识。另一个机会是研究其他形式的能量, 例如光。一个有趣的发现是 TCM “纠 正”身体传播的光。这还不能用西方简化方法来解释。东方医学和其他类型的传统医 学中还有许多其他神秘的“力量”缺乏“西方科学基础”, 因此未被使用甚至可能丢失。 毫无疑问, 当东西方联系更加紧密时, 医学将会得到改善, 我们将获得两全其美的 优势。 


\section{讨论和总结}

本论文的核心主题是如何将东西方药物连接起来。为了做到这一点, 我们应该从 它们的相似之处开始研究。第 1 章从能量的角度阐述了西药与东方药物的相似之处。 虽然西药和东方药物发展并应用于不同的文化, 其治疗概念不同, 但是我们却发现 它们之间有惊人的相似之处。在这两个世界中, 对立的力量提供了通过生物体网络 中流动的能量, 从而为生命提供动力。在这一概念中, 健康是指生物体维持这些对 立力量之间平衡的能力, 即动态平衡 (西方) 和和谐 (东方), 这会增加生物体的 弹性。此外, 二者用于治疗疾病的策略非常相似。添加氧化还原调节化合物和中药 以增加或加强网络中的连接, 最终调节能量流并恢复其平衡。能量观念的引入提供 了结合东西方药物的基础, 也为了论文其他章节所述的研究成果奠定了基础。

在中医中，草药以适当的比例组合以增加治疗效果并减少副作用。然而，当中药 在西方使用时, 通常仅使用草药中最有活性成分。这种不正确的使用可能会产生副 作用。在第 2 章中, 我们举例说明了麻黄在西方观察到的心血管副作用。我们发现, 麻黄可以使得胎盘血管收缩, 而且作用远高于麻黄与其他药物一起使用的麻黄汤。 这表明麻黄汤中各种化合物的相互作用减轻了麻黄的副作用。以往的研究表明, 麻 黄汤中的其他草药也增加了麻黄的治疗效果。这证实了西方报告的心血管副作用是 由于麻黄缺乏专业人士的监督, 以至于使用不当造成的。为了充分发挥其益处, 中 药应该按照在中国发展几个世纪的方法使用。

在西方, 药物的组合也可能会引起严重的副作用。例如, 许多西药对大脑中的乙 酰胆碱受体表现出一定的亲和力。当这些药物与受体结合时, 这可能使这些受体阻 断, 称为抗胆碱能累积 ( AA)。中药中的一些生物活性物质可以阻断乙酰胆碱受体, 因此引起 $\mathrm{AA}$ 。在第 3 章中, 通过用量化放射性 $\left[{ }^{3} \mathrm{H}\right]$ 哌仑西平与兔子脑部匀浆中的乙 酰胆碱受体的置换, 篮选了中药的 AA。我们采用了一个新的单位来表达 AA, 即总 阿托品等价物（TOAT）。结果发现, 不同草药的 TOAT 差别很大, 但总体相对较低, 甚至某些草药的 TOAT 为负值。这表明中药中使用十分多。同事, 篮选了三种中药 药方的 AA：麻黄汤, 阿吸美和玉屏风散。阿吸美的 TOAT 表明草药的抗胆碱能作用 具有累加效应。然而, 计算表明阿吸美的剂量太低而不能引起 AA。玉屏风散的 TOAT 几乎为零。这表明它可以减少 AA。值得注意的是, 麻黄汤的 TOAT 为负值, 表明它增加了与乙酰胆碱受体的结合加, 从而不显示 $\mathrm{AA}$ 。结果表明, 中药不易产生 $\mathrm{AA}$, 并表明中药中的活性物质之间存在复杂的相互作用, 以治疗疾病, 并减少副作 用。

氧化还原调节化合物或黄酮类化合物是有效的活性氧物质清除剂, 并且加强机体 防御系统以抵抗体内的氧化应激。我们选取了两种研究最多的黄酮类化合物表儿茶 素和槲皮素, 以进一步揭示它们的氧化还原调节作用。

当清除活性氧物质时, 表儿茶素将捐献两个电子并变成表儿茶素醌, 从而接收活 性氧物质的部分氧化能力。在第 4 章中, 我们检测了表儿茶素醌的化学反应力。当 反应力分山到整个分子上时, 即在 $\mathrm{AC}$ 环和 $\mathrm{B}$ 环上, 会导致表儿茶素醌部分差向异 构化为儿茶素醌。在我们的实验中, 用酪氨酸酶产生表儿茶素醌。通过鉴定与谷胱 甘肽反应生成的表儿茶素-GSH 结合物来证实表儿茶素醌的存在。此外, 可以使用 QTOF/MS 检测表儿茶素醌, 尽管其半衰期较短。为了检测差向异构化, 使用抗坏血酸 将不稳定的类黄酮醌还原成相应的稳定的类黄酮。结果表明, 抗坏血酸还原形成的 
表儿茶素醌中没有监测到儿茶素的形成。因此可以得出结论, 表儿茶素醌的化学反 应性主要在于 $\mathrm{B}$ 环, 并可以通过量子化学计算得到证实。了解表儿茶素醌的稳定性 将有助于区分黄酮类化合物, 并为特定疾病选择合适的化合物。

在第 5 章中, 我们研究了檞皮素的氧化还原调节作用。 $\mathrm{AC}$ 环和 $\mathrm{B}$ 环的组合, 使 得䚞皮素具有非常强的抗氧化活性。在槲皮素中, 由于 3-羟基与 5 和 7-羟基以及 4羰基的相互作用, C2 位置具有最高的分子占据轨道。因此，檞皮素将从 C2 位置向 自由基贡献一个电子。形成的槲皮素自由基通过电子 $\mathrm{C} 2$ 和 $\mathrm{C} 1$ '之间的分子内清除和 $\mathrm{B}$ 环中未配对电子的离域得到稳定, 其中 4'-羟基对这一过程起重要作用。在分子内 清除后, 槲皮素自由基中的 $\mathrm{AC}$ 环得到恢复, 可以又从 $\mathrm{C} 2$ 位置向另一个自由基提供 另一个电子。这形成了双电子氧化产物, 檞皮素醌。槲皮素醌通过互变异构化得到 稳定。在檞皮素醌的四种互变异构体中, 羰基具有最大间距的互变异构体是最多的 互变异构体。依据 hard-soft-acid-base 概念, soft 亲电试剂槲皮素醌会优选与 soft 亲核 试剂反应, 如硫醇反应, 而不是 hard 亲核试剂反应, 如抗坏血酸和水。槲皮素醌可 以通过抗坏血酸再循环到檞皮素; 谷胱甘肽可以加合到檞皮素醌的 $\mathrm{AC}$ 环的 6 或 8 位 以形成谷胱甘肽加合物。据推测, 槲皮素醌对 KEAP1 統基的加合将增加内源性抗氧 化剂的合成从而增加细胞抵抗氧化应激能力。然而, 槲皮素醌与 KEAP1 结合物的直 接证据尚未可知。本研究为在分子水平上比较檞皮素和其他多酚物质氧化还原调节 能力奠定了基础。最终为特定的氧化还原失调的疾病选择合适的氧化还原调节化合 物奠定基础, 提高了我们的健康。

\section{结论}

- 能量观点为整合东西方药物提供了基础

- 中医应该按照在在中国开发几个世纪以来的方式使用。

- 中药不易产生 AA, 并且中医中的各种生物活性物质之间存在复杂的相互作 用, 以治疗疾病, 降低副作用。

- 表儿茶素醌的化学反应能力主要存在于其 B 环。

- 䚞皮素醌的化学反应能力分布在整个分子。

- 槲皮素将从 C2 位置向自由径向提供电子并形成檞皮素自由基。檞皮素自由 基的未配对电子稳定在 $\mathrm{B}$ 环中。那么槲皮素自由基也会从 C2 位置向自由基 提供第二个电子，葪皮素自由基很快转变为葪皮素醌。

\section{展望}

在西方, 我们可从中医概念中分子之间的动态相互作用获益更多。使用分离的血 管, 我可以确定麻黄汤中的其他草药降低了麻黄的副作用。此外, 从西方的角度来 看, 药方中几种中草药的动态作用在受体结合实验的结果也是出乎意料的, 甚至有 悖常理。这也从西方的角度证实了这一动态的相互作用, 为研究中医的相互作用提 供了新的视角。

我们仍然需要扩展不同的 hard/softness 如何产生不同的氧化还原调节效果方面的 知识。在氧化还原调节网络中, 黄酮类化合物被氧化成黄酮类醌, 可通过抗坏血酸 再还原成黄酮类化合物, 并与谷胱甘肽形成谷胱甘肽加合物。表儿茶素是一种 soft 化合物, 与抗坏血酸的反应比谷胱甘肽快。然而, 作为相对 hard 化合物, 䚞皮素和 
monoHER 优先与抗坏血酸而不是谷胱甘肽反应。氧化还原调节化合物之间的这种差 异可能在保护我们的身体免受氧化损伤方面表现不同，应进一步研究。

还可以研究其他形式的能量, 例如: 光。研究发现中药可以“纠正”身体传播的光。 这个结论还不能用西方科学来解释。东方药物和其他类型的传统药物中还有许多其 他神秘的“力量”缺乏“西方科学基础”, 因此未被使用甚至可能丢失。西方科学家应该 继续保持思想开放, 学习中医, 连接东西方。毫无疑问, 当东西方联系更加紧密时, 医学将会得到改善, 我们将获得两全其美的优势。 


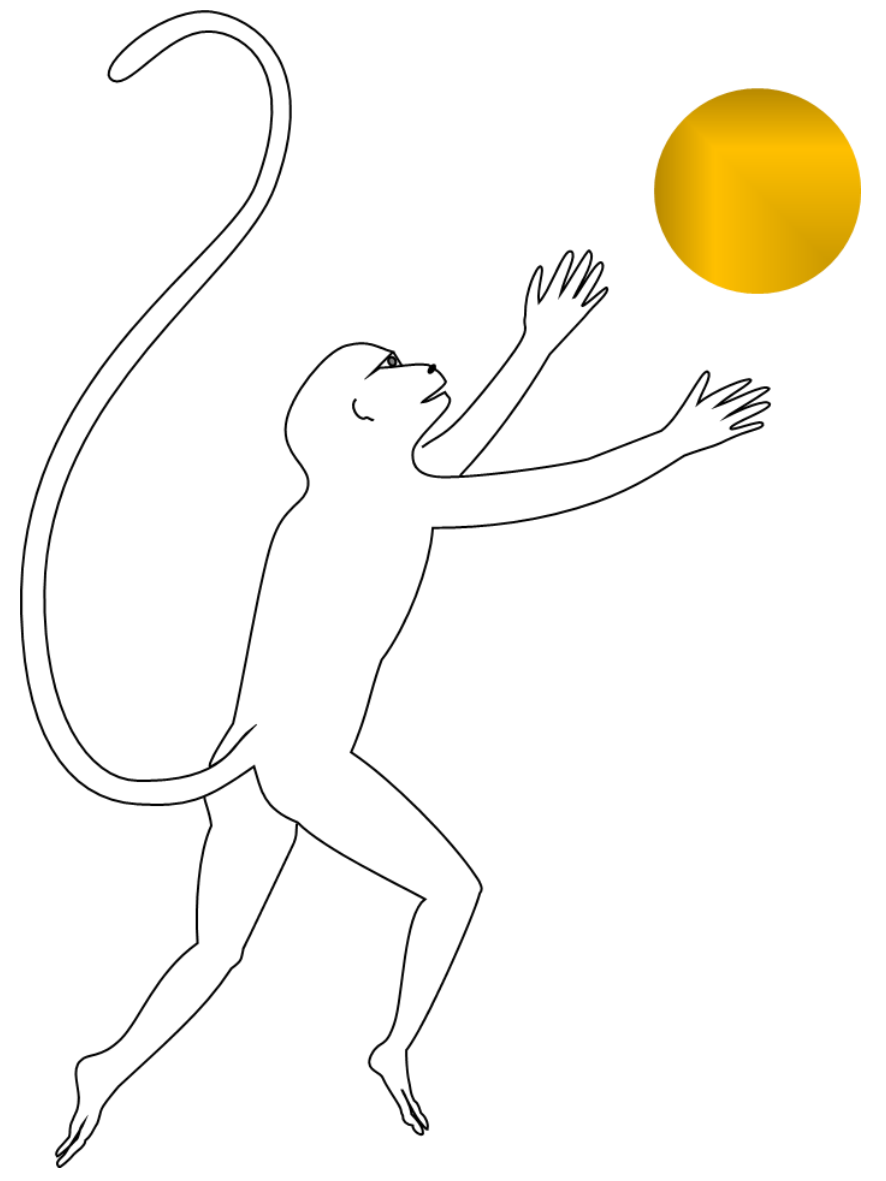



Acknowledgment 


\section{Acknowledgment}

This thesis carries my name. Actually, it is the concerted action of many people. I would like to thank everybody that directly and indirectly contributed and helped me to finish the thesis, and I am grateful to all the people I was allowed to cooperate with. This includes family and friends, teachers and students, colleagues and all the other people that gently and patiently polished me to produce the knowledge put in this thesis to become a philosophical doctor. I am pleased I can share this with you. To acknowledge and honor everybody, I would like to ask you - dear reader - to share, build on, and expend this knowledge.

上善若水。
水善利万物而不争,
与众人之所恶,
故几于道。
尽善也,
心善渊,
与善仁,
言善信,
政善治,
事善能,
动善时。
夫惟不争,
故无忧。

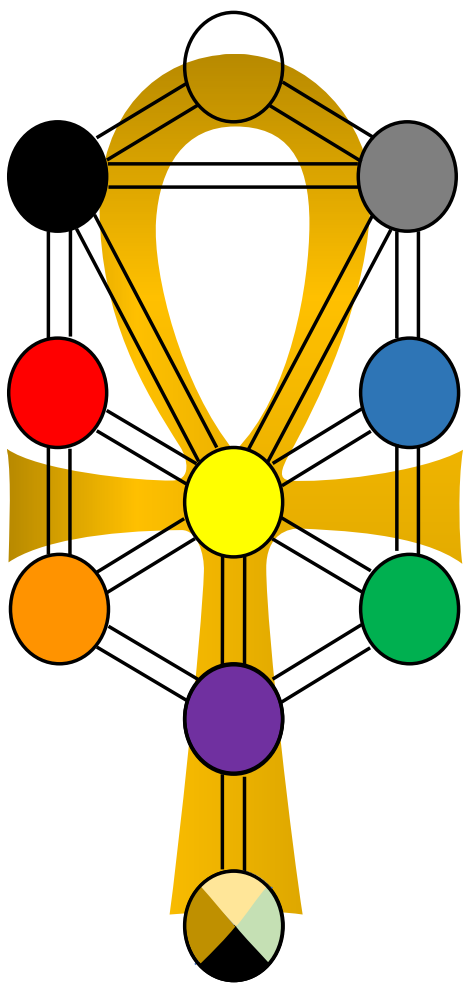

Het hoogste goed is als water.

Water geeft.

Onzichtbaar, onvoorwaardelijk en onbaatzuchtig. Onaantastbaar en onvergankelijk, en alles omvattend. Overal naar toe, niets wordt vergeten.

Daarom is het de oorsprong en wijst het ons de weg.

En zacht en weldadig blijft het stromen om te blijven geven. 




\section{List of publications}




\section{List of publications}

1. Zhang, M., Vrolijk, M., \& Haenen, G. (2017). The Screening of Anticholinergic Accumulation by Traditional Chinese Medicine. International journal of molecular sciences, 19(1), 18.

2. Zhang, M., Schiffers, P., Janssen, G., Vrolijk, M., Vangrieken, P., \& Haenen, G. R. (2018). The cardiovascular side effects of Ma Huang due to its use in isolation in the Western world. European Journal of Integrative Medicine, 18, 18-22.

3. Zhang, M., Vervoort, L., Moalin, M., Mommers, A., Douny, C., den Hartog, G. J., \& Haenen, G. R. (2018). The chemical reactivity of (-)-epicatechin quinone mainly resides in its B-ring. Free Radical Biology and Medicine, 124, 31-39.

4. Zhang, M., Moalin, M., Vervoort, L., Li, Z. W., Wu, W. B., \& Haenen, G. (2019). Connecting Western and Eastern Medicine from an Energy Perspective. International journal of molecular sciences, 20(6), 1512.

5. Zhang, M., Moalin, M., Mommers \& Haenen, G. R. (2019). Connecting West and East. International journal of molecular sciences, 20(9), 2333.

6. Zhang, M., Moalin, M., Li, Z. W., Chayoua, M. \& Haenen, G. Unravelling redox modulating activity of quercetin unraveled: a systematic approach. In preparation.
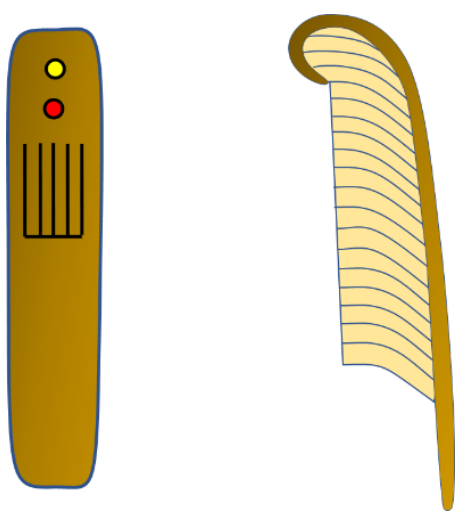




\section{Curriculum vitae}




\section{Curriculum vitae}

Ming Zhang (张明) was born on December 23th, 1988 in Henan Province, China. In September 2007, she started the Bachelor study Food Science and Engineering at Henan University of Technology. In September 2011, she began her master study in Food Engineering at Hainan University and conducted her internship at Coconut Research Institute of Chinese Academy of Tropical Agricultural Sciences. In October 2015, she started her PhD project at Department of Pharmacology and Toxicology, Maastricht University. The research performed is described in this thesis, under the supervision of prof. dr. Guido Haenen and dr. Mohamed

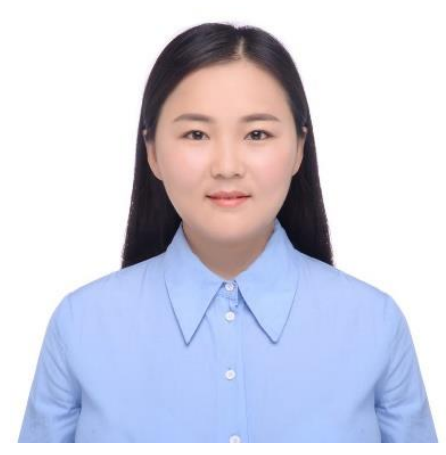
Moalin.

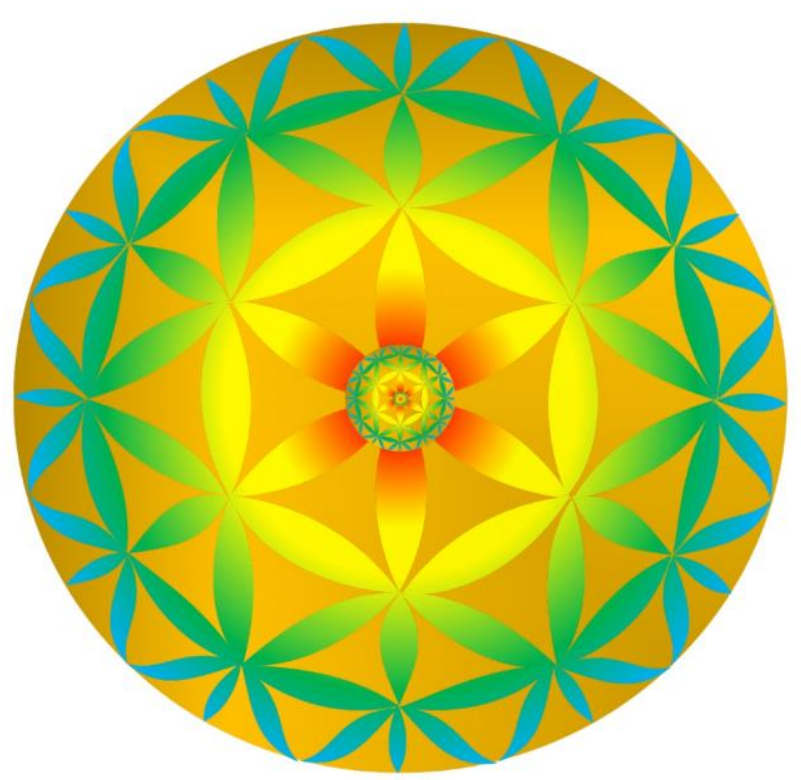





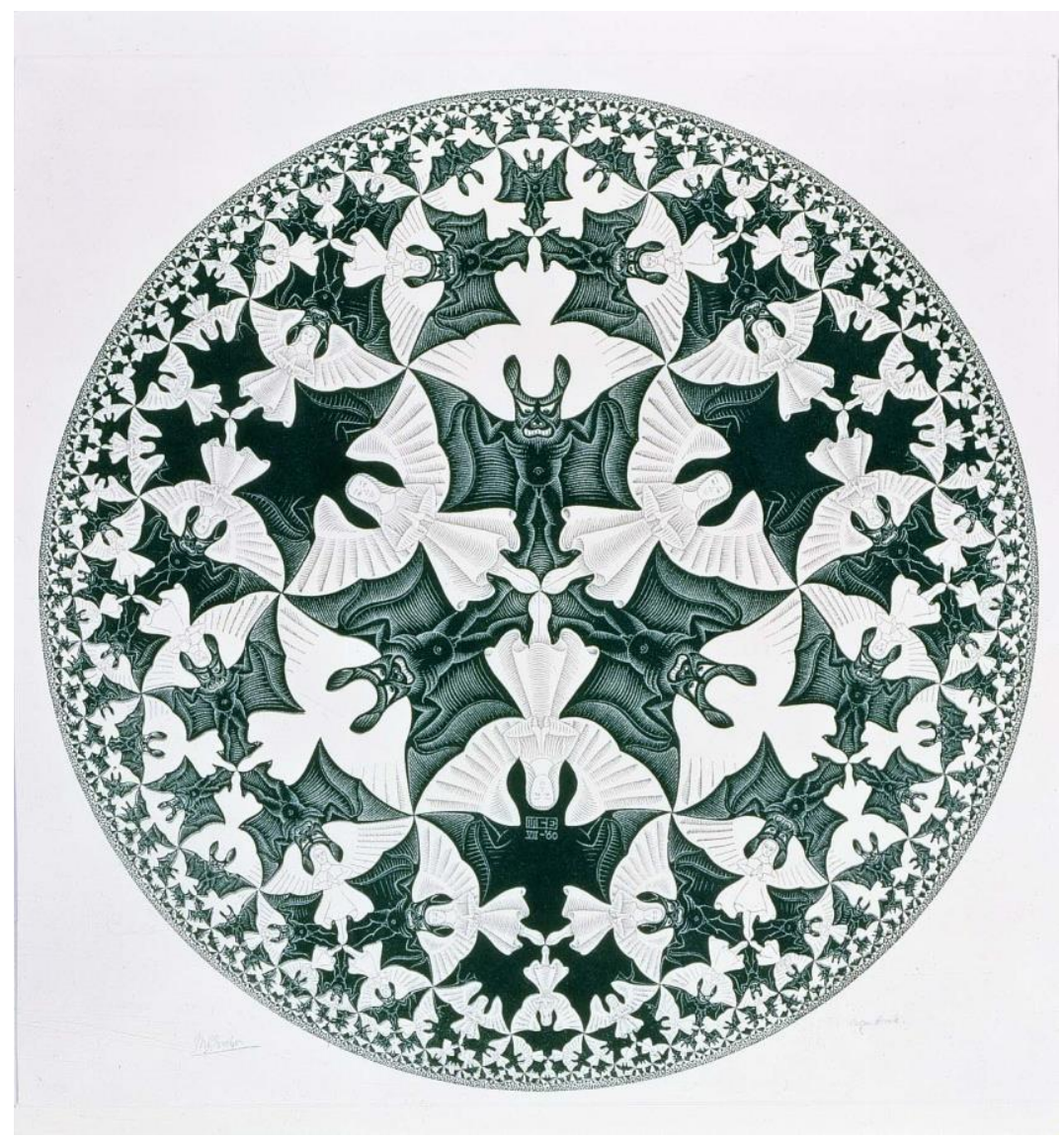

"Circle limit IV", by the Dutch master M.C. Escher, which can be considered as a Western vision of the Eastern Tai Ji symbol. This also depicts the unification of the two seemingly opposing worlds. This picture is used with the permission of the copyright owner, M.C. Escher's "Circle Limit IV" (C) 2019 The M.C. Escher Company-The Netherlands. All rights reserved. www.mcescher.com. 